\title{
Planck 2013 results. XXX. Cosmic infrared background measurements and implications for star formation
}

Planck Collaboration: P. A. R. Ade ${ }^{91}$, N. Aghanim ${ }^{64}$, C. Armitage-Caplan ${ }^{96}$, M. Arnaud ${ }^{77}$, M. Ashdown ${ }^{74,7}$, F. Atrio-Barandela ${ }^{20}$, J. Aumont ${ }^{64}$, C. Baccigalupi ${ }^{90}$, A. J. Banday ${ }^{99,11}$, R. B. Barreiro ${ }^{71}$, J. G. Bartlett ${ }^{1,72}$, E. Battaner ${ }^{101}$, K. Benabed ${ }^{65,98}$, A. Benoît ${ }^{62}$, A. Benoit-Lévy ${ }^{27,65,98}$, J.-P. Bernard ${ }^{99,11}$, M. Bersanelli ${ }^{38,54}$, M. Bethermin ${ }^{77}$, P. Bielewicz ${ }^{99,11,90}$, K. Blagrave ${ }^{10}$, J. Bobin ${ }^{77}$, J. J. Bock ${ }^{72,12}$, A. Bonaldi ${ }^{73}$, J. R. Bond ${ }^{10}$, J. Borrill ${ }^{15,93}$, F. R. Bouchet ${ }^{65,98}$, F. Boulanger ${ }^{64}$, M. Bridges ${ }^{74,7,68}$, M. Bucher ${ }^{1}$, C. Burigana ${ }^{53,36}$, R. C. Butler ${ }^{53}$, J.-F. Cardoso ${ }^{78,1,65}$,

A. Catalano ${ }^{79,76}$, A. Challinor ${ }^{68,74,13}$, A. Chamballu ${ }^{77,17,64}$, X. Chen ${ }^{61}$, H. C. Chiang ${ }^{30,8}$, L.-Y Chiang ${ }^{67}$, P. R. Christensen ${ }^{86,41}$, S. Church ${ }^{95}$, D. L. Clements ${ }^{60}$, S. Colombi ${ }^{65,98}$, L. P. L. Colombo ${ }^{26,72}$, F. Couchot ${ }^{75}$, A. Coulais ${ }^{76}$, B. P. Crill ${ }^{72,87}$, A. Curto ${ }^{7,71}$, F. Cuttaia $^{53}$, L. Danese $^{90}$, R. D. Davies ${ }^{73}$, R. J. Davis ${ }^{73}$, P. de Bernardis ${ }^{37}$, A. de Rosa ${ }^{53}$, G. de Zotti ${ }^{49,90}$, J. Delabrouille ${ }^{1}$, J.-M. Delouis ${ }^{65,98}$, F.-X. Désert ${ }^{57}$, C. Dickinson ${ }^{73}$, J. M. Diego ${ }^{71}$, H. Dole ${ }^{64,63}$, S. Donzelli ${ }^{54}$, O. Doré ${ }^{72,12}$, M. Douspis ${ }^{64}$, X. Dupac ${ }^{44}$, G. Efstathiou ${ }^{68}$, T. A. Enßlin ${ }^{82}$, H. K. Eriksen ${ }^{69}$, F. Finelli ${ }^{53,55}$,

O. Forni ${ }^{99,11}$, M. Frailis ${ }^{51}$, E. Franceschi ${ }^{53}$, S. Galeotta ${ }^{51}$, K. Ganga ${ }^{1}$, T. Ghosh ${ }^{64}$, M. Giard ${ }^{99,11}$, Y. Giraud-Héraud ${ }^{1}$, J. González-Nuevo ${ }^{71,90}$, K. M. Górski ${ }^{72,102}$, S. Gratton ${ }^{74,68}$, A. Gregorio ${ }^{39,51}$, A. Gruppuso ${ }^{53}$, F. K. Hansen ${ }^{69}$, D. Hanson ${ }^{83,72,10}$, D. Harrison ${ }^{68,74}$, G. Helou $^{12}$, S. Henrot-Versillé ${ }^{75}$, C. Hernández-Monteagudo ${ }^{14,82}$, D. Herranz ${ }^{71}$, S. R. Hildebrandt ${ }^{12}$, E. Hivon ${ }^{65,98}$, M. Hobson ${ }^{7}$, W. A. Holmes ${ }^{72}$, A. Hornstrup ${ }^{18}$, W. Hovest ${ }^{82}$, K. M. Huffenberger ${ }^{28}$, A. H. Jaffe ${ }^{60}$, T. R. Jaffe ${ }^{99,11}$, W. C. Jones ${ }^{30}$, M. Juvela ${ }^{29}$, P. Kalberla ${ }^{6}$, E. Keihänen ${ }^{29}$, J. Kerp ${ }^{6}$, R. Keskitalo ${ }^{24,15}$, T. S. Kisner ${ }^{81}$, R. Kneissl ${ }^{43,9}$, J. Knoche ${ }^{82}$, L. Knox ${ }^{32}$, M. Kunz ${ }^{19,64,3}$, H. Kurki-Suonio ${ }^{29,48}$, F. Lacasa ${ }^{64}$,

G. Lagache ${ }^{64, \star}$, A. Lähteenmäki ${ }^{2,48}$, J.-M. Lamarre ${ }^{76}$, M. Langer ${ }^{64}$, A. Lasenby ${ }^{7,74}$, R. J. Laureijs ${ }^{45}$, C. R. Lawrence ${ }^{72}$, R. Leonardi ${ }^{44}$, J. León-Tavares ${ }^{46,2}$, J. Lesgourgues ${ }^{97,89}$, M. Liguori ${ }^{35}$, P. B. Lilje ${ }^{69}$, M. Linden-Vørnle ${ }^{18}$, M. López-Caniego ${ }^{71}$, P. M. Lubin ${ }^{33}$,

J. F. Macías-Pérez ${ }^{79}$, B. Maffei ${ }^{73}$, D. Maino ${ }^{38,54}$, N. Mandolesi ${ }^{53,5,36}$, M. Maris ${ }^{51}$, D. J. Marshall ${ }^{77}$, P. G. Martin ${ }^{10}$, E. Martínez-González ${ }^{71}$,

S. Masi ${ }^{37}$, M. Massardi ${ }^{52}$, S. Matarrese ${ }^{35}$, F. Matthai ${ }^{82}$, P. Mazzotta $^{40}$, A. Melchiorri ${ }^{37,56}$, L. Mendes ${ }^{44}$, A. Mennella ${ }^{38,54}$, M. Migliaccio $^{68,74}$, S. Mitra ${ }^{59,72}$, M.-A. Miville-Deschênes ${ }^{64,10}$, A. Moneti ${ }^{65}$, L. Montier ${ }^{99,11}$, G. Morgante ${ }^{53}$, D. Mortlock ${ }^{60}$, D. Munshi ${ }^{91}$, J. A. Murphy ${ }^{85}$, P. Naselsky ${ }^{86,41}$, F. Nati ${ }^{37}$, P. Natoli ${ }^{36,4,53}$, C. B. Netterfield ${ }^{22}$, H. U. Nørgaard-Nielsen ${ }^{18}$, F. Noviello ${ }^{73}$, D. Novikov ${ }^{60}$, I. Novikov ${ }^{86}$, S. Osborne $^{95}$, C. A. Oxborrow ${ }^{18}$, F. Paci ${ }^{90}$, L. Pagano ${ }^{37,56}$, F. Pajot ${ }^{64}$, R. Paladini ${ }^{61}$, D. Paoletti ${ }^{53,55}$, B. Partridge ${ }^{47}$, F. Pasian ${ }^{51}$, G. Patanchon ${ }^{1}$, O. Perdereau ${ }^{75}$, L. Perotto $^{79}$, F. Perrotta ${ }^{90}$, F. Piacentini ${ }^{37}$, M. Piat ${ }^{1}$, E. Pierpaoli ${ }^{26}$, D. Pietrobon ${ }^{72}$, S. Plaszczynski ${ }^{75}$, E. Pointecouteau ${ }^{99,11}$, G. Polenta $^{4,50}$, N. Ponthieu ${ }^{64,57}$, L. Popa ${ }^{66}$, T. Poutanen ${ }^{48,29,2}$, G. W. Pratt ${ }^{77}$, G. Prézeau ${ }^{12,72}$, S. Prunet ${ }^{65,98}$, J.-L. Puget ${ }^{64}$, J. P. Rachen ${ }^{23,82}$, W. T. Reach ${ }^{100}$, R. Rebolo ${ }^{70,16,42}$, M. Reinecke ${ }^{82}$, M. Remazeilles ${ }^{73,64,1}$, C. Renault ${ }^{79}$, S. Ricciardi $^{53}$, T. Riller ${ }^{82}$, I. Ristorcelli ${ }^{99,11}$, G. Rocha ${ }^{72,12}$, C. Rosset ${ }^{1}$, G. Roudier ${ }^{1,76,72}$, M. Rowan-Robinson ${ }^{60}$, J. A. Rubiño-Martín ${ }^{70,42}$, B. Rusholme ${ }^{61}$, M. Sandri ${ }^{53}$, D. Santos ${ }^{79}$, G. Savini ${ }^{88}$, D. Scott ${ }^{25}$, M. D. Seiffert ${ }^{72,12}$, P. Serra ${ }^{64}$, E. P. S. Shellard ${ }^{13}$, L. D. Spencer ${ }^{91}$, J.-L. Starck ${ }^{77}$, V. Stolyarov ${ }^{7,74,94}$, R. Stompor ${ }^{1}$, R. Sudiwala ${ }^{91}$, R. Sunyaev $^{82,92}$, F. Sureau ${ }^{77}$, D. Sutton ${ }^{68,74}$, A.-S. Suur-Uski ${ }^{29,48}$, J.-F. Sygnet ${ }^{65}$, J. A. Tauber ${ }^{45}$, D. Tavagnacco ${ }^{51,39}$, L. Terenzi ${ }^{53}$, L. Toffolatti $^{21,71}$, M. Tomasi ${ }^{54}$, M. Tristram ${ }^{75}$, M. Tucci ${ }^{19,75}$, J. Tuovinen ${ }^{84}$, M. Türler ${ }^{58}$, L. Valenziano ${ }^{53}$, J. Valiviita ${ }^{48,29,69}$, B. Van Tent ${ }^{80}$, P. Vielva $^{71}$, F. Villa ${ }^{53}$, N. Vittorio $^{40}$, L. A. Wade ${ }^{72}$, B. D. Wandelt ${ }^{65,98,34}$, N. Welikala ${ }^{1}$, M. White ${ }^{31}$, S. D. M. White ${ }^{82}$, B. Winkel ${ }^{6}$, D. Yvon ${ }^{17}$, A. Zacchei ${ }^{51}$, and A. Zonca ${ }^{33}$

(Affiliations can be found after the references)

Received 17 June 2013 / Accepted 5 December 2013

\section{ABSTRACT}

\begin{abstract}
We present new measurements of cosmic infrared background (CIB) anisotropies using Planck. Combining HFI data with IRAS, the angular auto- and cross-frequency power spectrum is measured from 143 to $3000 \mathrm{GHz}$, and the auto-bispectrum from 217 to $545 \mathrm{GHz}$. The total areas used to compute the CIB power spectrum and bispectrum are about 2240 and $4400 \mathrm{deg}^{2}$, respectively. After careful removal of the contaminants (cosmic microwave background anisotropies, Galactic dust, and Sunyaev-Zeldovich emission), and a complete study of systematics, the CIB power spectrum is measured with unprecedented signal to noise ratio from angular multipoles $\ell \sim 150$ to 2500 . The bispectrum due to the clustering of dusty, star-forming galaxies is measured from $\ell \sim 130$ to 1100 , with a total signal to noise ratio of around 6, 19, and 29 at 217, 353, and 545 GHz, respectively. Two approaches are developed for modelling CIB power spectrum anisotropies. The first approach takes advantage of the unique measurements by Planck at large angular scales, and models only the linear part of the power spectrum, with a mean bias of dark matter haloes hosting dusty galaxies at a given redshift weighted by their contribution to the emissivities. The second approach is based on a model that associates star-forming galaxies with dark matter haloes and their subhaloes, using a parametrized relation between the dust-processed infrared luminosity and (sub-)halo mass. The two approaches simultaneously fit all auto- and cross-power spectra very well. We find that the star formation history is well constrained up to redshifts around 2, and agrees with recent estimates of the obscured star-formation density using Spitzer and Herschel. However, at higher redshift, the accuracy of the star formation history measurement is strongly degraded by the uncertainty in the spectral energy distribution of CIB galaxies. We also find that the mean halo mass which is most efficient at hosting star formation is $\log \left(M_{\mathrm{eff}} / M_{\odot}\right)=12.6$ and that CIB galaxies have warmer temperatures as redshift increases. The CIB bispectrum is steeper than that expected from the power spectrum, although well fitted by a power law; this gives some information about the contribution of massive haloes to the CIB bispectrum. Finally, we show that the same halo occupation distribution can fit all power spectra simultaneously. The precise measurements enabled by Planck pose new challenges for the modelling of CIB anisotropies, indicating the power of using CIB anisotropies to understand the process of galaxy formation.
\end{abstract}

Key words. cosmology: observations - large-scale structure of Universe - galaxies: star formation - infrared: diffuse background

^ Corresponding author: G. Lagache, e-mail: guilaine.lagache@ias.u-psud.fr 


\section{Introduction}

This paper, one of a set associated with the 2013 release of data from the Planck ${ }^{1}$ mission (Planck Collaboration I 2014), describes new measurements of the cosmic infrared background (CIB) anisotropy power spectrum and bispectrum, and their use in constraining the cosmic evolution of the star formation density and the luminous-dark matter bias.

The relic emission from galaxies formed throughout cosmic history appears as a diffuse, cosmological background. The CIB is the far-infrared part of this emission and it contains about half of its total energy (Dole et al. 2006). Produced by the stellarheated dust within galaxies, the CIB carries a wealth of information about the process of star formation. Because dusty, starforming galaxies at high redshift are extremely difficult to detect individually (e.g., Blain et al. 1998; Lagache et al. 2003; Dole et al. 2004; Fernandez-Conde et al. 2008; Nguyen et al. 2010), the CIB represents an exceptional tool for studying these objects and for tracing their overall distribution (Knox et al. 2001). The anisotropies detected in this background light trace the largescale distribution of star-forming galaxies and, to some extent, the underlying distribution of the dark matter haloes in which galaxies reside. The CIB is thus a direct probe of the interplay between baryons and dark matter throughout cosmic time.

The CIB has a redshift depth that complements current optical or near infrared measurements. This characteristic can be used to explore the early build-up and evolution of galaxies, one of the main frontiers in cosmology. Indeed the hope is to be able to use CIB anisotropies to improve our understanding of early gas accretion and star formation, and to assess the impact of galaxies on reionization. As dusty star-forming galaxies start to be found up to very high redshift (e.g., $z=6.34$, Riechers et al. 2013 ), this objective may be reachable, even if quantifying the $z \lesssim 6$ contribution to CIB anisotropy measurements to isolate the high-redshift part will be very challenging. However, as a start, CIB anisotropies can be used to measure the cosmic evolution of the star formation rate density (SFRD) up to $z \simeq 6$. Quantifying the SFRD at high redshift $(z>2.5)$ is a challenging endeavour. Currently, most of the measurements rely on the UV light emerging from the high-redshift galaxies themselves (e.g., Bouwens et al. 2009; Cucciati et al. 2012). To estimate their contribution to the total SFRD one needs to apply the proper conversion between the observed UV rest-frame luminosity and the ongoing SFR. This conversion factor depends on the physical properties of the stellar population (initial mass function, metallicities and ages) and on the amount of dust extinction, and is thus rather uncertain. Despite the significant amount of effort aimed at better understanding the UV-continuum slope distribution at high redshift, this remains one of the main limitation to SFRD measurements. The uncertainty on this conversion sometimes leads to significant revision of the SFRD (e.g., Behroozi et al. 2013b; Bouwens et al. 2012; Castellano et al. 2012). Remarkably, the estimates are now routinely made up to $z \sim 8$ (e.g., Oesch et al. 2012a), and have even been pushed up to $z \sim 10$ (Oesch et al. $2012 b)$. One of the key questions, is how to quantify the contribution of the dusty, star-forming galaxies to the SFRD at high redshift. Since it is mostly impossible to account for this contribution on the basis of optical/near-IR surveys, the best approach

\footnotetext{
Planck (http://www.esa.int/Planck) is a project of the European Space Agency (ESA) with instruments provided by two scientific consortia funded by ESA member states (in particular the lead countries France and Italy), with contributions from NASA (USA) and telescope reflectors provided by a collaboration between ESA and a scientific consortium led and funded by Denmark.
}

is to use the dusty galaxy luminosity function measurements. However, such measurements at high redshift are challenging with the current data, and this is where the CIB anisotropies, with their unmatched redshift depth, come into play. The SFRD from dusty, star-forming galaxies can be determined from their mean emissivity per comoving unit volume, as derived from CIB anisotropy modelling.

The way galaxies populate dark matter haloes is another ingredient that enters into the CIB anisotropy modelling. In particular, the galaxy bias - the relationship between the spatial distribution of galaxies and the underlying dark matter density field is a result of the varied physics of galaxy formation which can cause the spatial distribution of visible baryons to differ from that of dark matter. If galaxy formation is mainly determined by local physical processes (such as hydrodynamics), the galaxy bias is then approximately constant on large scales (Coles 1993), and the galaxy density fluctuations are thus proportional to those of the dark matter. The proportionality coefficient here is usually called as the linear bias factor, $b$. Its dependence on the luminosity, morphology, mass, and redshift of galaxies provides important clues to how galaxies are formed. However, the linear biasing parameter is at best a crude approximation, since the true bias is likely to be nontrivial, i.e., non-linear and scale dependent, especially at high redshift. At high redshift, the biasing becomes more pronounced, as predicted by theory (e.g., Kaiser 1986; Mo \& White 1996; Wechsler et al. 1998), and confirmed by the strong clustering of dusty star-forming galaxies (e.g., Steidel et al. 1998; Blain et al. 2004; Cooray et al. 2010). CIB anisotropies can be used to constrain the biasing scheme for dusty star-forming galaxies, which is crucial for understanding the process and history of galaxy formation.

However, measuring the CIB anisotropies is not easy. First, the instrument systematics, pipeline transfer function and beams have to be very well understood and measured. One can take advantage of recent experiments such as Hershel and Planck, for which diffuse emission is measured with better accuracy than their IRAS and Spitzer predecessors. Second, extracting the CIB requires a very accurate component separation. Galactic dust, CMB anisotropies, emission from galaxy clusters through the thermal Sunyaev Zeldovich (tSZ) effect, and point sources all have a part to play. In clean regions of the sky, Galactic dust dominates for multipoles $\lesssim 200$. This has a steep power spectrum (with a slope of about -2.8) and exhibits spatial temperature variations, and thus spectral energy distribution (SED) spatial variations. Distinguishing Galactic from extragalactic dust is very difficult, as their SEDs are quite similar, and both their spatial and spectral variations do not exhibit any particular features. Currently, the best approach is to rely on a Galactic template; taking another frequency is not recommended as CIB anisotropies also contribute. Taking a gas tracer as a spatial template is the best one can do, even if it has the drawback of not tracing the dust in all interstellar medium phases. For this purpose the CMB is very problematic at lower HFI frequencies, since its power spectrum is about 5000 and 500 times higher at $\ell=100$ than the CIB at 143 and $217 \mathrm{GHz}$, respectively. For Planck at $217 \mathrm{GHz}$ the $\mathrm{CMB}$ dominates the $\mathrm{CIB}$ for all $\ell$; at $353 \mathrm{GHz}$ it dominates for $\ell<1000$; and at $545 \mathrm{GHz}$ its power is 25 times lower at $\ell=100$ than the CIB. Any CMB template (taken from low-frequency data or from complex component separation algorithms) will be contaminated by residual foreground emission that will have to be corrected for. At Planck frequencies $v>200 \mathrm{GHz}$ the tSZ effect can be safely ignored, being close to zero at $217 \mathrm{GHz}$ and 100 times below the CIB power spectrum at $353 \mathrm{GHz}$. However tSZ contamination can 
Planck Collaboration: Planck 2013 results. XXX.

Table 1. Conversion factors, absolute calibration and inter-frequency relative calibration errors, beam FWHM and point source flux cuts, for HFI and IRIS.

\begin{tabular}{rcccccc}
\hline \hline $\begin{array}{c}\text { Band } \\
{[\mathrm{GHz}]}\end{array}$ & Map version & $\begin{array}{c}\text { Conversion factors } \\
\mathrm{MJy} \mathrm{sr}^{-1}\left[v I_{v}=\text { const.]/K }\right.\end{array}$ & $\begin{array}{c}\text { Abs. cal. error } \\
{[\%]}\end{array}$ & $\begin{array}{c}\text { Rel. cal. error } \\
{[\%]}\end{array}$ & $\begin{array}{c}F W H M \\
{[\operatorname{arcmin}]}\end{array}$ & $\begin{array}{c}\text { Flux cut } \\
{[\mathrm{mJy}]}\end{array}$ \\
\hline 100 & 2013 & $244.1 \pm 0.3$ & 0.54 & 0.3 & 9.66 & $400 \pm 50$ \\
143 & 2013 & $371.74 \pm 0.07$ & 0.54 & 0.3 & 7.27 & $350 \pm 50$ \\
217 & 2013 & $483.690 \pm 0.012$ & 0.54 & 0.3 & 5.01 & $225 \pm 50$ \\
353 & 2013 & $287.450 \pm 0.009$ & 1.24 & 1.0 & 4.86 & $315 \pm 50$ \\
545 & 2013 & $58.04 \pm 0.03$ & 10.0 & 5.0 & 4.84 & $350 \pm 50$ \\
857 & 2013 & $2.27 \pm 0.03$ & 10.0 & 5.0 & 4.63 & $710 \pm 50$ \\
3000 & IRIS & - & 13.5 & - & 4.30 & 1000 \\
\hline
\end{tabular}

Notes. Here "2013” means the first public release of Planck maps.

come from the use of a CMB template that contains residual tSZ power. At $100 \mathrm{GHz}$, based on the tSZ power spectrum measured in Planck Collaboration XXI (2014) and the CIB model developed in Planck Collaboration XVIII (2011), we estimate the tSZ power spectrum to be 10 times higher than the CIB. The correction of any tSZ contamination will be made difficult by the intrinsic correlation between the CIB and tSZ signals (Addison et al. 2012; Reichardt et al. 2012). Finally, as bright point sources will put extra power at all scales in the power spectrum, point sources need to be carefully masked up to a well-controlled flux density level. This step is complicated by the extragalactic source confusion that limits the depth of source detection for current far-infrared and submillimetre space missions.

The pioneering studies in CIB anisotropy measurement with Herschel-SPIRE (Amblard et al. 2011) and Planck-HFI (Planck Collaboration XVIII 2011) are now extended in Viero et al. (2013b) for the former, and in this paper for the latter. The new Planck measurements benefit from larger areas, lower instrument systematics, and better component separation. They are not limited to auto-power spectra but also include frequency crossspectra, from 143 to $857 \mathrm{GHz}$ (and $3000 \mathrm{GHz}$ with IRAS), and extend to bispectra at 217, 353, and $857 \mathrm{GHz}$. These more accurate measurements pose new challenges for the modelling of CIB anisotropies.

Our paper is organized as follows. We present in Sect. 2 the data we are using and the field selection. Section 3 is dedicated to the removal of the background $\mathrm{CMB}$ and foreground Galactic dust. We detail in Sect. 4 how we estimate the power spectrum and bispectrum of the residual maps, and their bias and errors. In the same section, results on CIB power spectra and bispectra are presented. In Sect. 5, we describe our modelling and show the constraints obtained on the SFRD, and the clustering of high-redshift, dusty galaxies. In Sect. 6, we discuss the $143 \mathrm{GHz}$ anisotropies, the frequency decoherence, the comparison of our measurements with previous determinations, the SFRD constraints, and the CIB non-Gaussianity. We conclude in Sect. 7. The appendices give some details about the HI data used to remove Galactic dust (Appendix A), the CIB anisotropy modelling (Appendices B and C), and also present the power spectra and bispectra tables (Appendix D).

Throughout the paper, we adopt the standard $\Lambda \mathrm{CDM}$ cosmological model as our fiducial background cosmology, with parameter values derived from the best-fit model of the CMB power spectrum measured by Planck (Planck Collaboration XVI 2014): $\left\{\Omega_{\mathrm{m}}, \Omega_{\Lambda}, \Omega_{\mathrm{b}} h^{2}, \sigma_{8}, h, n_{\mathrm{s}}\right\}=$ $\{0.3175,0.6825,0.022068,0.8344,0.6711,0.9624\}$. We also adopt a Salpeter initial mass function (IMF).

\section{Data sets and fields}

\subsection{Planck HFI data}

We used Planck channel maps from the six HFI frequencies: $100,143,217,353,545$, and $857 \mathrm{GHz}$. These are $N_{\text {side }}=2048$ HEALPix ${ }^{2}$ maps (Górski et al. 2005), corresponding to a pixel size of $1.72^{\prime}$. We made use of the first public release of HFI data that corresponds to temperature observations for the nominal Planck mission. The characteristics of the maps and how they were created are described in detail in the two HFI data processing and calibration papers (Planck Collaboration VI 2014; Planck Collaboration VIII 2014). At 857, 545, and $353 \mathrm{GHz}$, we use the zodiacal light subtracted maps (Planck Collaboration XIV 2014). Some relevant numbers for the CIB analysis are given in Table 1.

Maps are given in units either of $\mathrm{MJy} \mathrm{sr}^{-1}$ (with the photometric convention $v I_{v}=$ const. $)^{3}$ or $\mathrm{K}_{\mathrm{CMB}}$, the conversion between the two can be exactly computed knowing the bandpass filters. The mean coefficients used to convert frequency maps in $\mathrm{K}_{\mathrm{CMB}}$ units to $\mathrm{MJy} \mathrm{sr}^{-1}$ are computed using noise-weighted band-average spectral transmissions. The mean conversion factors are given in Table 1 . The map-making routines do not average individual detector maps, but instead combine individual detector data, weighted by the noise estimate, to produce singlefrequency channel maps. As portions of the sky are integrated for different times by different detectors, the relative contribution of a given detector to a channel-average map varies for different map pixels. The effects of this change on the channel-average transmission spectra is very small, being of the order of $0.05 \%$ for the nominal survey coverage (Planck Collaboration IX 2014; Planck Collaboration 2013).

As in Planck Collaboration XVIII (2011), the instrument noise power spectrum on the small extragalactic fields (see Sect. 2.3) is estimated with the jack-knife difference maps, which are built using the first and second halves of each pointing period (a half-pointing period is of the order of $20 \mathrm{~min}$ ). The half-ring maps give an estimate of the noise that is biased low (by a couple of percent) due to small correlations induced by the way the timelines have been deglitched (Planck Collaboration X 2014). However, as discussed in Planck Collaboration VI (2014), the bias is significant only at very high multipoles. As we stop our CIB power spectra measurements at $\ell=3000$, we can safely

\footnotetext{
2 http://healpix.sf.net

3 The convention $v I_{v}=$ const. means that the $\mathrm{MJy} \mathrm{sr}^{-1}$ are given for a source with a spectral energy distribution $I_{v} \propto v^{-1}$. For a source with a different spectral energy distribution a colour correction has to be applied (see Planck Collaboration IX 2014).
} 
A\&A 571, A30 (2014)

Table 2. CIB field description: centre (in Galactic coordinates), size, mean and dispersion of Hi column density.

\begin{tabular}{llrcccc}
\hline \hline Radio telescope & Field name & $\begin{array}{c}l \\
{[\mathrm{deg}]}\end{array}$ & $\begin{array}{c}b \\
{[\mathrm{deg}]}\end{array}$ & $\begin{array}{c}\text { Area } \\
{\left[\mathrm{deg}^{2}\right]}\end{array}$ & $\begin{array}{c}\text { Mean } N(\mathrm{HI}) \\
{\left[10^{20} \mathrm{~cm}^{-2}\right]}\end{array}$ & $\begin{array}{c}\sigma N(\mathrm{HI}) \\
{\left[10^{20} \mathrm{~cm}^{-2}\right]}\end{array}$ \\
\hline Effelsberg & EBHIS & 225 & 63 & 91.6 & 1.6 & 0.3 \\
\hline GBT & N1 & 85 & 44 & 26.4 & 1.2 & 0.3 \\
& AG & 165 & 66 & 26.4 & 1.8 & 0.6 \\
& SP & 132 & 48 & 26.4 & 1.2 & 0.3 \\
& LH2 & 152 & 53 & 16.2 & 0.7 & 0.2 \\
& Bootes & 58 & 69 & 54.6 & 1.1 & 0.2 \\
& NEP4 & 92 & 34 & 15.7 & 2.4 & 0.4 \\
& SPC5 & 132 & 31 & 24.6 & 2.3 & 0.6 \\
& SPC4 & 133 & 33 & 15.7 & 1.7 & 0.3 \\
& MC & 57 & -82 & 31.2 & 1.4 & 0.2 \\
\hline Parkes & GASS Mask1 & 225 & -64 & 1914 & 1.4 & 0.3 \\
& GASS Mask2 & 202 & -59 & 4397 & 2.0 & 0.8 \\
\hline
\end{tabular}

Notes. The given area is the size used for the CIB power spectrum computation (i.e., removing the area lost by the masking). The "GASS Mask2" is not used for CIB power spectrum analysis but for some tests on the quality of our component separation, and for the measurement of the bispectrum. Mask2 includes all of Mask1.

ignore this bias. For the larger GASS field (see Table 2), we directly compute the cross-spectra between the two half-maps to get rid of the noise (assuming that the noise is uncorrelated between the two half-maps).

The effective beam window functions, $b_{\ell}$, are determined from planet observations, folding in the Planck scanning strategy, as described in Planck Collaboration VII (2014). Because of the non-circular beam shape, the detector combinations, and the Planck scanning strategy, the effective $b_{\ell}(v)$ of each channel map applicable to the smallest patches considered here varies across the sky. These variations are, however, less than $1 \%$ at $\ell=2000$ and average out when considering many different patches, or larger sky areas. We will therefore ignore them and consider a single $b_{\ell}(v)$ for each frequency channel. Because of their experimental determination, the $b_{\ell}(v)$ are still affected by systematic uncertainties, which can be represented by a small set of orthogonal eigen-modes and whose relative standard deviation can reach $0.5 \%$ at $\ell=2000$ for $v=857 \mathrm{GHz}$. This uncertainty is accounted for in the error budget.

\subsection{IRIS data}

Our analysis uses far-infrared data at $3000 \mathrm{GHz}(100 \mu \mathrm{m})$ from IRAS (IRIS, Miville-Deschênes \& Lagache 2005). During its 10-month operation period, IRAS made two surveys of $98 \%$ of the sky and a third one of $75 \%$ of the sky. Each survey, called an HCON for Hours CONfirmation, consisted of two coverages of the sky separated by up to $36 \mathrm{~h}$. The first two HCONs (HCON-1 and HCON-2) were carried out concurrently, while the third survey (HCON-3) began after the first two were completed. Due to exhaustion of the liquid helium supply, the third HCON could not be completed. We use the HCON difference maps to estimate the instrument noise power spectrum (just as we use the half-pointing-period maps to estimate the instrument noise power spectrum for HFI). As for HFI at high frequencies, the IRIS $3000 \mathrm{GHz}$ map is given in $\mathrm{MJy} \mathrm{sr}^{-1}$ with the photometric convention $v I_{v}=$ const.

The beam at $3000 \mathrm{GHz}$ is not as well characterized as the HFI beams. The IRIS effective FWHM is about 4.3' (Miville-Deschênes \& Lagache 2005). We estimate the FWHM uncertainty using different measurements of the point spread function (PSF) coming from selected point sources used to study the PSF in the "ISSA explanatory supplement", and the power spectrum analysis from Miville-Deschênes et al. (2002). The dispersion between those estimates gives an uncertainty of $0.5^{\prime}$ on the FWHM.

\subsection{Extragalactic fields with high angular resolution $\mathrm{HI}$ data}

Following the successful approach of Planck Collaboration XVIII (2011), we do not remove Galactic dust by fitting for a power-law power spectrum at large angular scales, but rather use an independent, external tracer of diffuse dust emission, the HI gas. From $100 \mu \mathrm{m}$ to $1 \mathrm{~mm}$, at high Galactic latitude and outside molecular clouds a tight correlation is observed between far-infrared emission from dust and the 21-cm emission from gas $^{4}$ (e.g. Boulanger et al. 1996; Lagache et al. 1998; Planck Collaboration XXIV 2011). HI can thus be used as a tracer of cirrus emission in our fields, and indeed it is the best tracer of diffuse interstellar dust emission.

Although Planck is an all-sky survey, we restricted our CIB anisotropy measurements to a few fields at high Galactic latitude, where HI data at an angular resolution close to that of HFI are available. The 21-cm HI spectra used here were obtained with: (1) the Parkes 64-m telescope; (2) the Effelsberg 100-m radio telescope; and (3) the 100-m Green Bank telescope (GBT). Field characteristics are given in Table 2. Further details on the HI data reduction and field selection are given in Appendix A. The HEALPix HFI maps were reprojected onto the small HI GBT and EBHIS maps by binning the original HEALPix data into HI map pixels (Sanson-Flamsteed, or "SFL" projection with pixel size of $3.5^{\prime}$ for all fields). An average of slightly more than four HEALPix pixels were averaged for each small map pixel. For the GASS field, the HFI data were convolved to the HI angular resolution $\left(16.2^{\prime}\right)$, and then degraded to $N_{\text {side }}=512$.

We have 11 fields (one EBHIS, nine GBT and one GASS). The total area used to compute the CIB power spectrum is about $2240 \mathrm{deg}^{2}$, 16 times larger than in Planck Collaboration XVIII (2011).

\subsection{Point sources: flux cut and masks}

We use the Planck Catalogue of Compact Sources (PCCS, Planck Collaboration XXIX 2014) to identify point sources with

4 The Pearson correlation coefficient is $>0.9$ (Lagache et al. 2000). 
signal-to-noise ratio $(\mathrm{S} / \mathrm{N})$ greater or equal to 5 in the maps, and we create point-source masks at each frequency. We mask out a circular area of $3 \sigma$ radius around each source (where $\sigma=$ $F W H M / 2.35)$. The point sources to be removed have flux densities above a chosen threshold. The threshold is determined using the number counts $\mathrm{d} N / \mathrm{d} S$ on the cleanest $30 \%$ of the sky and by measuring the flux density at which we observe a departure from a Euclidean power law; this departure is a proxy for measuring the flux density regime where the incompleteness starts to be measurable. In practice, this departure often corresponds to about $80 \%$ of completeness (e.g., Planck Collaboration Int. VII 2013). The uncertainty on flux densities comes from the form of the $\mathrm{d} N / \mathrm{d} S$ slope. Flux density cuts and all-sky effective beam widths are given in Table 1 . For IRIS at $3000 \mathrm{GHz}$, we use the IRAS faint source catalogue (Moshir 1992) and we mask all sources with $S_{3000}>1 \mathrm{Jy}$.

\section{Extracting CIB from Planck-HFI and IRIS maps}

One of the most difficult steps in extracting the CIB is the removal of the Galactic dust and the CMB. CMB anisotropies contribute significantly to the total HFI map variance, in all channels at frequencies up to and including $353 \mathrm{GHz}$. Galactic dust contributes at all frequencies, and is dominant at the higher frequencies. One approach is to keep all the components and search for the best-fit model of the CIB in a likelihood approach, accounting for both $\mathrm{CMB}$ and dust (e.g., as a power law). This is the philosophy of the method developed to extract the cosmological parameters from the lower frequency data analysis (Planck Collaboration XV 2014; Planck Collaboration XVI 2014). However, with such an approach, the complexity of the likelihood, together with the number of parameters and their degeneracies prevent the use of advanced models for the clustered CIB (models beyond a simple power law). We thus decided to use another approach, based on template removal. To remove the $\mathrm{CMB}$ in the fields retained for our analysis, we used a simple subtraction technique (as in Planck Collaboration XVIII 2011). This method enables us to reliably evaluate CMB and foreground component residuals, as well as noise contamination, and to easily propagate errors (for example cross-calibration errors). It also guarantees that high-frequency CIB anisotropy signals will not leak into lower frequency, CMB-free maps. For Galactic dust, the present work focusses on very clean regions of the sky, for which Galactic foregrounds can be safely monitored using ancillary HI observations.

We describe in this section the removal of the two components. Some corrections are made at the map level, while others can only be done at the power spectrum level.

\section{1. $C M B$ removal}

\subsubsection{A low-frequency map as a CMB template}

The extraction of CIB anisotropies at low frequency is strongly limited by our ability to separate the CIB from the CMB. As a matter of fact, at multipole $\ell \sim 100$, the CIB anisotropy power spectrum represents about $0.2 \%$ and $0.04 \%$ of the CMB power spectrum at 217 and $143 \mathrm{GHz}$, respectively. We decided in this paper to use the HFI lowest frequency channel $(100 \mathrm{GHz})$ as a CMB template. This has the advantage of being an "internal" template, meaning its noise, data reduction processing, photometric calibration, and beam are all well known. It also has an angular resolution close to that of the higher frequency HFI channels. Following Planck Collaboration XVIII (2011), we removed the $\mathrm{CMB}$ contamination in the maps at $v \leq 353 \mathrm{GHz}$. At $545 \mathrm{GHz}$, because the CMB power represents less than $5 \%$ of the CIB power, we removed the $\mathrm{CMB}$ in harmonic space.

Using the lowest frequency channel as a CMB template ensures the lowest CIB contamination, since the CIB SED is decreasing as $v^{3.4}$ (Gispert et al. 2000). Contrary to what was done in Planck Collaboration XVIII (2011), where the $143 \mathrm{GHz}$ channel was used as a CMB template, we used the HFI $100 \mathrm{GHz}$ map here. This is a good compromise between being a low frequency template, and having an angular resolution close to the higher frequency HFI channels. Note that using the $100 \mathrm{GHz}$ channel was not feasible in Planck Collaboration XVIII (2011), because the data were too noisy, and the field area too small (140 versus $2240 \operatorname{deg}^{2}$ ).

As detailed in Planck Collaboration XVIII (2011), we applied a Wiener filter to the $100 \mathrm{GHz}$ map, designed to minimize the contamination of the CMB template by instrument noise. Errors in relative photometric calibration (between channels) are accounted for in the processing, as detailed in Sects. 4.1 and 4.2.

Following Planck Collaboration XVIII (2011), two corrections have to be applied to the measured CIB power spectra when using such a CMB template. First we need to remove the extra instrument noise that has been introduced by the CMB removal. This is done through:

$N_{\ell}^{\mathrm{CMBres}}(v)=N_{\ell}\left(v_{100}\right) \times w_{\ell}^{2} \times\left(\frac{b_{\ell}(v)}{b_{\ell}\left(v_{100}\right)}\right)^{2}$,

with $v$ equal to 143,217 or $353 \mathrm{GHz} . b_{\ell}(v)$ is the beam window function, $w_{\ell}$ is the Wiener filter, and $N_{\ell}\left(v_{100}\right)$ is the noise power spectrum of the $100 \mathrm{GHz}$ map. It is computed in the same way as in the other frequency channels, using the half-pointing period maps. Second, owing to the lower angular resolution of the $100 \mathrm{GHz}$ channel compared to 143 , and 217 and $353 \mathrm{GHz}$, we also have to remove the CMB contribution that is left close to the angular resolution of the 143, 217 and $353 \mathrm{GHz}$ channels:

$C_{\ell}^{\mathrm{CMBres}}(v)=C_{\ell}^{\mathrm{CMB}}(v) \times F_{\ell}^{2} \times b_{\ell}^{2}(v) \times\left(1-w_{\ell}\right)^{2}$,

with $F_{\ell}$ being the pixel and reprojection transfer function (detailed in Sect. 4.1).

Note that Eqs. (1) and (2) are the corrections for the autopower spectra. They are easily transposable to cross-power spectra.

\subsubsection{CMB template contamination to the CIB}

The low-frequency channel CMB template has the disadvantage of being contaminated by the CIB and the tSZ effect (the Galactic dust is removed using our dust model detailed in the following section, and IR and radio point sources are masked). Indeed the cross-spectra between the estimated CIB maps at $v$ and $v^{\prime}$ involve the $a_{\ell m}$ product

$$
\begin{aligned}
a_{\ell m}^{v} \times a_{\ell m}^{\nu^{\prime} *}= & \left\{a_{\ell m}^{\mathrm{CIB}, v}+a_{\ell m}^{\mathrm{SZ}, v}-w_{v}\left(a_{\ell m}^{\mathrm{CIB}, 100}+a_{\ell m}^{\mathrm{SZ}, 100}\right)\right\} \\
& \times\left\{a_{\ell m}^{\mathrm{CIB}, v^{\prime}}+a_{\ell m}^{\mathrm{SZ}, v^{\prime}}-w_{v^{\prime}}\left(a_{\ell m}^{\mathrm{CIB}, 100}+a_{\ell m}^{\mathrm{SZ}, 100}\right)\right\}^{*},
\end{aligned}
$$

where $w_{v}$ is either zero if $v \geq 545$, or the Wiener filter applied to the $100 \mathrm{GHz}$ map $\left(w_{\ell}\right)$ if $v \leq 353$.

Besides the signal $C_{\mathrm{CIB}}^{v \times v^{\prime}}$ that we want to measure, Eq. (3) involves three additional contributions that we have to correct for: $\mathrm{tSZ} \times \mathrm{tSZ}$; spurious $\mathrm{CIB} \times \mathrm{CIB}$; and $\mathrm{CIB} \times \mathrm{tSZ}$ correlations. We discuss each of them in this section. 


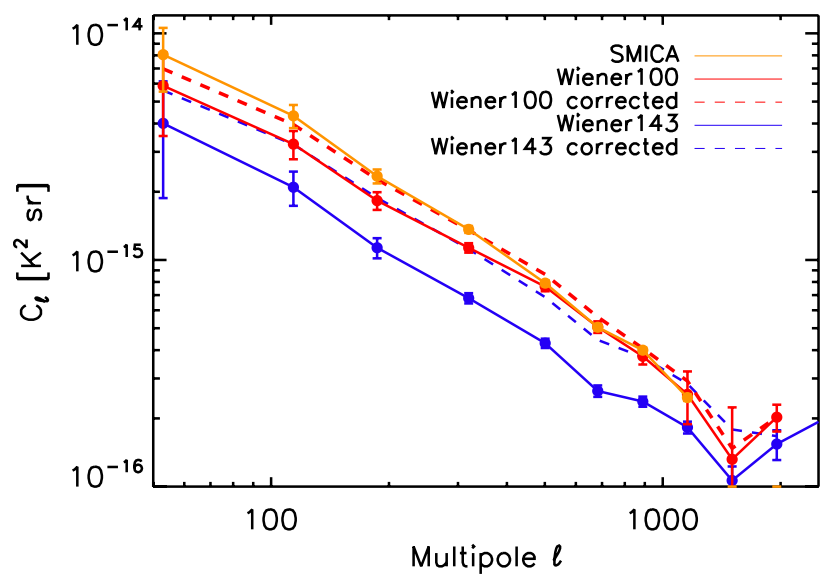

Fig. 1. Power spectrum of the residual map (map - dust - CMB) at $217 \mathrm{GHz}$ obtained in the GASS field with different CMB templates: the $100 \mathrm{GHz}$ Wiener-filtered map (red points); the $143 \mathrm{GHz}$ Wienerfiltered map (blue points); and the SMICA map (orange points). These CMB maps are contaminated by both CIB anisotropies and shot noise. For the CIB measured using Wiener-filtered CMB maps, we can easily compute the correction to apply to recover the true $\mathrm{CIB}$, using a model of the CIB anisotropies. Such corrected power spectra are shown with the dashed lines (using the CIB model from Planck Collaboration XVIII 2011). They are only indicative, since the correction strongly depends on the CIB model.

$\mathrm{CIB} \times \mathrm{CIB}$ spurious correlations - From Eq. (3), the $\mathrm{CIB} \times \mathrm{CIB}$ spurious contribution reads

$C_{\mathrm{CIBcorr}}^{\nu \times v^{\prime}}=-w_{\nu} C_{\mathrm{CIB}}^{100 \times v^{\prime}}-w_{\nu^{\prime}} C_{\mathrm{CIB}}^{100 \times v}+w_{\nu} w_{\nu^{\prime}} C_{\mathrm{CIB}}^{100 \times 100}$.

Using a model of CIB anisotropies, we can compute $C_{\text {CIBcorr }}^{\nu \times v^{\prime}}$. This correction includes both the shot noise and the clustered CIB anisotropies. The correction will be taken into account when searching for the best-fit CIB model: for each realization of our CIB anisotropy models (detailed in Sects. 5.4 and 5.5) we will compute $C_{\text {CIBcorr }}^{\nu \times v^{\prime}}$.

To illustrate the order of magnitude of the correction, we can use the model constructed in Planck Collaboration XVIII (2011) to fit the early Planck CIB measurements, and the shotnoise levels given in Sect. 5.1, and compute $C_{\mathrm{CIBcorr}}^{v \times v^{\prime}}$ when subtracting the $143 \mathrm{GHz}$ map instead of the $100 \mathrm{GHz}$ map as a $\mathrm{CMB}$ template. Note that this comparison is only indicative, since this CIB model at 143 and $100 \mathrm{GHz}$ is an extrapolation of the $217 \times 217$ power spectrum, using the clustering parameters of the $217 \mathrm{GHz}$ best-fit model, and the emissivities computed using the same empirical model of galaxy evolution. We show this comparison for the $217 \times 217$ power spectrum in Fig. 1. We see from this figure that the CIB obtained using the $143 \mathrm{GHz}$ map as a CMB template is about 1.6 times lower than the CIB obtained using the $100 \mathrm{GHz}$ map. Applying the correction (Eq. (4)) largely decreases this discrepancy (compare the two dashed lines ${ }^{5}$. Note that the correction is also nonnegligible when using the $100 \mathrm{GHz}$ map: it is a factor 1.15 for $50<\ell<700$. This justifies applying the correction systematically when fitting for our best CIB models.

We can extend the check on the impact of the choice of CMB map using the cross-correlation between the CIB and the distribution of dark matter, via the lensing effect on the CMB (Planck Collaboration XVIII 2014). We specifically compared

\footnotetext{
5 Note that the two curves do not have exactly the same level, as the corrections linked to the tSZ contributions are not the same when using the two CMB templates, and they are not applied for this plot.
}

the cross-correlation for the $\mathrm{CIB}$ at $217 \mathrm{GHz}$ obtained using our two Wiener-filtered 100 and $143 \mathrm{GHz}$ maps. We reached the same conclusion as before, and retrieved the same underestimate of the CIB when using the Wiener-filtered $143 \mathrm{GHz}$ rather than $100 \mathrm{GHz}$ CMB map.

Finally, we note that an alternative method of removing the CMB contamination was extensively tested; this was based on an internal linear combination of frequency maps, combined (or not) with a needlet analysis. However, such CMB maps are often not suited to our purposes because they are, among other problems, contaminated by the CIB that has leaked from the highfrequency channels that are used in the component separation process. We compare the CIB obtained using the $100 \mathrm{GHz}$ and SMICA CMB maps (Planck Collaboration XII 2014) in Fig. 1. We see that they are compatible within $1 \sigma$ (red-dashed line and orange points). Again this comparison is only indicative, since the SMICA CMB map main contaminants are SZ, shot noise from point sources, and $\mathrm{CIB}^{6}$; those contaminants have not been corrected for here, because the correction would need fully realistic simulations.

$\mathrm{tSZ} \times \mathrm{tSZ}$ correlation - From Eq. (3), the $\mathrm{tSZ} \times \mathrm{tSZ}$ spurious contribution reads

$C_{\mathrm{SZcorr}}^{\nu \times v^{\prime}}=C_{\mathrm{SZ}}^{\nu \times v^{\prime}}+w_{\nu} w_{\nu^{\prime}} C_{\mathrm{SZ}}^{100 \times 100}-w_{\nu^{\prime}} C_{\mathrm{SZ}}^{\nu \times 100}-w_{\nu} C_{\mathrm{SZ}}^{\nu^{\prime} \times 100}$.

We can make explicit the frequency dependence of the tSZ and write

$\Delta T_{\mathrm{SZ}}^{v}(\theta, \phi)=g_{v} \sum_{\ell m} a_{\ell m}^{\mathrm{SZ}}$

where $g_{v}$ is the conversion factor from the tSZ Compton parameter $y$ to $\mathrm{CMB}$ temperature units. Hence

$C_{\mathrm{SZ}}^{v \times v}=g_{v}^{2} C_{\mathrm{SZ}}$

Eq. (5) then becomes

$C_{\mathrm{SZcorr}}^{\nu \times v^{\prime}}=C_{\mathrm{SZ}}\left\{g_{\nu} g_{\nu^{\prime}}+w_{\nu} w_{\nu^{\prime}} g_{100}^{2}-g_{100}\left(w_{\nu^{\prime}} g_{\nu}+w_{\nu} g_{v^{\prime}}\right)\right\}$.

We compute $C_{\mathrm{SZcorr}}$ using the $\mathrm{tSZ}$ power spectrum, $C_{\mathrm{SZ}}$, and the conversion factors, $g_{v}$, given in Planck Collaboration XXI (2014). The uncertainty on $C_{\mathrm{SZ}}$ is about $10 \%$.

tSZ $\times$ CIB correlation - From Eq. (3), the $\mathrm{tSZ} \times \mathrm{CIB}$ spurious contribution reads

$$
\begin{aligned}
C_{\mathrm{CIB} \times \mathrm{SZ} \text { corr }}^{v \times v^{\prime}}= & C_{\mathrm{CIB} \times \mathrm{SZ}}^{v \times v^{\prime}}+C_{\mathrm{CIB} \times \mathrm{SZ}}^{\nu^{\prime} \times v}-w_{\nu} C_{\mathrm{CIB} \times \mathrm{SZ}}^{100 \times v^{\prime}}-w_{\nu^{\prime}} C_{\mathrm{CIB} \times \mathrm{SZ}}^{100 \times v} \\
& -w_{\nu} C_{\mathrm{CIB} \times \mathrm{SZ}}^{v^{\prime} \times 100}-w_{v^{\prime}} C_{\mathrm{CIB} \times \mathrm{SZ}}^{\nu \times 100}+2 w_{\nu} w_{\nu^{\prime}} C_{\mathrm{CIB} \times \mathrm{SZ}}^{100 \times 100}, \quad(9)
\end{aligned}
$$

where $C_{\mathrm{CIB} \times \mathrm{SZ}}^{\nu \times v^{\prime}}$ is the notation for the cross-spectrum between $\mathrm{CIB}(v)$ and $\mathrm{SZ}\left(v^{\prime}\right)$. This correction is highly dependent on the model used to compute the cross-correlation between tSZ and CIB; we use the model from Addison et al. (2012). We make the assumption that

$C_{\mathrm{CIB} \times \mathrm{SZ}}^{v \times v^{\prime}}=\left\langle a_{\ell m}^{\mathrm{CIB}}(v) a_{\ell m}^{\mathrm{SZ} *}\left(v^{\prime}\right)\right\rangle=g_{v^{\prime}} \phi(v) C_{\mathrm{CIB} \times \mathrm{SZ}}$,

where $\phi(v)$ is the amplitude of the power spectrum of the CIB correlated with the tSZ, $C_{\mathrm{CIB} \times \mathrm{SZ}}$, taken from Addison et al. (2012). This paper also provides examples of cross-spectra,

\footnotetext{
6 The contamination of the SMICA CMB map by foregrounds has been computed by running SMICA on the FFP6 simulations.
} 
Planck Collaboration: Planck 2013 results. XXX.

Table 3. SZ correction (Eq. (12)) to be applied to the CIB measurements at 217 and $353 \mathrm{GHz}$, in $\mathrm{Jy}^{2} \mathrm{sr}^{-1}\left[v I_{v}=\right.$ const.].

\begin{tabular}{rcccccc}
\hline \hline$\ell_{\text {cent }}$ & $C_{\text {SZcorr }}^{353 \times 333}$ & $C_{\text {CIB } \times \text { SZcorr }}^{353 \times 33}$ & $C_{\text {SZcorr }}^{217 \times 353}$ & $C_{\text {CIB } \times \text { SZcorr }}^{217 \times 353}$ & $C_{\text {SZcorr }}^{217 \times 217}$ & $C_{\text {CIB } \times \text { SZcorr }}^{217 \times 217}$ \\
\hline 53 & 643 & -395 & 446 & -170 & 309 & -46 \\
114 & 301 & -182 & 209 & -78 & 145 & -21 \\
187 & 184 & -110 & 128 & -47 & 89 & -13 \\
320 & 107 & -64 & 74 & -27 & 51 & -7.4 \\
501 & 66 & -40 & 46 & -17 & 31 & -4.7 \\
683 & 46 & -29 & 31 & -12 & 21 & -3.5 \\
890 & 32 & -22 & 21 & -9.2 & 14 & -2.6 \\
1157 & 18 & -15 & 9.2 & -5.6 & 4.7 & -1.7 \\
1504 & 7.5 & -9.4 & 1.3 & -2.2 & 0.2 & -0.5 \\
1956 & 4.4 & -6.7 & 0.2 & -1.2 & 0.0 & -0.1 \\
\hline
\end{tabular}

Notes. The uncertainty on $C_{\mathrm{SZcorr}}$ is of order $10 \%$, while the uncertainty on $C_{\mathrm{CIB} \times \mathrm{SZcorr}}$ is about a factor of two.

with a reference frequency at $150 \mathrm{GHz}$. We use this reference frequency and power spectrum ratios to compute $C_{\mathrm{SZ} \times \mathrm{CIB}}^{v \times v^{\prime}}$ following

$C_{\mathrm{SZ} \times \mathrm{CIB}}^{v \times v^{\prime}}=\frac{\phi\left(v^{\prime}\right)}{\phi(150)} \frac{g_{v}}{g_{150}} C_{\mathrm{SZ} \times \mathrm{CIB}}^{150 \times 150}$

We show in Fig. 2 the measured CIB, $C_{\mathrm{CIB}}^{\nu \times v^{\prime}}$ (measured), together with the corrected one:

$C_{\mathrm{CIB}}^{\nu \times v^{\prime}}=C_{\mathrm{CIB}}^{\nu \times v^{\prime}}$ (measured) $-\left\{\mathrm{C}_{\mathrm{SZcorr}}^{v \times v^{\prime}}+\mathrm{C}_{\mathrm{CIB} \times \mathrm{SZcorr}}^{\nu \times v^{\prime}}\right\}$.

We also show the ratio of the corrections to the measured CIB, and give the values of the corrections at 217 and $353 \mathrm{GHz}$ in Table 3. We see that tSZ contamination is the highest for the $217 \times 217$ combination, with a contamination of the order of $15 \%$. It is less than $5 \%$ and $1 \%$ for $217 \times 353$ and $353 \times$ 353 , respectively. The $\mathrm{tSZ}$ power spectrum measured by Planck Collaboration XXI (2014) is uncertain by $10 \%$, while tSZ $\times$ CIB is uncertain by a factor of two (Addison et al. 2012). Hopefully, where the contamination is important (i.e., $217 \times 217$ ), the dominant contribution to the CIB comes from $C_{\mathrm{SZ} \text { corr }}^{\nu \times v^{\prime}}$. Hereafter, we therefore apply the correction coming from the tSZ contamination to the measured CIB, and add the uncertainties of the correction quadratically to the $\mathrm{CIB}$ uncertainties.

When cross-correlating maps at $353 \mathrm{GHz}$ and above $545 \mathrm{GHz}$, the correction linked to the tSZ contamination is dominated by the term $C_{\mathrm{CIB} \times \mathrm{SZcorr}}^{v \times v^{\prime}}$, which is highly uncertain. The correction is about $3 \%,<1 \%, 4 \%$ and $<2 \%$ for the $217 \times 545$, $353 \times 545,217 \times 857$ and $353 \times 857$ cross-power spectra, respectively. Although small, for consistency with the case $v \leqslant 353 \mathrm{GHz}$ and $v^{\prime} \leqslant 353 \mathrm{GHz}$, we also apply the tSZrelated corrections to the measured CIB when $v \leqslant 353 \mathrm{GHz}$ and $v^{\prime} \geqslant 545 \mathrm{GHz}$.

\subsection{Dust model}

Many studies, using mostly IRAS and COBE data, have revealed the strong correlation between the far-infrared dust emission and 21-cm integrated emission at high Galactic latitudes. In particular, Boulanger et al. (1996) studied this relation over the whole high Galactic latitude sky and reported a tight dust-HI correlation for $N_{\mathrm{HI}}<4.6 \times 10^{20} \mathrm{~cm}^{-2}$. For higher column densities the dust emission systematically exceeds that expected by extrapolating the correlation. Examining specific high Galactic latitude regions, Arendt et al. (1998), Reach et al. (1998), Lagache et al. (1998 and 1999) found infrared excesses with respect to $N_{\mathrm{HI}}$, with a threshold varying from 1.5 to $5.0 \times 10^{20} \mathrm{~cm}^{-2}$.

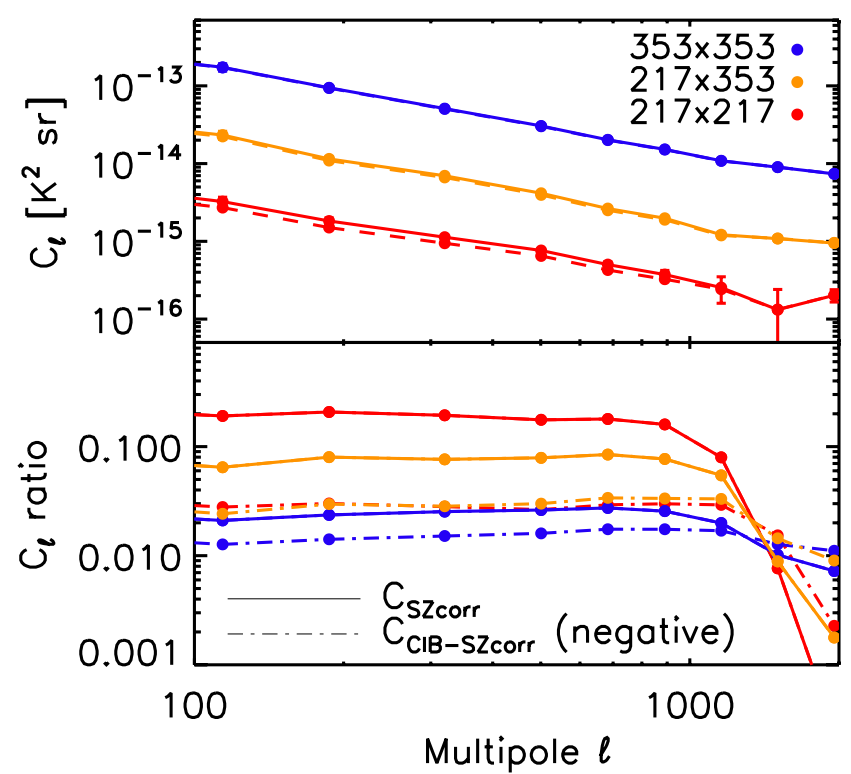

Fig. 2. Top: residual map (a.k.a CIB) auto- and cross-spectra measured at 217 and $353 \mathrm{GHz}$ (circles). The dashed lines represent the measured power spectra corrected for the tSZ contamination (both tSZ and tSZ $\times$ CIB, see Eqs. (8) and (9), respectively). Bottom: absolute value of the ratio $C_{\mathrm{SZcorr}}^{v \times v^{\prime}} / C_{\mathrm{CIB}}^{\nu \times v^{\prime}}$ [measured] (continuous line) and $C_{\mathrm{CIB}-\mathrm{SZcorr}}^{v \times v^{\prime}} / C_{\mathrm{CIB}}^{v \times v^{\prime}}$ [measured] (dot-dashed line, negative). The highest contamination is for the $217 \times 217$ power spectrum, where the total correction represents about $15 \%$ of the measured CIB power spectrum. For $C_{\mathrm{SZcorr}}^{\nu \times v^{\prime}}$, the uncertainty is about $10 \%$. On the contrary, $C_{\mathrm{ClB}-\mathrm{SZcorr}}^{\nu \times v^{\prime}}$ is poorly constrained, as the model used to compute it is uncertain by a factor of two.

Planck Collaboration XXIV (2011) presented the results from the comparison of Planck dust maps with GBT HI observations in 14 fields covering more than $800 \mathrm{deg}^{2}$. They showed that the brighter fields in their sample, with an average HI column density greater than about $2.5 \times 10^{20} \mathrm{~cm}^{-2}$, show significant excess dust emission compared to the HI column density. Regions of excess lie in organized structures that suggest the presence of hydrogen in molecular form. Because of this, we restrict our CIB analysis to the cleanest part of the sky, with mean $N_{\mathrm{HI}}<2.5 \times 10^{20} \mathrm{~cm}^{-2}$.

\subsubsection{Constructing dust maps}

As detailed in Planck Collaboration XXIV (2011), Planck Collaboration XVIII (2011) and in Appendix A, we constructed 
integrated HI emission maps of the different HI velocity components observed in each individual field: the local component, typical of high-latitude HI emission, intermediate-velocity clouds (IVCs), and high-velocity clouds (HVCs), if present. To remove the cirrus contamination from HFI maps, we determined the far-IR to millimetre emission of the different HI components. We assumed that the HFI maps, $I_{v}(x, y)$, at frequency $v$ can be represented by the following model

$I_{\nu}(x, y)=\sum_{i} \alpha_{v}^{i} N_{\mathrm{HI}}^{i}(x, y)+C_{v}(x, y)$

where $N_{\mathrm{HI}}^{i}(x, y)$ is the column density of the $i$ th HI component, $\alpha_{\gamma}^{i}$ is the far-IR to mm versus HI correlation coefficient of component $i$ at frequency $v$ and $C_{v}(x, y)$ is an offset. The correlation coefficients $\alpha_{v}^{i}$ (often called emissivities) were estimated using $\chi^{2}$ minimization given the HI, HFI and IRIS data, as well as the model (Eq. (13)). We removed from the HFI and IRIS maps the HI velocity maps multiplied by the correlation coefficients. For EBHIS and GBT fields, we only considered one correlation coefficient per field and per frequency. The removal was done at the HFI and IRIS angular resolutions, even though the HI map is of lower resolution $\left(\sim 10^{\prime}\right)$. This is not a problem because cirrus, with a roughly $k^{-2.8}$ power-law power spectrum (Miville-Deschênes et al. 2007), has negligible power between the HI (GBT and EBHIS) and HFI and IRIS angular resolutions, in comparison to the power in the CIB. The correlation of the dust emission with the different HI velocity components and its variation from field to field is illustrated in Fig. 5 of Planck Collaboration XVIII (2011).

For the GASS field, due to its large size the dust model needs to take into account variations of the dust emissivity across the field. We make use of an analysis of the dust-to-gas correlation over the southern Galactic cap $\left(b<-30^{\circ}\right)$ using HI data from the GASS southern sky survey (Kalberla et al. 2010). The Planck-HFI maps are linearly correlated with HI column density over an area of $7500 \mathrm{deg}^{2}$ covering all of the southern sky $\left(\delta<0^{\circ}\right)$ at $b<-30^{\circ}(17 \%$ of the sky). We use HFI maps corrected for the mean value of the CIB (Planck Collaboration VIII 2014). The Planck maps and HI emission at Galactic velocities are correlated over circular patches with $15^{\circ}$ diameters, centred on a HEALPix grid with Nside $=32$. The linear regression is iterated to identify and mask sky pixels that depart from the correlation. At microwave frequencies the correlation coefficients $\left(\alpha_{v}\right)$ and offsets $\left(C_{v}\right)$ derived from this linear correlation analysis include a significant $\mathrm{CMB}$ contribution that comes from the chance correlation of the cosmic background with the HI emission. This contribution is estimated by assuming that the SED of dust emission follows a modified blackbody spectrum for $100 \leq v \leq 353 \mathrm{GHz}$. The fit is performed on the differences $\alpha_{v}-\alpha_{100 \mathrm{GHz}}$ that are CMB-free for each sky area when expressed in units of $\mathrm{K}_{\mathrm{CMB}}$. This yields values of the correlations coefficients corrected for $\mathrm{CMB}, \alpha_{v}^{\mathrm{c}}$. The detailed procedure is described in Planck Collaboration (2013). In this section we explain how the results of this study are used to build a model of the dust contribution to the sky emission.

To make the dust model in the GASS field, we start by building a map of the dust emission to HI column density ratio, interpolating the values of $\alpha_{v}^{\mathrm{c}}$, corrected for the CMB, using HEALPix sky pixels with a Gaussian kernel. The $1 \sigma$ width of this convolution kernel is equal to the pixel size $1.8^{\circ}$ of the HEALPix grid for Nside $=32$. To reduce the data noise at $v<353 \mathrm{GHz}$ we use the modified blackbody fits to $\alpha_{v}-\alpha_{100 \mathrm{GHz}}$ and not the measured values of $\alpha_{v}$. This yields a set of six maps of the dust emission per unit HI column density for all HFI frequencies from 100 to $857 \mathrm{GHz}$. We also build a set of Galactic offset maps from the offsets $C_{v}$ of the Planck-HI correlation. These offsets comprise contributions from Galactic dust and the CMB. We subtract the CMB contribution assuming that the SED of the dust contribution to $C_{v}$ is the same as that of $\alpha_{v}^{\mathrm{c}}$ for each sky area. For each frequency, the dust model is the product of the dust emission per unit HI column density times the HI map, plus the Galactic offset map. The angular resolution of the model is that of the HI map $\left(16.2^{\prime}\right)$.

As for the smaller EBHIS and GBT fields, the model in the GASS field only accounts for the emission of dust in HI gas. Clouds with a significant fraction of molecular gas produce localized regions with positive residual emission. The histogram of residual emission at $857 \mathrm{GHz}$ also shows a non-Gaussian extension towards negative values (as observed in the EBHIS field, see Appendix A.3). In the maps these pixels correspond to localized HI clouds with no (or a weak) counterpart in the Planck map. In the GASS survey, these clouds are likely to be part of the Magellanic stream, with radial velocities within the range used to build the Galactic HI map. For the analysis of the CIB we mask pixels with positive and negative residuals larger than $3 \sigma$. To be conservative this first mask is slightly enlarged, and apodized. It covers about $4400 \mathrm{deg}^{2}$ (Mask2 in Table 2). This mask still contains some regions with $N_{\mathrm{HI}} \geq$ $2.5 \times 10^{20} \mathrm{~cm}^{-2}$ and thus potential IR emission from molecular gas clouds. To measure the CIB power spectrum, all regions with $N_{\mathrm{HI}} \geq 2.1 \times 10^{20} \mathrm{~cm}^{-2}$ are further masked (Mask1). The final area is about $1900 \mathrm{deg}^{2}$.

\subsubsection{Dust map uncertainties}

The uncertainties estimated for the emissivities by the leastsquares fit method are substantially underestimated, as they do not take into account systematic effects associated with the CIB and the CMB, nor the spatial variation of the emissivity inside a patch (or a field). We use the GASS field to estimate the error we have on the dust model. For this field, we have the largescale variations (at $\sim 15^{\circ}$ ) of the dust emissivities. We make the hypothesis that the measured variations extend to smaller angular scales, with a distribution on the sky given by a power spectrum with a slope -2.8 , similar to that of the dust emission. Within this assumption, we simulate multiple maps of the dust emissivity that all match the dispersion of the dust emissivity measured at $857 \mathrm{GHz}$. For each realization, we obtain a dust map at $857 \mathrm{GHz}$ by multiplying the dust emissivity map by the GASS map of Galactic HI. Dust maps at other frequencies are obtained by scaling the $857 \mathrm{GHz}$ flux using the mean dust SED given.These simulations provide a good match to the Galactic residuals of the dust-HI correlation characterized.We obtain simulated maps of the sky emission adding realizations of the HFI instrument noise, CIB and CMB to the dust maps. We perform on the simulated sky maps the same correlation analysis with HI as that done on the Planck maps. For each simulation, we obtain values of the dust emissivity that we compare with the input emissivity map averaged over each sky patch. We find that there is no systematic difference between the values derived from the correlation analysis and the input values of the dust emissivities. The fractional error, i.e., the standard deviation of the difference between measured and the input values divided by the mean dust emissivity, is $13 \%$ of the mean dust emissivity at $857 \mathrm{GHz}$. This error increases slightly towards lower frequencies up to 16 and $21 \%$ for the 143 and $100 \mathrm{GHz}$ channels. 


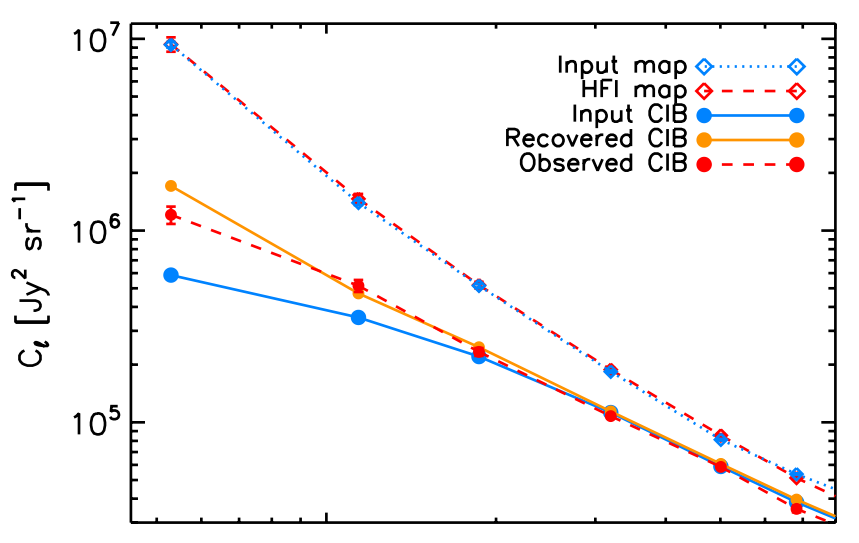

100

\section{Multipole $\ell$}

Fig. 3. Power spectrum analysis of the simulations made at $857 \mathrm{GHz}$ in the GASS field to characterize cirrus residuals. The blue and red diamonds compare the power spectrum of our simulated and HFI maps, respectively, while the blue and orange dots are the simulated and recovered CIB, respectively. The recovered CIB is biased by cirrus residuals at low multipoles. The measured CIB (obtained on the GASS Mask1 field, displayed with all but cirrus error bars) shows the same behaviour at low multipoles. Thus the measurements in the first two $\ell$ bins have to be considered as upper limits. As discussed in Sect. 3.2.2, the simulations are used to compute the error bars linked to the cirrus removal.

We show in Fig. 3 the power spectrum of the recovered CIB, compared to the input CIB. We see that it is strongly biased by Galactic dust residuals in the first two multipole bins. These points have thus to be considered as upper limits. For the other points, we use the simulations to set the error bars linked to the Galactic dust removal. The observed linear correlation between the sigmas of dust residuals and $N_{\mathrm{HI}}$ is used to compute the power spectrum of Galactic dust residuals for each field, following

$$
C_{\ell}^{\text {Field }}=C_{\ell}^{\mathrm{GASS}} \times\left(\frac{\left\langle N_{\mathrm{HI}}^{\text {Field }}\right\rangle}{\left\langle N_{\mathrm{HI}}^{\mathrm{GASS}}\right\rangle}\right)^{2} .
$$

\subsubsection{Galactic dust residuals}

One of the main issues when using HI column density as a dust tracer is the presence of emissivity variation on scales smaller that those probed by the correlation analysis, "dark gas" (see Planck Collaboration XIX 2011; Planck Collaboration XXIV 2011), and ionized gas (Lagache et al. 2000). The dust contribution of the dark gas becomes rapidly visible (not only at high frequencies), when $N_{\mathrm{HI}} \gtrsim 2.5 \times 10^{20} \mathrm{~cm}^{-2}$. In addition to the simulations discussed in the previous section, we investigated the contribution of dust residuals by computing the CIB power spectra on the GASS field, using Mask1 and Mask2. Mask1 is very conservative and is our nominal mask for CIB analysis. Mask 2 contains higher column density regions, so it is not suitable for CIB-only analysis, but is useful, for example, when cross-correlating the CIB with other large-scale structure tracers (when dust contamination is less of a problem, e.g., Planck Collaboration XVIII 2014) and for the bispectrum measurement at low frequencies. As expected, the power spectra of residual maps computed using Mask2 have an excess compared to the CIB. Whatever the frequency, this excess represents about 5$10 \%$ of the cirrus power spectrum at low $\ell$. This leads to a CIB from Mask2 that is overestimated by a factor of 1.5 at $857 \mathrm{GHz}$ and 1.2 at $217 \mathrm{GHz}$, for $\ell \leq 200$, compared to the CIB from Mask1. Taking such a residual at the level of 5-10\% of the dust power spectrum (as observed between Mask1 and Mask2) would strongly affect only the first two bins, at $\ell=53$ and 114 . This is consistent with the simulation analysis (see Fig. 3). We will thus not use those bins when searching for the best CIB model, and we consider them only as upper limits.

\subsection{CMB and Galactic dust cleaned maps}

We show in Fig. 4, one example of the cleaning process, from the frequency to the CIB maps, for the SPC5 field. The bottom row shows the residual CIB maps, smoothed to $10^{\prime}$. In Fig. 5 are shown the CIB maps in a part of the GASS field, at 16.2' resolution. We see from both figures that common structures, corresponding to CIB anisotropies, are clearly visible. As previously noticed (Planck Collaboration XVIII 2011), the three intermediate frequencies $(353,545$ and $857 \mathrm{GHz})$ show highly correlated structures. On the contrary, the $3000 \mathrm{GHz}$ data on one hand, and the $217 \mathrm{GHz}$ on the other, reveal a decoherence, which can be attributed to the redshift distribution of the CIB anisotropies. We will come back to this decoherence by measuring the correlation coefficients in Sect. 6.2.

There are two frequencies where extracting the CIB from the frequency maps is particularly challenging.

- At $3000 \mathrm{GHz}$, the cirrus contamination is the highest and we observe more spatial variations of the dust-HI emissivity which are difficult to manage. This is due to the contamination at $3000 \mathrm{GHz}$ data by a hotter dust component, which may be linked to the so-called "very small grains" in some interstellar filaments. Consequently, we have not tried to extract the CIB in the GASS field at $3000 \mathrm{GHz}$, and the Bootes field has been discarded for the CIB analysis due to interstellar dust residuals (due to one IVC component, which has both a high emissivity and a hot spectrum; P. Martin, priv. comm.). Moreover, the EBHIS field is right in the middle of the missing IRAS observation. It is thus also not used for the $\mathrm{CIB}$ analysis. In the end, the total area used to compute the CIB power spectrum at $3000 \mathrm{GHz}$ is $183 \mathrm{deg}^{2}$.

- At $143 \mathrm{GHz}$, we expect the correlated CIB anisotropies in brightness to be about $1-2 \%$ of the CMB anisotropies for multipoles $\ell \sim 100-1000$ (while it is about $3-15 \%$ at $217 \mathrm{GHz}$ ). The removal of the CMB has thus to be extremely accurate. Moreover, the expected CIB is lower than the instrument noise. Constraints can only be obtained on the large GASS field that is more immune to noise. We see from Fig. 5 that the $143 \mathrm{GHz}$ CIB map shows some structures that are correlated with the higher CIB frequency maps. We discuss in Sect. 6.1 our attempt to obtain some constraints at this frequency.

We used two different approaches to measure the CIB power spectra according to the size of the fields: (i) for the EBHIS and GBT flat-sky fields, we used an updated version of POKER (Ponthieu et al. 2011), and we computed the error bars using Monte Carlo simulations (Sect. 4.1); (ii) for the GASS field, we used Xspect (Tristram et al. 2005), a method that was first developed for the Archeops experiment to obtain estimates of the angular power spectrum of the CMB temperature anisotropies, including analytical error bars (Sect. 4.2). Having two completely different pipelines and a many fields with various dust and CMB contaminations (and noise contributions), is extremely valuable, as it allows us to test the robustness of our approach. 

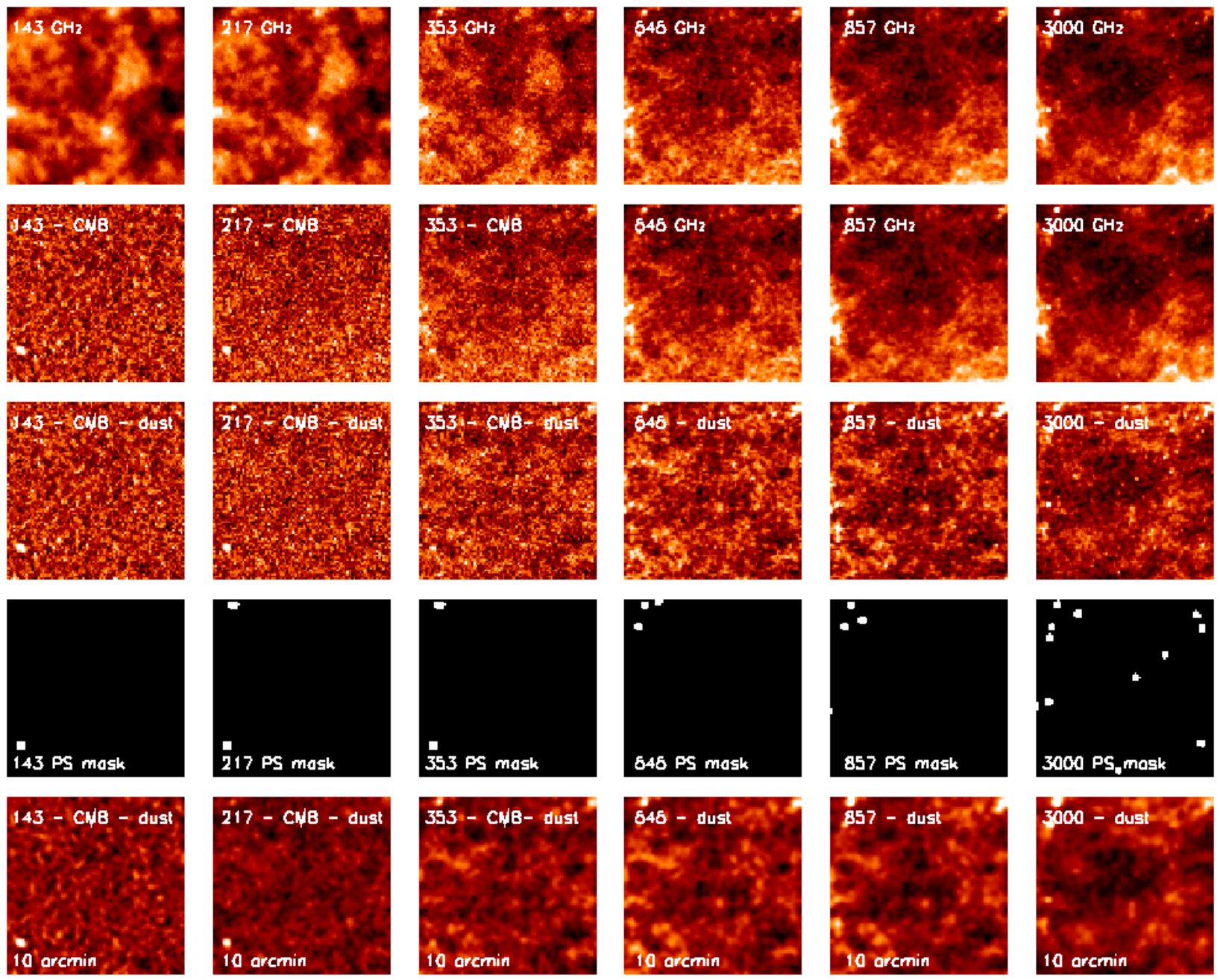

Fig. 4. Maps of the roughly $25 \mathrm{deg}^{2}$ of the SPC5 field, from left to right: 143, 217, 353, 545, 857, and $3000 \mathrm{GHz}$. From top to bottom: raw HFI and IRIS maps; CMB-cleaned maps; residual maps (CMB- and cirrus-cleaned); point source masks; and residual maps smoothed at 10' to highlight the CIB anisotropies. The joint structures clearly visible (bottom row) correspond to the anisotropies of the CIB.

\section{Angular power spectrum and bispectrum}

\subsection{Cross-correlation pipeline for the EBHIS and GBT fields}

To determine the cross-correlation between the CIB observed at two frequencies, $v_{1}$ and $v_{2}$, we used a modified version of POKER (Ponthieu et al. 2011). POKER is an algorithm that determines the power spectrum of a map, corrects for mask aliasing and computes the covariance between each power spectrum bin via a Monte Carlo approach. It has been used to measure the power spectrum of the CIB as observed by Planck-HFI (Planck Collaboration XVIII 2011) and is described in detail therein. In the following, we will call auto-power spectrum $C_{\ell}^{v_{1}}$ the usual angular power spectrum and cross-power spectrum $C_{\ell}^{v_{1} v_{2}}$ its generalization to two different frequencies defined as

$\frac{1}{2}\left\langle a_{\ell m}^{v_{1}} a_{\ell^{\prime} m^{\prime}}^{\nu_{*} *}+a_{\ell m}^{v_{1} *} a_{\ell^{\prime} m^{\prime}}^{\nu_{2}}\right\rangle=(2 \pi)^{2} C_{\ell}^{v_{1} \nu_{2}} \delta_{\ell \ell^{\prime}} \delta_{m m^{\prime}}$,

where $a_{\ell m}^{v}$ are the Fourier coefficients of the CIB anisotropy at the observation frequency $v$. We take a common mask for both frequencies and it is straightforward to generalize the algebra presented in Ponthieu et al. (2011) and Planck Collaboration XVIII (2011) to obtain an unbiased estimate of the cross-power spectrum:

$C_{b}^{v_{1} \nu_{2}} \simeq \sum_{b^{\prime}} M_{b b^{\prime}}^{-1} \hat{P}_{b^{\prime}}^{v_{1} \nu_{2}}-N_{b}^{v_{1} \nu_{2}, \text { instr }}-N_{b}^{v_{1} \nu_{2}, \text { res }}$
Here $\hat{P}_{b}^{v_{1} v_{2}}$ denotes the binned pseudo-cross-power spectrum of the maps at frequencies $v_{1}$ and $v_{2}$, and $M_{b b^{\prime}}$ is the so-called mode-mixing matrix that is described in detail in Eq. (24) of Planck Collaboration XVIII (2011) and its appendix. We recall that it includes the mode-coupling effects induced by the mask, the smoothing by the instrumental beam, and the map pixelization (see below). The two noise terms, $N_{b}^{\nu_{1} \nu_{2} \text {, instr }}$ and $N_{b}^{v_{1} \nu_{2} \text {, res }}$, refer, respectively, to the instrument noise and to the contribution of what can be considered as random components at the map level, such as the CMB residuals of our component separation and the noise of the $100 \mathrm{GHz}$ map that propagates to our maps (see Sect. 3.1). Of course, Eq. (16) applied to $v_{1}=v_{2}$ gives the auto-power spectrum of the anisotropy, and we computed the two auto-spectra at $v_{1}$ and $v_{2}$ at the same time as the cross-power spectrum.

We built very similar simulation and analysis pipelines to those of Planck Collaboration XVIII (2011) to obtain our measures and their associated error bars. We here briefly recall the main steps of these pipelines and highlight the modifications introduced by the generalization from auto-power spectra estimation to that of cross-power spectra.

\section{Analysis pipeline.}

1. In order to combine our measurements from different fields, we define a common multipole binning. Above $\ell \sim 200$, we 


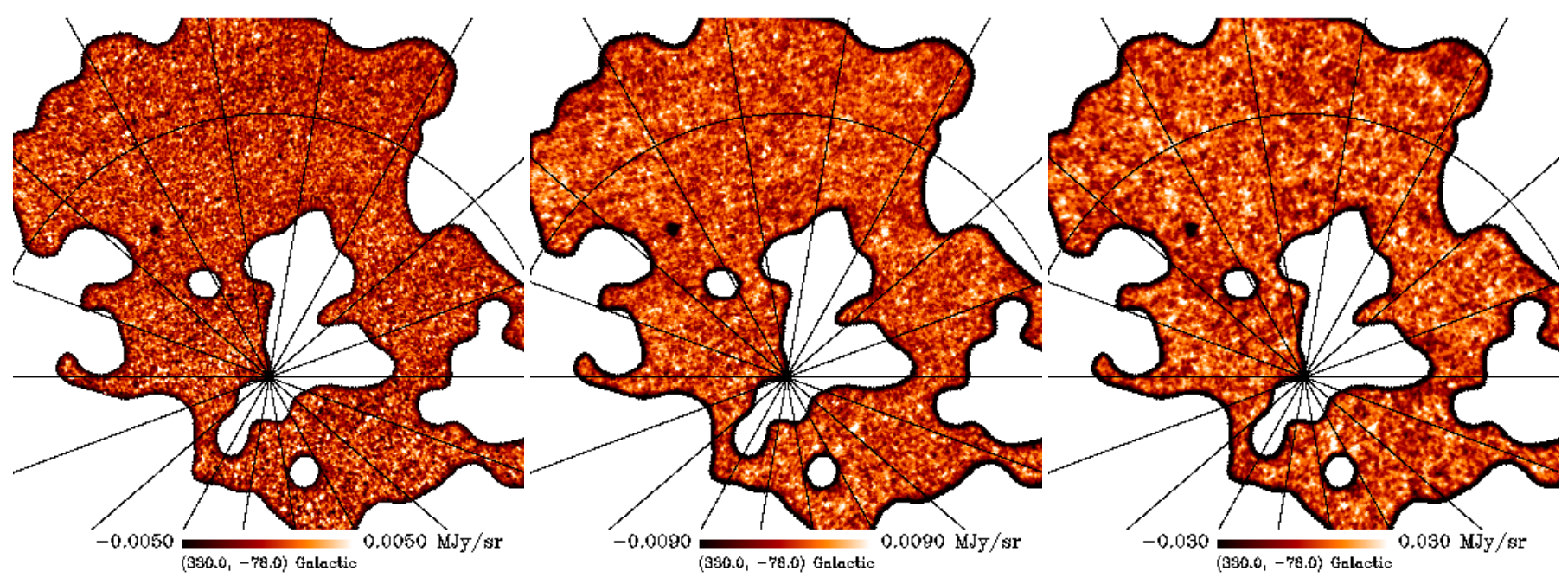

$545 \mathrm{GHz}$

$857 \mathrm{GHz}$

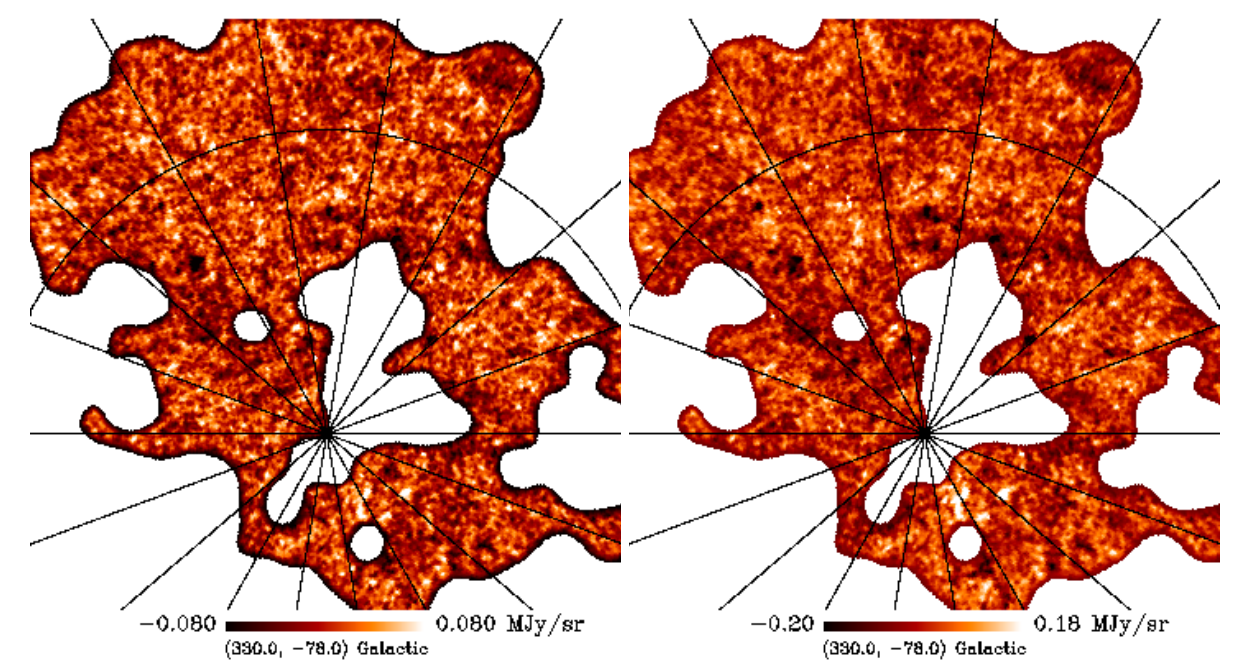

Fig. 5. Residual maps (Galactic dust and CMB removed) at $16^{\prime} .2$ angular resolution, extracted from the area covered by the GASS Hi data (on Mask1). The patch covers $60^{\circ} \times 60^{\circ}$.

choose a logarithmic binning, $\Delta \ell / \ell=0.3$, while below $\ell \sim$ 200 , we respect the generic criterion that bins of multipoles should be larger than twice the multipole corresponding to the largest angular scale contained in the field.

2. We define a common weight map $W^{v_{1} v_{2}}$ that masks out bright point sources found at both frequencies. Together with the mask, three different transfer functions must be accounted for in the computation of $M_{b b^{\prime}}$ (Eq. (24) of Planck Collaboration XVIII 2011): (i) the instrument beam transfer function that depends on the exact beam shape and the scan pattern on the observed field, although since the variation of beam transfer function is less than $1 \%$ between our fields, we take the same one for all of them (see Sect. 2.1); (ii) the HEALPix pixel window function; and (iii) the reprojection from HEALPix maps to the flat-sky maps used by POKER. The first transfer function comes from a dedicated analysis (Planck Collaboration VII 2014). The second one is provided by the HEALPix library. The third one is computed via Monte Carlo simulations: we simulate full sky HEALPix maps of diffuse emission with a typical CIB power spectrum, reproject them on our observed patches and compute the angular power spectra; the ratio between the measured and the input power spectrum gives the transfer function. This ratio is the same for all fields (all in SFL projection with 3.5' pixels) within statistical error bars, so we compute the average and apply it to all fields.

3. An estimate of the noise auto-power spectrum at $v_{1}$ and $v_{2}$ is obtained from jack-knife maps. Indeed, at each frequency, two maps can be built using only the first (respectively, the second) half of each Planck observation ring. The difference between these maps is dominated by instrumental noise. Applying POKER to these difference maps gives an estimate of the instrument noise auto-power spectrum at each frequency $N_{b}^{v_{i}}$, instr . The noise contribution to the auto-power spectrum are negligible at high frequencies.

4. $N_{b}^{v_{1} v_{2} \text {, res }}$ is the contribution of CMB residuals both from the component separation and from the propagation of noise in the $100 \mathrm{GHz}$ CMB map. We have estimates of each component of this residual power spectrum in $\ell$ space on the sphere (see. Sect. 3.1) and combine them into our measurement bins. 
Table 4. Example of a power spectrum averaged for the flat fields.

\begin{tabular}{cccccccc}
\hline \hline \multicolumn{7}{c}{$857 \mathrm{GHz} \times 857 \mathrm{GHz}$} \\
\hline$\ell_{\text {cent }}$ & $\ell_{\min }$ & $\ell_{\max }$ & $\begin{array}{c}C_{\ell} \\
{\left[\mathrm{Jy}^{2} \mathrm{sr}^{-1}\right]}\end{array}$ & $\begin{array}{c}\text { Total error } \\
{\left[\mathrm{Jy}^{2} \mathrm{sr}^{-1}\right]}\end{array}$ & $\begin{array}{c}\text { Cirrus error } \\
{\left[\mathrm{Jy}^{2} \mathrm{sr}^{-1}\right]}\end{array}$ & \multicolumn{2}{l}{ Systematic errors $\left[\mathrm{Jy}^{2} \mathrm{sr}^{-1}\right]$} \\
\cline { 5 - 8 } & & & & Projection & Beam \\
\hline 53 & 23 & 84 & $1.49 \times 10^{6}$ & $1.27 \times 10^{6}$ & $1.12 \times 10^{6}$ & $1.99 \times 10^{3}$ & $2.31 \times 10^{1}$ \\
114 & 84 & 145 & $6.37 \times 10^{5}$ & $1.62 \times 10^{5}$ & $1.16 \times 10^{5}$ & $1.84 \times 10^{3}$ & $4.48 \times 10^{1}$ \\
187 & 145 & 229 & $2.87 \times 10^{5}$ & $3.70 \times 10^{4}$ & $2.73 \times 10^{4}$ & $8.00 \times 10^{2}$ & $5.29 \times 10^{1}$ \\
320 & 229 & 411 & $1.34 \times 10^{5}$ & $8.04 \times 10^{3}$ & $5.63 \times 10^{3}$ & $4.55 \times 10^{2}$ & $6.70 \times 10^{1}$ \\
502 & 411 & 592 & $7.20 \times 10^{4}$ & $2.58 \times 10^{3}$ & $1.49 \times 10^{3}$ & $1.62 \times 10^{2}$ & $7.59 \times 10^{1}$ \\
684 & 592 & 774 & $4.38 \times 10^{4}$ & $1.81 \times 10^{3}$ & $6.14 \times 10^{2}$ & $8.93 \times 10^{1}$ & $6.82 \times 10^{1}$ \\
890 & 774 & 1006 & $3.23 \times 10^{4}$ & $9.48 \times 10^{2}$ & $2.85 \times 10^{2}$ & $9.41 \times 10^{1}$ & $6.07 \times 10^{1}$ \\
1158 & 1006 & 1308 & $2.40 \times 10^{4}$ & $4.88 \times 10^{2}$ & $1.29 \times 10^{2}$ & $5.23 \times 10^{1}$ & $4.52 \times 10^{1}$ \\
1505 & 1308 & 1701 & $1.83 \times 10^{4}$ & $2.58 \times 10^{2}$ & $5.97 \times 10^{1}$ & $2.49 \times 10^{1}$ & $3.41 \times 10^{1}$ \\
1956 & 1701 & 2211 & $1.46 \times 10^{4}$ & $1.63 \times 10^{2}$ & $2.77 \times 10^{1}$ & $2.09 \times 10^{1}$ & $4.16 \times 10^{1}$ \\
2649 & 2211 & 3085 & $1.16 \times 10^{4}$ & $1.38 \times 10^{2}$ & $1.12 \times 10^{1}$ & $7.89 \times 10^{1}$ & $6.52 \times 10^{1}$ \\
\hline
\end{tabular}

Notes. This illustrates the order of magnitude of the different errors. The total error contains the cosmic variance and instrumental noise, and also the cirrus residuals (CMB is negligible at this frequency). The contribution of cirrus errors only is given in the sixth column for comparison. The errors linked to the projection of the fields on the tangential plane and those due to the beam, although systematic and not stochastic, are given for convenience in the same units as the total power $C_{\ell}$, in Cols. 7 and 8 , respectively.

5. We now apply Eq. (16) to our data and determine their autoand cross-power spectra.

Simulation pipeline. The simulation pipeline is essential to provide the error bars on our estimates coming from the analysis pipeline. We created 100 simulations of our data maps and computed their auto- and cross-power spectra. The dispersion of these spectra gives the complete covariance matrices of our binned auto- and cross-power spectra and their associated error bars. For each realization we follow these steps.

1. The measured auto- and cross-power spectra are used as inputs to simulate CIB anisotropy maps at each frequency, with the appropriate correlated component. To do so, we generate random Gaussian amplitudes $x_{\ell}$ and $y_{\ell}$ in Fourier space, such that:

$$
\begin{aligned}
& a_{\ell}^{\nu_{1}}=x_{\ell}\left(C_{\ell}^{v_{1}}\right)^{1 / 2} ; \\
& a_{\ell}^{\nu_{2}}=x_{\ell} C_{\ell}^{v_{1} v_{2}} /\left(C_{\ell}^{v_{1}}\right)^{1 / 2}+y_{\ell}\left\{C_{\ell}^{\nu_{2} v_{2}}-\left(C_{\ell}^{\nu_{1} v_{2}}\right)^{2} / C_{\ell}^{v_{1}}\right\}^{1 / 2} .
\end{aligned}
$$

2. For each frequency $v_{i}$, we simulate a noise map with the appropriate power spectrum $\hat{N}_{\ell}^{v_{i} \text {, instr }}$ and add it to the simulated signal map.

3. We add the simulations of $\mathrm{CMB}$ and $100 \mathrm{GHz}$ noise residuals (Eqs. (1) and (2)), and of CMB residuals induced by relative calibration errors, so that we have a final pair of maps at $v_{1}$ and $v_{2}$ that are a faithful representation of our data.

Error estimation. Statistical uncertainties due to instrument noise and component separation residuals are derived using the simulation pipeline. Systematic uncertainties include mask aliasing effects, imperfect subtraction of foreground templates, and beam and projection transfer function errors. We studied some fields in common with Planck Collaboration XVIII (2011), with very similar masks, and showed that the correction of mask effects by POKER leads to no more than $2 \%$ uncertainty on the result.

The transfer function due to the projection of spherical HEALPix maps to our square patch is determined via Monte Carlo (see step 2 of the description of the analysis pipeline).
This process provides estimates $F_{b}$ of the transfer function in our measurement bins. However, we need an estimate of the transfer function $F_{\ell}$ for all $\ell$ modes, to include it in the derivation of $M_{b b^{\prime}}$. We therefore construct a smooth interpolation of our measures. The projection transfer function plays the same role as an extra instrumental beam and enters the derivation of $M_{b b^{\prime}}$. Roughly speaking, it damps the signal at high $\ell$ and correcting for this effect corresponds to dividing the measured power spectrum by the damping function. The statistical uncertainty on the determination of $F_{\ell}$ is smaller than $1 \%$, and hence leads to the same uncertainty on the $C_{\ell}$.

Between the first analysis of CIB anisotropies with PlanckHFI and this work, much progress has been achieved on the beam measurements (Planck Collaboration VII 2014). Based on this progress, we have better estimates of the beam shapes and better assessments of the uncertainties on these beam transfer functions, both for auto-spectra and for cross-spectra between different frequency bands. We used the eigenmodes to compute the beam transfer function uncertainties, which are all smaller than $0.55 \%$ in our angular range. On the contrary, auto- and cross-spectra involving IRIS suffer from a much larger beam uncertainty $\left(0.5^{\prime}\right.$ for a FWHM of $\left.4.3^{\prime}\right)$.

The systematic uncertainties on the contribution of Galactic dust residuals (see Sect. 3.2.2) are added linearly to the statistical error bars. An example of the error budget is given in Table 4.

\subsection{Cross-correlation pipeline for the GASS field}

For the large GASS field, we used another strategy:

- Due to the size of the field, which violates the flat-sky approximation used for the EBHIS and GBT fields, we compute the angular auto- and cross-power spectra on the HEALPix maps, using the Xspect algorithm (Tristram et al. 2005). We apply the Galactic dust mask discussed in Sect. 3.2.1. The power spectra are computed using maps at the HFI angular resolution $\left(N_{\text {side }}=2048\right)$.

- As the angular resolution of the HI map is $16^{\prime}$, we use a hybrid method to remove the Galactic dust. On large angular scales $(\ell \leq 590)$, we remove the cirrus from the maps using HI. On small angular scales $(\ell>590)$, we remove an estimate of the dust power spectrum. This estimate comes 


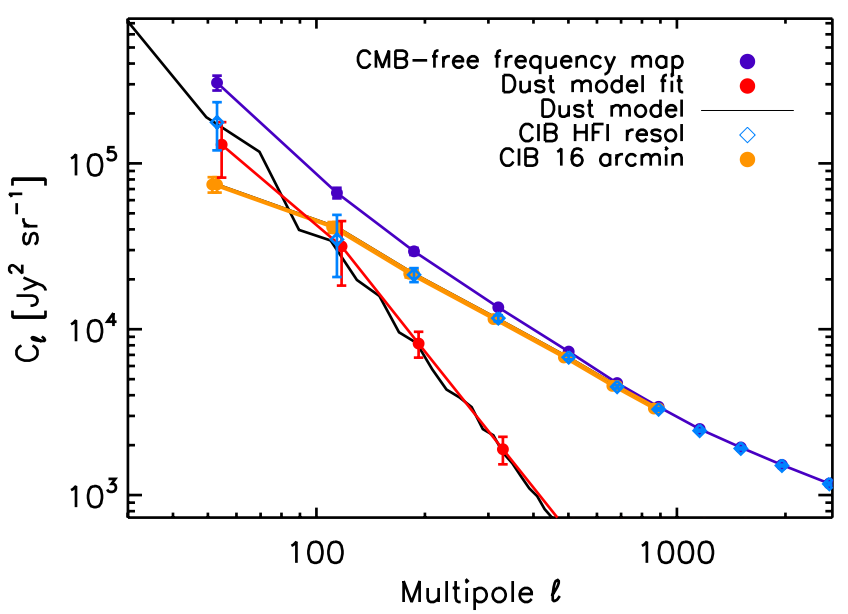

Fig. 6. $353 \times 545$ cross-power spectrum in the GASS field (using Mask1). The black line is the cross-power spectrum of the dust model, with error bars not shown for clarity. The red points are the result of a power-law fit to the dust model, using the bins of the CIB power spectrum. The CIB obtained by the spatial removal of the dust is shown in orange (note that it stops at $\ell \simeq 1000$ due to the angular resolution of the HI data), while the CIB obtained from the spectral removal of the dust (i.e., on the power spectrum) is shown in light blue (diamonds). We see that the two methods give identical CIB for $\ell \gtrsim 300$. In dark blue is the cross-power spectrum of the CMB-free frequency maps; the dust removal is negligible for $\ell \geq 700$. For clarity the points have been shifted by $\ell \pm 3 \%$ and the error linked to the cirrus bias (see Sect. 3.2.2) have not been added.

from the dust model that is fit on large angular scales $(120 \leq$ $\ell \leq 590$ ) by a power law, following Miville-Deschênes et al. (2007), and then extrapolated to small angular scales. The power-law fit has been shown to be valid for the whole range of angular scales covered by our measurements (e.g., Miville-Deschênes et al. 2010).

- As for the flat-sky fields, the CMB is removed as described in Sect. 3.1.

- Statistical error bars are not computed using simulations but using analytical formulations (see below).

Auto- and cross-power spectra. We used the maps built from the first and second halves of each pointing period. As described in Tristram et al. (2005), the spherical harmonic coefficients from the cut-sky maps are corrected from the mode-coupling introduced by the mask, as well as the beam smoothing effect in the harmonic domain. The cross-power spectra are unbiased estimates of the angular power spectrum, avoiding any correction for the instrument noise, contrary to our measurements done in Sect. 4.1. We computed the spectra using the same multipole binning as that of the flat fields. We checked that the bin to bin correlation was smaller than $1 \%$.

We show in Fig. 6 an example of a cross-power spectrum $(545 \times 353)$ computed on maps for which the CMB has been removed. We also show the power spectrum of the dust model, fitted by a broken power law. The slope of the dust power spectrum appears to flatten at $\ell \sim 110$, with a slope in the range -2.1 to -1.8 for $\ell<110$, and -2.8 to -2.7 for $\ell>110$, depending on the frequency. We compare the CIB power spectrum obtained by removing the dust at the map level to that obtained by subtracting the fit of the dust model power spectrum. For $\ell \geq 200$ we have excellent agreement. We also see that for $\ell \geq 700$ the dust removal has a negligible impact. At low $\ell$, where the dust correction is important, we chose to use the dust removal on the map, since it leads to a lower variance on the residual power spectrum, as shown in Pénin et al. (2012b). Indeed, the spatial subtraction removes each moment of the statistics, whereas the subtraction of the power spectrum only removes the first two moments. At low $\ell$, errors on the fit are also quite large. We arbitrary decide to take the transition between the dust removal on the map and on the power spectrum at $\ell=510$. The exact choice of the multipole for the transition has no consequence on the resulting CIB power spectrum.

Error bars. We quadratically combine the following error terms to obtain the final uncertainty on the GASS CIB power spectra:

- The error bars on the spectrum computed analytically as described in Tristram et al. (2005), from the auto-power and cross-power spectra of the two maps. They include both the sampling variance (which dominates at large scales) and the instrumental noise (which dominates at small scales).

- The errors linked to the CMB removal. Errors on the extra instrumental noise that have been introduced by the CMB removal (see Eq. (1)) are computed using the errors on the noise measurements at $100 \mathrm{GHz}$. For the error on the CMB contribution that is left close to the angular resolution of the 143 and, 217 and $353 \mathrm{GHz}$ channels (see Eq. (2)), we use the theoretical cosmic variance estimate on the determination of the CMB power spectrum. Finally, we add the errors that come from the relative photometric calibration in the CMB removal.

As detailed in Sect. 3.2.2, the uncertainty on the dust model is derived from simulations. It is added linearly to the statistical error bars. For $\ell \geq 510$, we also add the error on the dust model fit. Beam errors are computed using the eigenmodes. They are the same as those detailed in Sects. 2.1 and 4.1.

\subsection{Bispectrum pipeline for the GASS field}

The bispectrum $b_{\ell_{1} \ell_{2} \ell_{3}}$ is the 3-point correlation function in harmonic space:

$\left\langle a_{\ell_{1} m_{1}} a_{\ell_{2} m_{2}} a_{\ell_{3} m_{3}}\right\rangle=b_{\ell_{1} \ell_{2} \ell_{3}} \times G_{\ell_{1} \ell_{2} \ell_{3}}^{m_{1} m_{2} m_{3}}$,

with $G_{\ell_{1} \ell_{2} \ell_{3}}^{m_{1} m_{2} m_{3}}$ the Gaunt integral (Spergel \& Goldberg 1999). It is a lowest-order indicator of the non-Gaussianity of the field.

The maps used are the same as for the power spectrum analysis, but degraded to $N_{\text {side }}=512$. As the $\mathrm{S} / \mathrm{N}$ for bispectra is quite low compared to that of power spectra, bispectra have to be measured on the largest possible clean area of the sky. Here, we measure the bispectrum on GASS using Mask2. We apply the binned bispectrum estimator described in Lacasa et al. (2012) and used for the Planck tSZ map analysis (Planck Collaboration XXI 2014) and non-Gaussianity constraints (Planck Collaboration XXIV 2014). A large bin size $\Delta \ell=128$ has been adopted to minimize multipole correlations due to the mask. We used a multipole range $\ell_{\min }=129$ to $\ell_{\max }=896$, leaving six multipole bins and 43 bispectrum configurations $\left(\ell_{1}, \ell_{2}, \ell_{3}\right)$ (accounting for permutation invariance and the triangular condition). We only considered auto-bispectra for simplicity, i.e., at a single frequency (see Table D.3).

For each frequency, we computed the auto-bispectra of the two maps built using the two half-pointing period rings, and average these bispectra for a raw estimate. The raw estimate has been then debiased from mask and beam effects using simulations. Specifically we generated simulations with a high level 
of non-Gaussianity and a bispectrum corresponding to the CIB prescription from Lacasa et al. (2012), computed the ratio of the bispectrum of the masked map to the full-sky bispectrum, and finally averaged this ratio over simulations, finding a quick convergence especially at high multipoles. We found this ratio to be very close to $f_{\text {sky }} b_{\ell_{1}}(v) b_{\ell_{2}}(v) b_{\ell_{3}}(v)$ (with $b_{\ell}(v)$ the beam window function), showing that the multipole correlation is indeed negligible for this bin size and bispectrum. We checked on the two half maps that the Planck noise is close to Gaussian, hence it does not bias our bispectrum estimates; it however increases their variance.

The error estimates are the sum in quadrature of:

- cosmic variance computed with analytical formulae and including the noise;

- dust residuals from (the absolute value of) the bispectrum of the dust model, scaled to the residual dust amplitude found in Sect. 3.2.2.

Note that beam errors are completely negligible in the range of $\ell$ considered. The full-sky cosmic variance of the bispectrum is composed of four terms:

$\operatorname{Cov}\left(b_{\ell_{1} \ell_{2} \ell_{3}}, b_{\ell_{1}^{\prime} \ell_{2}^{\prime} \ell_{3}^{\prime}}\right)=\mathrm{C}_{2 \times 2 \times 2}+\mathrm{C}_{3 \times 3}+\mathrm{C}_{2 \times 4}+\mathrm{C}_{6}$,

with (see Lacasa et al. 2012)

$$
\mathrm{C}_{2 \times 2 \times 2}=\frac{C_{\ell_{1}} C_{\ell_{2}} C_{\ell_{3}}}{\frac{(2 \ell+1)_{123}}{4 \pi}\left(\begin{array}{ccc}
\ell_{1} & \ell_{2} & \ell_{3} \\
0 & 0 & 0
\end{array}\right)^{2}} \delta_{\ell_{1} \ell_{1}^{\prime}} \delta_{\ell_{2} \ell_{2}^{\prime}} \delta_{\ell_{3} \ell_{3}^{\prime}} \times \Delta_{\ell_{1} \ell_{2} \ell_{3}} .
$$

Here

$$
\Delta_{\ell_{1} \ell_{2} \ell_{3}}= \begin{cases}6 & \text { equilateral triangles } \\ 2 & \text { isosceles triangles } \\ 1 & \text { general triangles }\end{cases}
$$

and when the bispectrum is factorizable

$$
\begin{aligned}
\mathrm{C}_{3 \times 3}=b_{\ell_{1} \ell_{2} \ell_{3}} b_{\ell_{1}^{\prime} \ell_{2}^{\prime} \ell_{3}^{\prime}} \times & \left(\frac{\delta_{\ell_{1} \ell_{1}^{\prime}}}{2 \ell_{1}+1}+\frac{\delta_{\ell_{1} \ell_{2}^{\prime}}}{2 \ell_{1}+1}+\frac{\delta_{\ell_{1} \ell_{3}^{\prime}}}{2 \ell_{1}+1}\right. \\
& +\frac{\delta_{\ell_{2} \ell_{1}^{\prime}}}{2 \ell_{2}+1}+\frac{\delta_{\ell_{2} \ell_{2}^{\prime}}}{2 \ell_{2}+1}+\frac{\delta_{\ell_{2} \ell_{3}^{\prime}}}{2 \ell_{2}+1} \\
& \left.+\frac{\delta_{\ell_{3} \ell_{1}^{\prime}}}{2 \ell_{3}+1}+\frac{\delta_{\ell_{3} \ell_{2}^{\prime}}}{2 \ell_{3}+1}+\frac{\delta_{\ell_{3} \ell_{3}^{\prime}}}{2 \ell_{3}+1}\right)
\end{aligned}
$$

$\mathrm{C}_{2 \times 4}$ and $\mathrm{C}_{6}$ involve, respectively, the map trispectrum and 6 -point function. $\mathrm{C}_{2 \times 2 \times 2}$ is the cosmic variance in the weak nonGaussianity limit, as considered, e.g., by Crawford et al. (2014) in their tSZ and CIB non-Gaussianity study; it produces a diagonal covariance matrix, since it does not couple the different configurations. $\mathrm{C}_{3 \times 3}$ is the bispectrum correction to the covariance matrix and couples bispectrum estimates in different configurations. In the cosmic variance limited case, we found that including only $\mathrm{C}_{2 \times 2 \times 2}$ would noticeably overestimate the detection significance. However when considering all error sources, $\mathrm{C}_{3 \times 3}$ has a small impact on the $\mathrm{S} / \mathrm{N}$; hence we include it in the following analysis but neglect higher order corrections $C_{2 \times 4}$ and $C_{6}$.

We use the $f_{\text {sky }}$ approximation, that is $\operatorname{Cov}=\operatorname{Cov}_{\text {full-sky }} / f_{\text {sky }}$, as the first multipoles were discarded and we saw no mode coupling with our large bin size. The power spectrum used for $\mathrm{C}_{2 \times 2 \times 2}$ is the map auto-power spectrum, including the noise as necessary, debiased from the mask and beam effects.

As described in Sect. 3.1.2, the residual maps are contaminated by $\mathrm{tSZ}$ contribution at $100 \mathrm{GHz}$ leaking through the $\mathrm{CMB}$ cleaning process. While this contamination is negligible compared to the CIB signal at $353 \mathrm{GHz}$ and above, it is important at $217 \mathrm{GHz}$, where the contamination can be as large as $40 \%$, depending on the configuration and multipole. We derived the SZ bispectrum by measuring the bispectrum of the map of the Planck SZ cluster catalogue (both clusters and candidate clusters from Planck Collaboration XXX (2014) are considered). An $80 \%$ error on the amplitude of the tSZ correction is added to the covariance measurement described previously. This error is based on the relative difference between the bispectrum of the Planck SZ catalogue of confirmed clusters (Planck Collaboration XXX 2014), the bispectrum of the Planck estimated tSZ map, and the bispectrum of the Planck FFP6 SZ simulations (see the bispectrum analysis in Planck Collaboration XXI 2014). This error estimate is conservative, since it takes the maximum observed difference for all multipoles and configurations.

\subsection{CIB power spectrum}

Figure 7 presents a summary of all the measured auto- and crosspower spectra on residual maps. Following the same covariance studies as in Planck Collaboration XVIII (2011), we combine our cross-power spectrum estimates on individual fields $f$ for each bin $b$ into an average cross-power spectrum, using inverse variance weights,

$C_{b}^{v \times v^{\prime}}=\frac{\sum_{f} W_{b}^{f} P_{b}^{f, v \times v^{\prime}}}{\sum_{f} W_{b}^{f}}$

with $W_{b}^{f}=1 / \sigma^{2}\left(P_{b}^{f, v \times v^{\prime}}\right)$. These weights are estimated in the following way:

- Step 1: only statistical errors are used to compute a weighted average of the power spectra and their associated error bars on small fields.

- Step 2: the projection error is added linearly to the error bar of step 1 .

- Step 3: for frequencies where observations on GASS are available, we average the GASS power spectrum and the small fields power spectrum. We here use inverse statistical variance weights for GASS, and the inverse variance derived from step 2 for the small fields. This gives the final power spectrum estimate and a pseudo-statistical error bar.

- Step 4: we compute the error due to beam uncertainties using the average power spectrum. We add this error linearly to the error derived on Step 3.

- Step 5: the bias induced by Galactic dust residuals is linearly added to the error derived on Step 4 to obtain the final total error bar.

The resulting power spectra and their errors are given in Table D.1. For the $217 \times 217$ auto-power spectrum, only the measurement on the GASS field is considered, as the measurement in the flat-sky fields is noise dominated. Note also that the last bin for all measurements involving the subtraction of the $100 \mathrm{GHz} \mathrm{CMB}$ template is $\ell=1956$, due to the angular resolution of Planck-HFI at $100 \mathrm{GHz}$. To obtain the CIB power spectra, the estimates obtained from the CMB- and dust-free maps (Table D.1) have to be corrected for SZ contamination (Eqs. (5) and (9)), and for the spurious CIB contamination (Eq. (4)) induced by our CMB template. This last contaminant is computed using our best-fit model described in Sect. 5.5. Moreover, following Sects. 3.2.2 and 3.2.3, the first two bins at multipoles $\ell=53$ and 114 have to be considered as upper limits. CIB power 


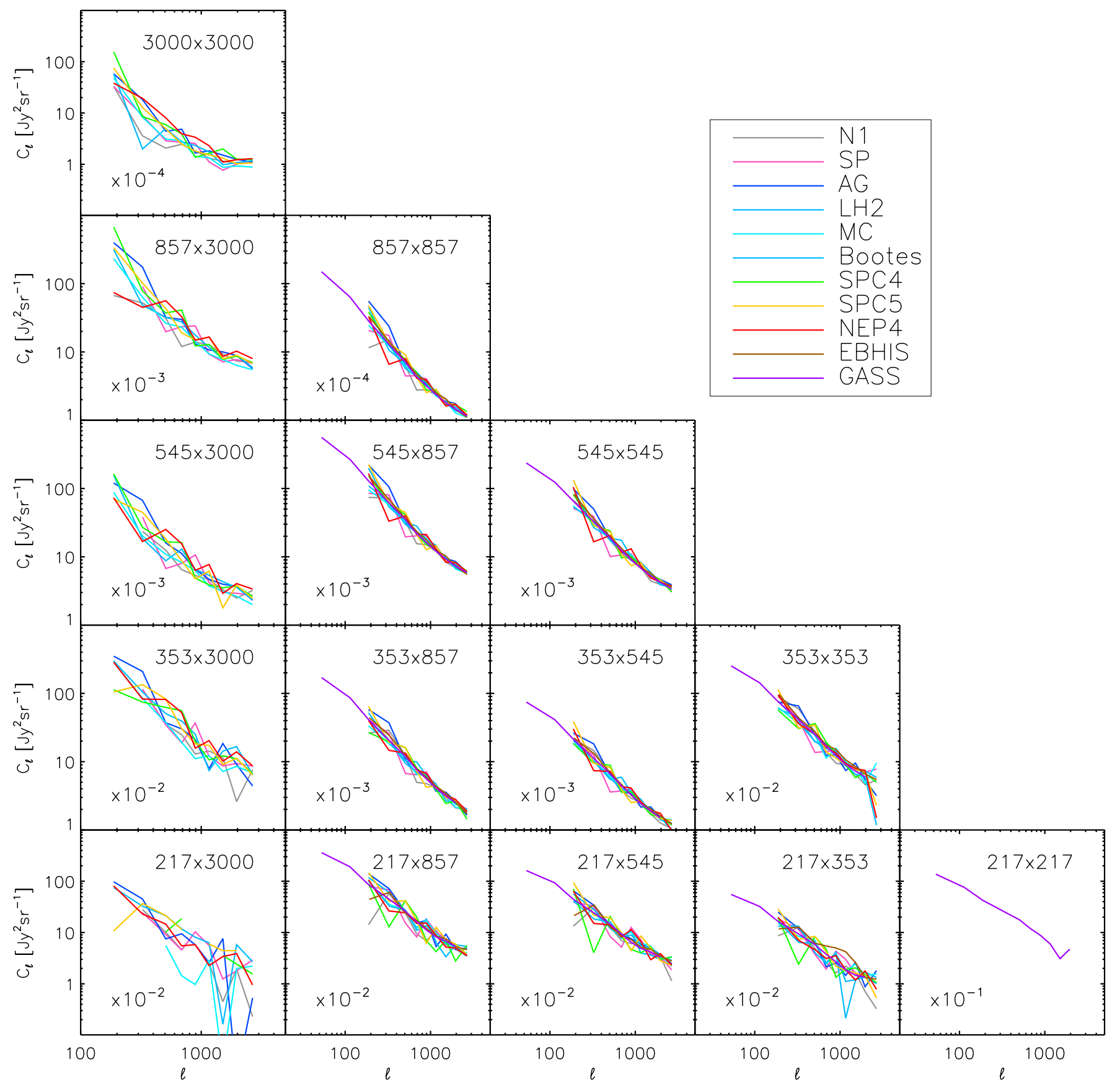

Fig. 7. Auto- and cross-power spectra of the CIB for each field (but EBHIS, Bootes and GASS at $3000 \mathrm{GHz}$, see Sect. 3.3). For readability, error bars on individual measurements are not plotted. For the $217 \times 217$ case, measurements on the flat-sky fields are noise dominated, and we thus use only the results from the GASS field. For display purpose, power spectra have been multiplied by the number given at the bottom-left side of each panel.

spectrum values are given in Table D.2. The errors contain all the terms: statistical uncertainty; beam and projection uncertainty; cirrus bias; and errors from the SZ correction. CIB power spectra, comparing the measurements with the model are presented later (Fig. 12). Comparison with previous recent measurements are shown and discussed in Sect. 6.3.

The power spectra from the flat-sky and GASS fields have been obtained using two independent pipelines. The fields have different Galactic dust and point sources contamination, as well as instrument noise levels, and between GASS and the flat-sky fields, different pixelization and projection. Comparing combined power spectra obtained on all flat-sky fields to that obtained on GASS is thus a powerful consistency check on our determination of power spectra and error bars. We show in Fig. 8 this comparison for an arbitrary set of frequencies. The power spectra are always compatible within $1 \sigma$. Of course, due to the much larger area of the GASS field, the CIB measured in GASS has much smaller cosmic variance errors.

\subsection{CIB bispectrum}

We measure the bispectrum only at 217,353 , and $545 \mathrm{GHz}$. The bispectrum at $143 \mathrm{GHz}$ is noise-dominated and is moreover highly contaminated by tSZ and extragalactic radio point sources. At $857 \mathrm{GHz}$, as we are using Mask2, Galactic dust 


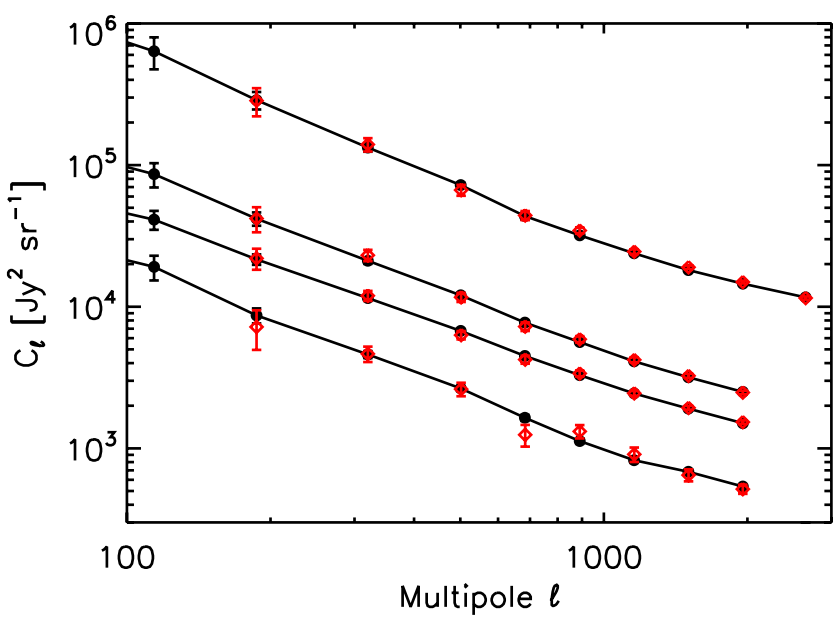

Fig. 8. Comparison between the CMB- and dust-free map power spectra obtained from the analysis of the flat fields $\left(328 \mathrm{deg}^{2}\right.$, red diamonds) and GASS (1914 $\mathrm{deg}^{2}$, black dots). From top to bottom: $857 \times 857$; $857 \times 353 ; 545 \times 353$; and $857 \times 217$.

Table 5. Detection significance of the bispectra at each frequency.

\begin{tabular}{ccc}
\hline \hline Band & Mean $S / N$ per configuration & Total $S / N$ \\
\hline $217 \mathrm{GHz}$ & 1.24 & 5.83 \\
$353 \mathrm{GHz}$ & 2.85 & 19.27 \\
$545 \mathrm{GHz}$ & 4.59 & 28.72 \\
\hline
\end{tabular}

Notes. The mean $\mathrm{S} / \mathrm{N}$ per configuration and the total $\mathrm{S} / \mathrm{N}$ are not directly linked by the square root of the configuration numbers, because the covariance matrix is not diagonal.

residuals contaminate the bispectrum in most configurations. In particular, the residuals produce a rising bispectrum at high multipoles. In the following, we are thus not considering the $857 \mathrm{GHz}$ frequency for the analysis. At 217, 353, and $545 \mathrm{GHz}$, the bispectrum is measured in 38, 40 and 36 configurations, respectively. We show in Fig. 9 the measured CIB bispectrum at $353 \mathrm{GHz}$ for some particular configurations, namely equilateral $(\ell, \ell, \ell)$, orthogonal isosceles $(\ell, \ell, \sqrt{2} \ell)$, flat isosceles $(\ell, \ell, 2 \ell)$, and squeezed $\left(\ell_{\min }, \ell, \ell\right)$. The bispectrum decreases with scale and exhibits a peak in the squeezed configurations, as predicted by Lacasa et al. (2012).

In Table 5, we give the significance of the detection for the three frequencies used, with either the average significance per configuration or the significance of the total bispectrum, when accounting for the whole covariance matrix. The bispectrum is significantly detected at each frequency individually. Moreover, these measurements represent the first detection of the CIB bispectrum per configuration, permitting us to probe the scale and configuration dependence of the bispectrum, as well as its frequency behaviour. The bispectrum values are given in Table D.3.

\section{Interpreting CIB power spectrum measurements}

Once the CMB and Galactic dust have been removed, there are three astrophysical contributors to the power spectrum at the HFI frequencies: two from dusty star-forming galaxies (with both shot noise, $C_{\mathrm{d} \text {,shot }}^{v \times v^{\prime}}$, and clustering, $C_{\mathrm{d} \text {,clust }}^{v \times v^{\prime}}(\ell)$, components); and one from radio galaxies (with only a shot-noise component, $C_{\mathrm{r} \text {,shot }}^{v \times v^{\prime}}$, the clustering of radio sources being negligible, e.g., Hall et al. 2010). The measured CIB power spectrum $C_{\text {measured }}^{v \times v^{\prime}}(\ell)$ is thus

$C_{\text {measured }}^{\nu \times v^{\prime}}(\ell)=C_{\mathrm{d}, \text { clust }}^{\nu \times v^{\prime}}(\ell)+C_{\mathrm{d}, \text { shot }}^{\nu \times v^{\prime}}+C_{\mathrm{r}, \text { shot }}^{\nu \times v^{\prime}}$.

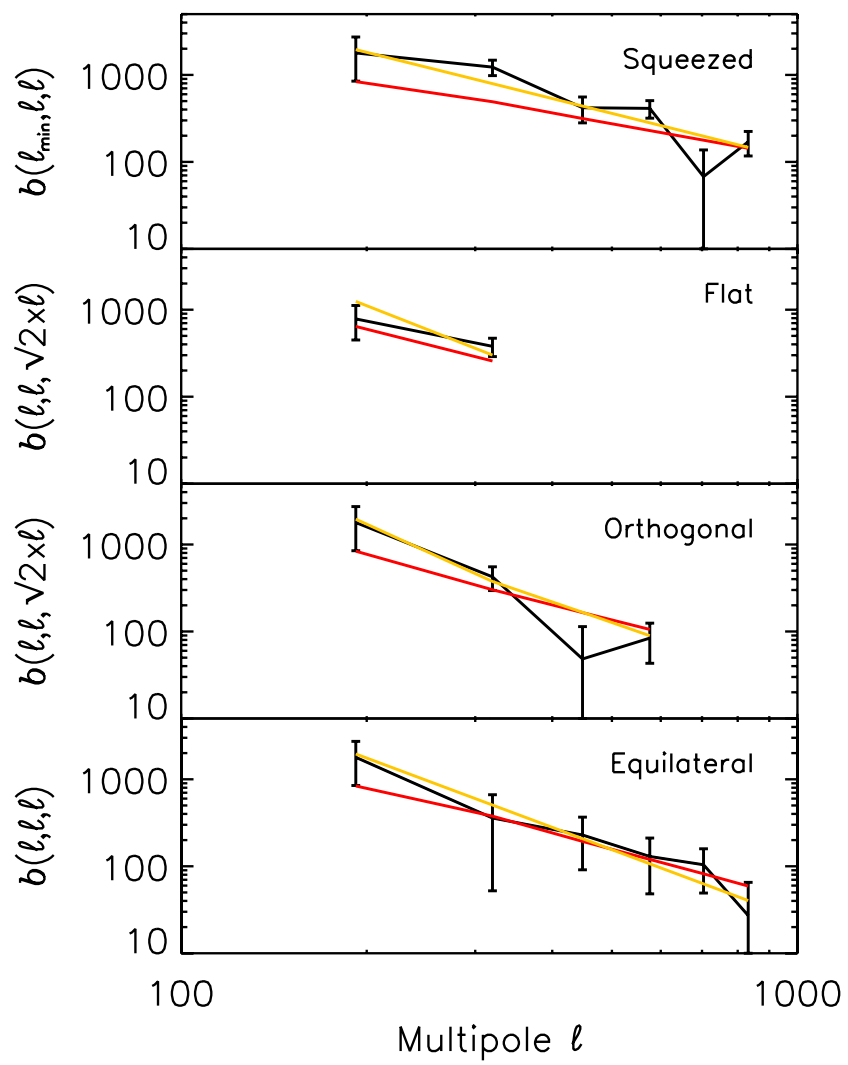

Fig. 9. CIB bispectrum at $353 \mathrm{GHz}$ in some particular configurations (black points, in $\mathrm{Jy}^{3} \mathrm{sr}^{-1}$ ). The red curve is the CIB bispectrum predicted from the power spectrum (best-fit model from Sect. 5.5) following Lacasa et al. (2012). The yellow curve is a power-law fit (as given in Table 13). See Sect. 6.5 for more details.

In this section, we first discuss the shot-noise contributions. We then describe how we can model $C_{\mathrm{d} \text {,clust }}^{v \times v^{\prime}}(\ell)$. Two approaches are considered. The first one is the simplest, and uses only the largescale CIB measurements to fit for a linear model. The second one is based on the halo-model formalism. Our main goal is to use CIB anisotropies to measure the SFRD and effective redshift evolution of the bias.

\subsection{Shot noise from dusty star forming and radio galaxies}

The shot noise arises from sampling of a background composed of a finite number of sources, and as such is decoupled from the correlated term. The angular resolution of the HFI instrument is not high enough to measure the shot-noise levels. As demonstrated in Planck Collaboration XVIII (2011), the nonlinear contribution to the power spectrum is degenerate with the shot-noise level (on the scales probed by Planck). Since our data by themselves are not sufficient to explore this degeneracy, we need to rely on a model to compute the shot noise.

\subsubsection{Auto-power spectrum}

The shot-noise level at frequency $v$ can be easily computed using monochromatic galaxy number counts. Let us consider a flux interval $\left[S_{k}, S_{k}+\Delta S_{k}\right.$ ]. The number of sources per unit solid angle, $n_{k}$ in this flux interval is

$n_{k}=\frac{\mathrm{d} N}{\mathrm{~d} S} \Delta S_{k}$ 
and the variance is,

$\sigma_{B_{k}}^{2}=n_{k} S_{k}^{2}$.

Summing all flux intervals gives the variance on the total contribution to the CIB:

$\sigma_{B}^{2}=\sum_{k} n_{k} S_{k}^{2}=\frac{\mathrm{d} N}{\mathrm{~d} S} S_{k}^{2} \Delta S_{k}$

When we take the limit of $\Delta S_{k}$ tending to zero, this sum becomes the integral

$\sigma_{B}^{2}=\int_{0}^{S^{\mathrm{c}}} \frac{\mathrm{d} N}{\mathrm{~d} S} S^{2} \mathrm{~d} S$

Here $S^{\mathrm{c}}$ is the flux cut above which bright sources are detected and can be removed. This cut is mandatory, since the integral does not converge in the Euclidian regime, $\frac{\mathrm{d} N}{\mathrm{~d} S} \propto S^{-2.5}$, which is the case at bright fluxes for star-forming galaxies.

\subsubsection{Cross-power spectrum}

We now consider two frequencies $v$ and $v^{\prime}$. The number of sources $n_{k l}$ in the flux density and redshift intervals [ $\left.S_{k}, S_{k}+\Delta S_{k}\right]$ and $\left[z_{l}, z_{l}+\Delta z_{l}\right]$, is

$n_{k l}=\frac{\mathrm{d} N}{\mathrm{~d} S \mathrm{~d} z} \Delta S_{k} \Delta z_{l}$

Considering a small redshift interval, the covariance between the two frequencies can be approximated as:

$\sigma_{v_{1} v_{2}, k l}=n_{k l} S_{v, k l} S_{v^{\prime}, k l}=n_{k l} S_{v, k l}^{2} R_{v v^{\prime}, k l}$

where $R_{v v^{\prime}, k l}$ is the mean colour for the considered galaxy population in the considered flux density and redshift interval. Using a mean colour per flux density and redshift interval is not a strong assumption as long as $\Delta S_{k}$ and $\Delta z_{l}$ are small. Summing over flux densities, redshifts and the galaxy population gives

$\sigma_{v v^{\prime}}=\sum_{\text {pop }} \sum_{k} \sum_{l} n_{\mathrm{pop}, k l} S_{v, k l}^{2} R_{v v^{\prime}, \mathrm{pop}, k l}$

with the integral limit

$$
\begin{gathered}
\sigma_{v v^{\prime}}=\sum_{\text {pop }} \int_{S_{v}=0}^{\infty} \int_{z=0}^{\infty} H\left(S_{v}<S_{v}^{\mathrm{c}}, R_{v^{\prime}, \text { pop }} S_{v}<S_{v^{\prime}}^{\mathrm{c}}\right) \\
\frac{\mathrm{d} N_{\text {pop }}}{\mathrm{d} S_{\nu} \mathrm{d} z} S_{\nu}^{2} R_{v^{\prime}, \text { pop }} \mathrm{d} S_{\nu} \mathrm{d} z .
\end{gathered}
$$

Here $H\left(P_{1}, P_{2}\right)$ is equal to 1 when $P_{1}$ and $P_{2}$ are both true, and 0 otherwise, and $S_{v}^{\mathrm{c}}$ and $S_{v^{\prime}}^{\mathrm{c}}$ are the flux cuts in the frequency bands $v$ and $v^{\prime}$. They are given in Table 1 .

We use this formalism to compute the radio galaxy shot noise. For the star-forming dusty galaxy shot noise, we rely on the formalism detailed in Béthermin et al. 2013 (their Appendix B).

\subsubsection{Shot-noise values}

We use the Béthermin et al. (2012a) model to compute the starforming dusty galaxy shot noise, $C_{\mathrm{d} \text {,shot }}^{v \times v^{\prime}}$ (Eq. (23)). The model is in rather good agreement with the number counts measured by Spitzer and Herschel (e.g., Glenn et al. 2010). It also gives a reasonable CIB redshift-distribution, which is important for the cross-spectra. Since this model is based on observations that have typical calibration uncertainties of $<8 \%$, the estimations of the shot-noise levels (being proportional to the square of calibration factor) cannot be accurate to more than about $16 \%$. Uncertainties in the flux cuts induce small variations in the shot noise (less than $3 \%$ at all frequencies). We take $20 \%$ as the shotnoise $1 \sigma$ uncertainty.

For extragalactic radio sources, we use the Tucci et al. (2011) model (more specifically, the one referred as "C2Ex" in the paper) to compute $C_{\mathrm{r} \text {,shot }}^{\nu \times v^{\prime}}$ (Eq. (23)). The predictions for highfrequency number counts are based on a statistical extrapolation of flux densities of radio sources from low-frequency data (1-5 GHz). In particular, this model considers physically based recipes to describe the complex spectral behaviour of blazars, which dominate the mm-wave counts at bright flux densities. It is able to give a good fit to all bright extragalactic radio source data available so far: number counts up to $600 \mathrm{GHz}$; and spectral index distributions up to at least 200-300 GHz (see Tucci et al. 2011; Planck Collaboration Int. VII 2013; López-Caniego et al. 2013). As for the dusty galaxies, we consider an error of $20 \%$ on the shot-noise computation from the model. But unlike for dusty galaxies, the shot noise for the radio population depends strongly on the flux cut (Planck Collaboration XVIII 2011). Accordingly, we add to the $20 \%$ mentioned above, a shot-noise error deriving from the shot-noise variations as we change the flux cut, considering the flux cut errors given in Table 1.

The shot-noise levels for the HFI flux cuts given in Sect. 2.4, are listed in Tables 6 and 7.

\subsection{Basics of CIB correlated anisotropy modelling}

The angular power spectrum of CIB correlated anisotropies is defined as:

$\left\langle\delta I_{\ell m}^{v} \delta I_{\ell^{\prime} m^{\prime}}^{v^{\prime}}\right\rangle=C_{\mathrm{d}, \mathrm{clust}}^{v \times v^{\prime}}(\ell) \times \delta_{\ell \ell^{\prime}} \delta_{m m^{\prime}}$,

where $v$ and $v^{\prime}$ denote the observing frequencies and $I^{v, v^{\prime}}$ the measured intensity at those frequencies. In a flat universe, the intensity is related to the comoving emissivity $j$ via

$$
\begin{aligned}
I^{v} & =\int \mathrm{d} z \frac{\mathrm{d} \chi}{\mathrm{d} z} \operatorname{aj}(v, z) \\
& =\int \mathrm{d} z \frac{\mathrm{d} \chi}{\mathrm{d} z} a \bar{j}(v, z)\left(1+\frac{\delta j(v, z)}{\bar{j}(v, z)}\right),
\end{aligned}
$$

where $\chi(z)$ is the comoving distance to redshift $z$, and $a=1 /(1+z)$ is the scale factor. Combining Eqs. (32) and (33) and using the Limber approximation, we obtain

$C_{\mathrm{d}, \text { clust }}^{v \times v^{\prime}}(\ell)=\int \frac{\mathrm{d} z}{\chi^{2}} \frac{\mathrm{d} \chi}{\mathrm{d} z} a^{2} \bar{j}(v, z) \bar{j}\left(v^{\prime}, z\right) P_{j}^{v \times v^{\prime}}(k=\ell / \chi, z)$,

where $P_{j}^{v \times v^{\prime}}$ is the 3D power spectrum of the emissivities and is defined as follows:

$\left\langle\delta j(\boldsymbol{k}, v) \delta j\left(\boldsymbol{k}^{\prime}, v^{\prime}\right)\right\rangle=(2 \pi)^{3} \bar{j}(v) \bar{j}\left(v^{\prime}\right) P_{j}^{v \times v^{\prime}}(\boldsymbol{k}) \delta^{3}\left(\boldsymbol{k}-\boldsymbol{k}^{\prime}\right)$.

In the context of CIB anisotropy modelling, the simplest version of the so-called "halo model", which provides one view of the large-scale structure of the Universe as clumps of dark matter, consists of equating $P_{j}$ with the galaxy power spectrum $P_{\mathrm{gg}}$. This is equivalent to assuming that the CIB is sourced equally by all galaxies, so that the spatial variations in the emissivities trace the galaxy number density,

$\delta j / \bar{j}=\delta n_{\text {gal }} / \bar{n}_{\text {gal }}$. 
A\&A 571, A30 (2014)

Table 6. Shot-noise levels $C_{\mathrm{d} \text {,shot }}^{v \times v^{\prime}}\left(\right.$ flat power-spectra) for star-forming galaxies (in $\mathrm{Jy}^{2} \mathrm{sr}^{-1}$ ) computed using the Béthermin et al. (2012a) model.

\begin{tabular}{|c|c|c|c|c|c|c|c|c|}
\hline & 3000 & 857 & 545 & & 353 & 217 & 143 & 100 \\
\hline 3000 & $10064 \pm 2013$ & & & & . & $\cdots$ & $\cdots$ & $\cdots$ \\
\hline 857 & $4427 \pm 885$ & $5628 \pm 1126$ & & & $\ldots$ & $\ldots$ & $\ldots$ & $\ldots$ \\
\hline 545 & $1525 \pm 305$ & $2655 \pm 531$ & $1454 \pm 291$ & & $\ldots$ & & $\ldots$ & $\ldots$ \\
\hline 353 & $434 \pm 87$ & $913 \pm 183$ & $543 \pm 109$ & 225 & \pm 45 & $\ldots$ & $\ldots$ & $\ldots$ \\
\hline 217 & $96 \pm$ & $216 \pm 43$ & $135 \pm 27$ & 59 & \pm 12 & $16 \pm 3$ & $\ldots$ & $\ldots$ \\
\hline 143 & $26 \pm$ & $56 \pm$ & $35 \pm 7$ & 15 & \pm 3 & $4.3 \pm 0.9$ & $1.2 \pm 0.2$ & \\
\hline 100 & $11 \pm$ & $20 \pm$ & $12 \pm 2$ & 5.4 & \pm 1.1 & $1.5 \pm 0.3$ & $0.42 \pm 0.08$ & $0.15 \pm 0.03$ \\
\hline
\end{tabular}

Notes. To obtain the shot noise in $\mathrm{Jy}^{2} \mathrm{sr}^{-1}$ for our photometric convention $v I_{v}=$ const., a colour correction, given in Sect. 5.2, has to be applied.

Table 7. Shot-noise levels $C_{\mathrm{r}, \text { shot }}^{v \times v^{\prime}}$ (flat power-spectra) for radio galaxies (in $\mathrm{Jy}^{2} \mathrm{sr}^{-1}$ ) computed using the Tucci et al. (2011) model.

\begin{tabular}{rccccccc}
\hline \hline & 3000 & 857 & 545 & 353 & 217 & 143 & 100 \\
\hline 3000 & $\ldots$ & $\ldots$ & $\ldots$ & $\ldots$ & $\ldots$ & $\ldots$ & \\
857 & $\ldots$ & $4.28 \pm 0.90$ & $\ldots$ & $\ldots$ & $\ldots$ & $\ldots$ & $\ldots$ \\
545 & $\ldots$ & $2.28 \pm 0.56$ & $2.86 \pm 0.70$ & $\ldots$ & $\ldots$ & $\ldots$ & $\ldots$ \\
353 & $\ldots$ & $2.10 \pm 0.53$ & $2.59 \pm 0.63$ & $3.28 \pm 0.82$ & $\ldots$ & $\ldots$ & $\ldots$ \\
217 & $\ldots$ & $1.53 \pm 0.46$ & $1.92 \pm 0.56$ & $2.40 \pm 0.70$ & $3.12 \pm 0.79$ & $\ldots$ & $\ldots$ \\
143 & $\ldots$ & $2.38 \pm 0.58$ & $2.86 \pm 0.68$ & $3.57 \pm 0.82$ & $3.68 \pm 0.99$ & $6.05 \pm 1.47$ & $\ldots$ \\
100 & $\ldots$ & $2.73 \pm 0.64$ & $3.24 \pm 0.73$ & $4.02 \pm 0.90$ & $4.31 \pm 1.07$ & $6.47 \pm 1.39$ & $8.47 \pm 1.97$ \\
\hline
\end{tabular}

Notes. To obtain the shot noise in $\mathrm{Jy}^{2} \mathrm{sr}^{-1}$ for our photometric convention $v I_{v}=$ const., a colour correction has to be applied. It is however lower than $1 \%$ (see Sect. 5.2).

The dark matter haloes are populated through a halo occupation distribution (HOD) prescription. Ultimately, $P_{\mathrm{gg}}(k, z)$ is written as the sum of the contributions of galaxies within a single dark matter halo (" $1 \mathrm{~h}$ ") and galaxies belonging to two different haloes ("2h"):

$P_{\mathrm{gg}}(k, z)=P_{1 \mathrm{~h}}(k, z)+P_{2 \mathrm{~h}}(k, z)$.

On large scales $P_{2 \mathrm{~h}}$ reduces to a constant bias (squared) times the linear theory power spectrum, while the 1-halo contribution encapsulates the non-linear distribution of matter.

In this paper, we developed two approaches for the modelling of CIB anisotropies.

- The first one (Sect. 5.4) is very simple, and takes advantage of the accurate measurement of CIB anisotropies with Planck and IRIS at large angular scales. As an alternative to the HOD model for $P_{\text {gg }}$ we use a constant bias model in which $P_{\mathrm{gg}}(k, z)=b_{\text {eff }}^{2} P_{\text {lin }}(k, z)$, where $b_{\text {eff }}$ is a redshift- and scale-independent bias and $P_{\text {lin }}(k)$ is the linear theory, darkmatter power spectrum.

- The second one (Sect. 5.5) uses the anisotropies at all angular scales, and takes advantage of the frequency coverage of our measurements, to constrain a halo model with a luminositymass dependence. As a matter of fact, the model described above, which assumes that emissivity density traces galaxy number density (Eq. (36)), implies that all galaxies contribute equally to the emissivity density, irrespective of the masses of their host haloes. It assumes that all galaxies have the same luminosity, which is a crude assumption, as the luminosity and the clustering strength are closely related to the mass of the host halo.

Before becoming fully immersed in the details of our model building and fitting, we want to stress that the purpose of the following sections is to build as "physical" a model as possible that reproduces our measurements. To do so, we will expand upon the large amount of work in the literature that has exploited the simplifying concept of the halo model. We hope that in this way our work will have a wider impact. Nevertheless, it has to be emphasized that these models are very phenomenological and multi-faceted. To some extent, there is no such thing as the halo model, since there are many hidden assumptions underlying the application of the idea. For example, in our approach we will rely on a concentration prescription as a function of mass and redshift, and use this all the way to a redshift of 6 , thus pushing into a regime where the model has not previously been validated. The same holds for our ansatz for the $L-M$ relation and more generally to the concept of the HOD. To explore the dependence of our conclusions on these hidden assumptions is a task that goes well beyond the scope of the current paper and would certainly require a much more extensive use of simulations. It would of course be possible to weaken some of the assumptions by including them as Bayesian priors in our fit - but that would simply be side-stepping some of the serious conceptual limitations which exist here. For these reasons, we chose, in this current modelling effort, to simply make our assumptions clear and justify our choice of fixing values when possible. This approach is well-defined, but leaves a degree of uncertainty unaccounted for in the error budget, which should be kept in mind when interpreting the results, particularly in terms of physical parameters. Uncertainties in parameters would certainly be expected to increase if we relax some of the model's assumptions.

\subsection{Fitting for a model}

The power spectra that are computed by the models need to be colour-corrected, from a CIB SED to our photometric convention $v I_{v}=$ const. We use the CIB SED from Béthermin et al. (2012a) to compute the colour corrections. They are equal to $1.076,1.017,1.119,1.097,1.068,0.995$, and 0.960 at 100, 143, $217,353,545,857$, and $3000 \mathrm{GHz}$, respectively. The correction to the power spectra follows

$C_{\ell, v, v^{\prime}}^{\text {model }} \times \mathrm{cc}_{v} \times \mathrm{cc}_{v^{\prime}}=C_{\ell, v, v^{\prime}}^{\text {measured }}$. 
We use the same colour corrections (cc) for both the star-forming galaxy shot noise and the CIB power spectrum. For the radio galaxy shot noise we use a power law $S_{v} \propto v^{\alpha}$, with $\alpha=-0.5$. This is the average spectral index for radio sources that mainly contribute to the shot-noise power spectra. With such an SED, we find that the colour corrections are all lower than $0.7 \%$ for $100 \leq v \leq 857 \mathrm{GHz}$. We thus neglect them.

To search for our best-fit model, we follow this scheme.

1. Take the residual map power spectra, as given in Table D.1.

2. Discard the first two bins at multipoles $\ell=53$ and 114 (following Sects. 3.2.2 and 3.2.3).

3. Correct for SZ-related residuals, $C_{\mathrm{SZ} \text { corr }}^{v \times v^{\prime}}$, and $C_{\mathrm{CIB}-\mathrm{SZcorr}}^{\nu \times v^{\prime}}$, following Eqs. (8), (9), (12), and add the errors of these corrections quadratically to the error bars given in Table D.1.

4. Apply the colour correction to convert the theoretical model from measured $\mathrm{Jy}^{2} \mathrm{sr}^{-1}$ to $\mathrm{Jy}^{2} \mathrm{sr}^{-1}\left[v I_{v}=\right.$ const.] (Eq. (38)).

5. Compute the $\chi^{2}$ value between the theoretical model and the observations, further applying the correction $C_{\mathrm{CIBcorr}}^{v \times v^{\prime}}$ (Eq. (4)), and adding calibration errors, as described in the next item.

6. The calibration uncertainties are treated differently than the CIB power spectra error bars. We use an approach similar to the galaxy number counts model of Béthermin et al. (2011). A calibration factor $f_{\text {cal }}$ is introduced. It has an initial value of 1 , but can vary inside a Gaussian prior, centred on the calibration errors given in Table 1 . We add a term to the $\chi^{2}$ that takes into account the estimated calibration uncertainties: $\chi_{\text {cal }}^{2}=\sum_{\text {bands }}\left(f_{\text {cal }}-1\right)^{2} / \sigma_{\text {cal }}^{2}$, where $\sigma_{\text {cal }}^{2}$ are the calibration errors. The $C_{\ell}$ computed for the model is thus modified according to $C_{\ell, v \times v^{\prime}}^{\mathrm{c} a l}=f_{v}^{\mathrm{cal}} f_{v^{\prime}}^{\mathrm{cal}} C_{\ell, v \times v^{\prime}}^{\text {model }}$.

\subsection{Constraints on SFRD and effective bias from the large-angle linear scales}

\subsubsection{Fitting the linear model to the data}

Planck is a unique probe of the large-scale anisotropies of the CIB. At $\ell \lesssim 1000$, the clustering is dominated by the correlation between dark matter haloes (the 2-halo term, e.g., Planck Collaboration XVIII 2011). Planck data thus give the opportunity to put new constraints on both star-formation history and clustering of star-forming galaxies, using only the linear part of the power spectra. In this modelling, we consider only the 2-halo contribution to the cross-power spectrum between maps at frequency $v$ and $v^{\prime}$ (or auto-spectrum if $v=v^{\prime}$ ), which can be written as

$C_{\ell, v, v^{\prime}}^{2 \mathrm{~h}}=\int \frac{\mathrm{d} z}{\chi^{2}} \frac{\mathrm{d} \chi}{\mathrm{d} z} a^{2} b_{\mathrm{eff}}^{2}(z) \bar{j}(v, z) \bar{j}\left(v^{\prime}, z\right) P_{\mathrm{lin}}(k=\ell / \chi, z)$,

and we fit only for $\ell \leq 600$. Here $b_{\text {eff }}$ is the effective bias of infrared galaxies at a given redshift, i.e., the mean bias of dark matter haloes hosting infrared galaxies at a given redshift weighted by their contribution to the emissivities. This term implicitly takes into account the fact that more massive haloes are more clustered. The link between this simple approach and the HOD approach of Sect. 5.5 is discussed in Appendix C. We compute $P_{\text {lin }}(k)$ using CAMB $^{7}$.

The emissivities $\bar{j}(v, z)$ are derived from the star formation density $\rho_{\mathrm{SFR}}$ following (see Appendix B)

$\bar{j}(v, z)=\frac{\rho_{\mathrm{SFR}}(z)(1+z) S_{v, \mathrm{eff}}(z) \chi^{2}(z)}{K}$,

7 http://camb.info/ where $K$ is the Kennicutt (1998) constant $\left(S F R / L_{\mathrm{IR}}=1.7 \times\right.$ $10^{-10} M_{\odot} \mathrm{yr}^{-1}$ for a Salpeter IMF) and $S_{v, \text { eff }}(z)$ the mean effective $\mathrm{SED}$ of all infrared galaxies at a given redshift. They are deduced from the Béthermin et al. (2012a) model (see Appendix B). These SEDs are a mixture of secularly star-forming galaxies and starburst galaxies. The dust temperature here increases with redshift following the measurements of Magdis et al. (2012) (see Sect. 6.4 for a discussion about the choice of the SED library for the modelling and the impact on the results).

There are degeneracies between the evolution of the bias and of the emissivities. In order to break them, we put some priors on the following quantities.

- The local infrared luminosity density, $\rho_{S F R}(z=0)=(1.95 \pm$ $0.3) \times 10^{-2} M_{\odot} \mathrm{yr}^{-1}$ (Vaccari et al. 2010), converted using the $H_{0}$ value measured by Planck).

- The local bias of infrared galaxies, $b=0.84 \pm 0.11$ (Saunders et al. 1992), converted using $\sigma_{8}$ measured by Planck.

- The mean level of the CIB deduced from galaxy number counts, $12.6_{-1.7}^{+8.3} \mathrm{nW} \mathrm{m}^{-2} \mathrm{sr}^{-1}$ at $3000 \mathrm{GHz}$ from Berta et al. (2011), 6.5 $5_{-1.6}^{+1.7} \mathrm{nW} \mathrm{m}^{-2} \mathrm{sr}^{-1}$ at $857 \mathrm{GHz}$, and $2.1_{-0.6}^{+0.7} \mathrm{nW} \mathrm{m}^{-2} \mathrm{sr}^{-1}$ at $545 \mathrm{GHz}$ from Béthermin et al. (2012c), and finally $>0.27 \mathrm{nW} \mathrm{m}^{-2} \mathrm{sr}^{-1}$ from Zemcov et al. (2010) at $353 \mathrm{GHz}$. These values are colour-corrected from PACS, SPIRE and SCUBA to Planck and IRAS, using the Béthermin et al. (2012a) model.

In this simple analysis, we want to measure only two quantities: the effective bias and its evolution with redshift, $b_{\text {eff }}(z)$; and the star formation density history, $\rho_{\mathrm{SFR}}(z)$.

Inspired by the redshift evolution of the dark matter halo bias, we chose the following simple parametric form for the evolution of the effective bias:

$b_{\text {eff }}(z)=b_{0}+b_{1} z+b_{2} z^{2}$.

For the star formation history, the values of $\rho_{\mathrm{SFR}}$ at $z=0,1,2$ and 4 are free parameters, and we connect these points assuming a power law in $(1+z)$, using the two last points to extrapolate $\rho_{\mathrm{SFR}}$ at $z>4$. We perform a Monte Carlo Markov chain analysis of the global parameter space. We assume Gaussian uncorrelated error bars for uncertainties, which are a linear combination of statistical and beam errors. The calibration uncertainties are treated following the method described Sect. 5.2.

To be independent of the exact level of the Poisson and 1-halo power spectrum in our linear analysis, we fit only for $\ell \leq$ 600 measurements. For such $\ell$ s, contamination by the Poisson and 1-halo terms is lower than $\sim 10 \%$ (except for $3000 \times 3000$ where it reaches $\sim 25 \%$ at $\ell=502$, see Fig. 10). We nevertheless add to our model the small correction due to the 1-halo and Poisson terms, as derived from the Béthermin et al. (2013) model.

\subsubsection{Results}

With a best-fit $\chi^{2}$ value of 35 for 41 degrees of freedom, we obtain a very good fit to the data. In Table 8 we quote median values and marginalized limits for the parameters. The posterior value of parameters for which we imposed a Gaussian prior (local effective bias and SFRD, plus calibration factors) are all within the $1 \sigma$ range of the prior values (except the $857 \mathrm{GHz}$ calibration factor which is at $1.2 \sigma$ ).

Figure 11 shows the evolution of the star formation density with redshift (upper panel). Our derived star formation history nicely agrees with the infrared measurements of the dustobscured star-formation rate density of Rodighiero et al. (2010) 


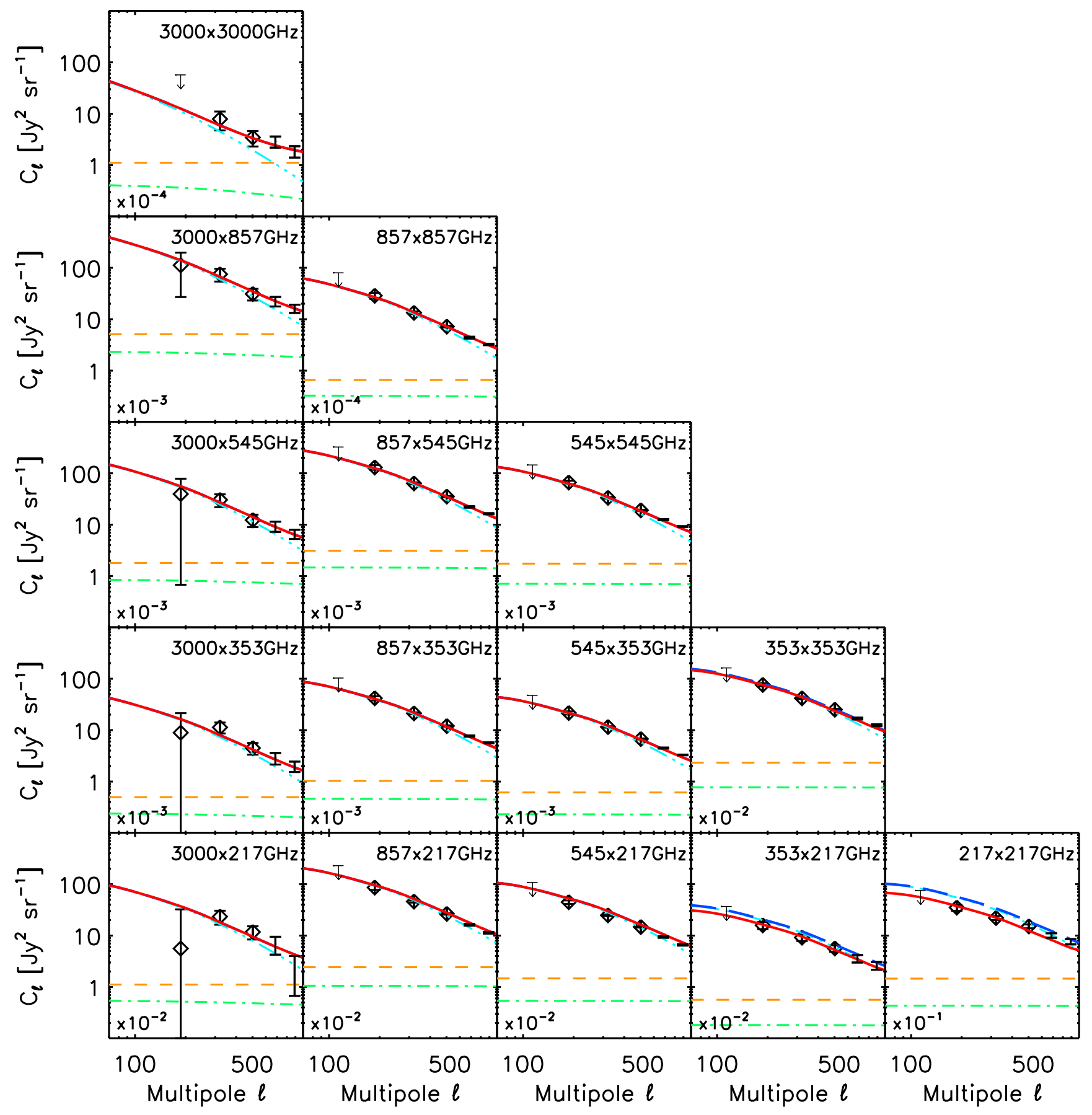

Fig. 10. (Cross-) power spectra of the CIB measured by IRAS and Planck, and the linear model. Data points are shown in black. The data used to fit the linear model are represented by diamonds $(\ell \leq 600)$. High- $\ell$ points are not displayed, as they are not used. The cyan dash-three-dot line (often lying under the red continuous line) is the best-fit CIB linear model. For completeness, we also show on this figure the shot-noise level given in Table 6 (orange dashed line) and the 1-halo term (green dot-dashed line) predicted by Béthermin et al. (2013). The red line is the sum of the linear, 1-halo and shot-noise components; it contains the spurious CIB introduced by the CMB template (see Sect. 3.1.2). The blue long-dashed line represents the CIB linear best-fit model plus 1-halo and shot-noise terms; it is corrected for the CIB leakage in the CMB map, similarly to the cyan line. When the CIB leakage is negligible, the blue long-dashed line is the same as the red continuous line.

and Magnelli et al. (2011), up to $z \sim 2$. At higher redshift, our determination is marginally compatible $(2 \sigma)$ with Gruppioni et al. (2013), but in very good agreement with the recent work of Burgarella et al. (2013) at $z=3$. We also compared our measurements with the UV estimate of star formation (not corrected for dust-attenuation) from Bouwens et al. (2007), Cucciati et al. (2012), and Reddy \& Steidel (2009). Below $z \sim 3$, the bulk of the UV light emitted by young, short-lived stars is reprocessed in the infrared. Above this redshift, we find that the star formation probed in the UV and IR regimes have roughly an equal contribution. The infrared regime alone is thus no longer a good measure of the total star-formation rate density.

We also studied the evolution of the effective bias (lower panel of Fig. 11). We measure an increase of the bias with redshift. In Fig. 11 we compare the evolution of the galaxy dark matter bias with that of dark matter haloes of various mass (from 
Table 8. Summary of the parameters of the linear model.

\begin{tabular}{llc}
\hline \hline Parameter & Definition & Median value \\
\hline$\rho_{\mathrm{SFR}}(z=0)$ & $z=0$ star form. density & $1.88_{-0.40}^{+0.44} \times 10^{-2} M_{\odot} \mathrm{yr}^{-1}$ \\
$\rho_{\mathrm{SFR}}(z=1)$ & $z=1$ star form. density $16.07_{-3.3}^{+4.6} \times 10^{-2} M_{\odot} \mathrm{yr}^{-1}$ \\
$\rho_{\mathrm{SFR}}(z=2)$ & $z=2$ star form. density $16.61_{-3.7}^{+3.4} \times 10^{-2} M_{\odot} \mathrm{yr}^{-1}$ \\
$\rho_{\mathrm{SFR}}(z=4)$ & $z=4$ star form. density & $4.0_{-1.6}^{+2.6} \times 10^{-2} M_{\odot} \mathrm{yr}^{-1}$ \\
\hline$b_{0}$ & Effective bias at $z=0$ & $0.82_{-0.10}^{+0.11}$ \\
$b_{1}$ & First order evolution & $0.34_{-0.75}^{+0.46}$ \\
$b_{2}$ & Second order evolution & $0.31_{-0.27}^{+0.43}$ \\
\hline$f_{3000}^{\text {cal }}$ & $3000 \mathrm{GHz}$ cal. factor & $1.07{ }_{-0.10}^{+0.09}$ \\
$f_{857}^{\text {cal }}$ & $857 \mathrm{GHz}$ cal. factor & $1.12_{-0.04}^{+0.04}$ \\
$f_{545}^{\text {cal }}$ & $545 \mathrm{GHz}$ cal. factor & $1.05_{-0.03}^{+0.03}$ \\
$f_{353}^{\text {cal }}$ & $353 \mathrm{GHz}$ cal. factor & $1.007_{-0.014}^{+0.014}$ \\
$f_{217}^{\text {cal }}$ & $217 \mathrm{GHz}$ cal. factor & $0.996_{-0.007}^{+0.008}$ \\
& &
\end{tabular}

Notes. The values are obtained through an MCMC analysis (median and $68 \%$ confidence limits, CL).

Tinker et al. 2008). Our results are compatible with the track of dark matter haloes with $1-310^{12} M_{\odot}$, corresponding to the halo mass of maximal efficiency of star formation, as found in recent works (e.g., Béthermin et al. 2012b; Wang et al. 2013; and Behroozi et al. 2013a; and compatible with the related lensing magnification study of Hildebrandt et al. 2013).

\subsection{Halo model for CIB anisotropies}

The halo model is a standard approach to describe the clustering of matter at all scales (Cooray \& Sheth 2002). Starting from the assumption that all galaxies live in dark matter haloes, the clustering power spectrum can be considered as the sum of two components: the 1-halo term (labelled $P_{1 \mathrm{~h}}$ ), due to correlations of galaxies within the same halo, is responsible for the small-scale clustering; while the 2-halo term $\left(P_{2 \mathrm{~h}}\right)$, sourced by galaxy correlations in different haloes, describes the large-scale clustering.

The galaxy power spectrum is completely characterized by four main ingredients: the halo bias between dark matter and haloes; the halo density profile, describing the spatial distribution of dark matter inside a given halo; the halo mass function, specifying the number density of haloes with a given mass; and a prescription for filling dark matter haloes with galaxies, the so-called Halo Occupation Distribution (HOD).

A common assumption in the simplest versions of the halo model is that all galaxies have the same luminosity, regardless of their host dark matter halo (Viero et al. 2009; Amblard et al. 2011; Planck Collaboration XVIII 2011; Xia et al. 2012; Viero et al. 2013b). However, as has already been pointed out in Shang et al. (2012), both galaxy clustering and galaxy luminosity are linked to host halo mass so that, in a statistical way, galaxies situated in more massive haloes have more stellar mass and are more luminous. The lack of such a link between galaxy luminosity

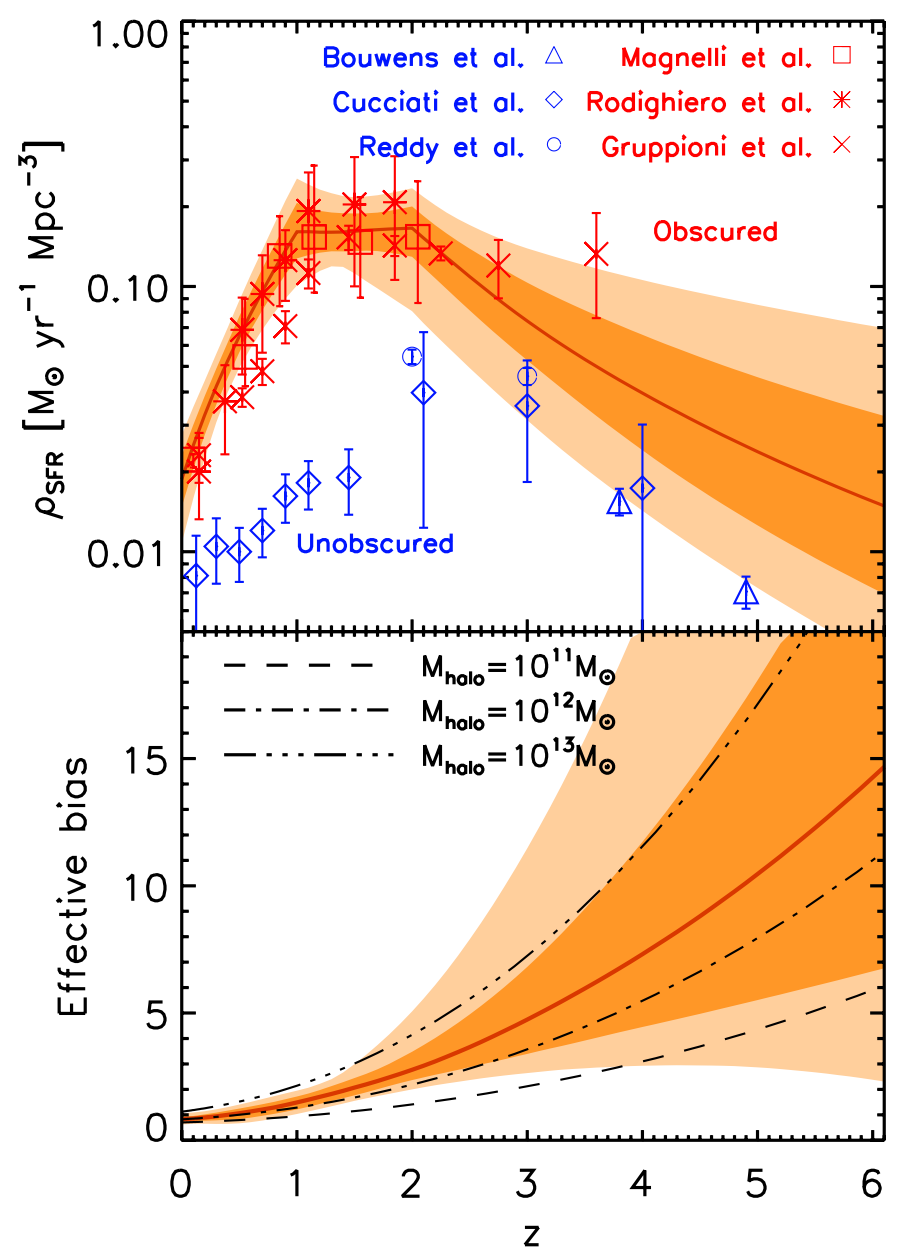

Fig. 11. Evolution of the star formation density (upper panel) and effective bias as a function of redshift (lower panel), as constrained by the linear part of the power spectra. In both panels, the median realization of the model is represented with a red line, the $\pm 1 \sigma$ confidence region with a dark orange area, and the $\pm 2 \sigma$ region with a light orange area. In the upper panel, we added the measurements of obscured star formation from infrared Magnelli et al. 2011, squares; Rodighiero et al. 2010, asterisks; Cucciati et al. 2012, diamonds; Gruppioni et al. 2013, crosses, and unobscured star formation from uncorrected UV (Bouwens et al. 2007, triangles; Reddy \& Steidel 2009, circles). In the lower panel, we also plot the evolution of the dark matter halo bias for dark matter halo mass of $10^{11} M_{\odot}$ (dashed line), $10^{12} M_{\odot}$ (dot-dashed line), and $10^{13} M_{\odot}$ (three-dots-dashed line).

and host halo mass in the model can lead to an interpretation of the clustering signal on small angular scales being due to a significant overabundance of satellite haloes (as in Amblard et al. 2011) with respect to what is found in numerical simulations (see discussion in Shang et al. 2012; Viero et al. 2013b). However, this can instead be due to a smaller number of galaxies, but with higher luminosity.

In this paper, we assume a halo model with a galaxy luminosity-halo mass relation similar to the one introduced in Shang et al. (2012) and also used in Viero et al. (2013b). We define haloes as overdense regions with a mean density equal to 200 times the mean density of the Universe and we assume an NFW profile (Navarro et al. 1997) for the halo density profile, with a concentration parameter as in Cooray \& Sheth (2002). Fitting functions of Tinker et al. (2008) and the associated prescription for the halo bias (see Tinker et al. 2010) will be used for the halo and subhalo mass functions, respectively. 
In the next subsections we will introduce the halo model that we use and we will describe how our analysis constrains its main parameters.

\subsubsection{A halo model with luminosity dependence}

The relation between the observed flux $S_{v}$ and the luminosity of a source at a comoving distance $\chi(z)$ is given by:

$S_{v}=\frac{L_{v(1+z)}}{(1+z) 4 \pi \chi^{2}(z)}$

and the galaxy emissivity $\bar{j}_{v}(z)$ can be written as

$\bar{j}_{v}(z)=\int \mathrm{d} L \frac{\mathrm{d} n}{\mathrm{~d} L}(L, z) \frac{L_{(1+z) v}}{4 \pi}$,

where $\mathrm{d} n / \mathrm{d} L$ denotes the infrared galaxy luminosity function.

In general, in order to model the galaxy luminosity-halo mass relation, we should introduce a scatter describing the probability density $P(L \mid M)$ for a halo (or a subhalo) of mass $M$ to host a galaxy with luminosity $L$ (as in the conditional luminosity function models of, e.g., Yang et al. 2003, 2005; Cooray \& Milosavljević 2005; Cooray 2006; Amblard \& Cooray 2007; and De Bernardis \& Cooray 2012). In order to keep the analysis as simple as possible, we neglect any scatter and introduce $L_{\text {cen, } v(1+z)}\left(M_{\mathrm{H}}, z\right)$ (for central galaxies) and $L_{\mathrm{sat}, v(1+z)}\left(m_{\mathrm{SH}}, z\right.$ ) (for satellite galaxies), where $M_{\mathrm{H}}$ and $m_{\mathrm{SH}}$ denote the halo and subhalo masses, respectively, Eq. (43) can be re-written as:

$$
\begin{aligned}
\bar{j}_{v}(z)= & \int \mathrm{d} M \frac{\mathrm{d} N}{\mathrm{~d} M}(z) \frac{1}{4 \pi}\left\{N_{\mathrm{cen}} L_{\mathrm{cen},(1+z) v}\left(M_{\mathrm{H}}, z\right)\right. \\
& \left.+\int \mathrm{d} m_{\mathrm{SH}} \frac{\mathrm{d} n}{\mathrm{~d} m}\left(m_{\mathrm{SH}}, z\right) L_{\mathrm{Sat},(1+z) v}\left(m_{\mathrm{SH}}, z\right)\right\},
\end{aligned}
$$

where $\mathrm{d} n / \mathrm{d} m$ denotes the subhalo mass function and $N_{\text {cen }}$ is the number of central galaxies inside a halo.

Introducing $f_{v}^{\text {cen }}$ and $f_{v}^{\text {sat }}$ for central and satellite galaxies,

$$
\begin{aligned}
f_{v}^{\mathrm{cen}}(M, z)= & N_{\mathrm{cen}} \frac{L_{\mathrm{cen},(1+z) v}\left(M_{\mathrm{H}}, z\right)}{4 \pi}, \\
f_{v}^{\mathrm{sat}}(M, z)= & \int_{M_{\min }}^{M} \mathrm{~d} m \frac{\mathrm{d} n}{\mathrm{~d} m}\left(m_{\mathrm{SH}}, z \mid M\right) \\
& \times \frac{L_{\mathrm{sat},(1+z) v}\left(m_{\mathrm{SH}}, z\right)}{4 \pi},
\end{aligned}
$$

then the power spectrum of CIB anisotropies at observed frequencies $v, v^{\prime}$ can be written as

$$
\begin{aligned}
P_{1 \mathrm{~h}, v v^{\prime}}(k, z)= & \frac{1}{\bar{j}_{v} \bar{j}_{v^{\prime}}} \int_{M_{\min }}^{\infty} \mathrm{d} M \frac{\mathrm{d} N}{\mathrm{~d} M} \\
\times & \left\{f_{v}^{\mathrm{cen}}(M, z) f_{v^{\prime}}^{\mathrm{sat}}(M, z) u(k, M, z)\right. \\
& +f_{v^{\prime}}^{\mathrm{cen}}(M, z) f_{v}^{\mathrm{sat}}(M, z) u(k, M, z) \\
& \left.+f_{v}^{\mathrm{sat}}(M, z) f_{v^{\prime}}^{\mathrm{sat}}(M, z) u^{2}(k, M, z)\right\}, \\
P_{2 \mathrm{~h}, v v^{\prime}}(k, z)= & \frac{1}{\bar{j}_{v} \bar{j}_{v^{\prime}}} D_{v}(k, z) D_{v^{\prime}}(k, z) P_{\operatorname{lin}}(k, z) .
\end{aligned}
$$

Here

$$
\begin{aligned}
D_{v}(k, z)= & \int_{M_{\min }}^{\infty} \mathrm{d} M \frac{\mathrm{d} N}{\mathrm{~d} M} b(M, z) u(k, M, z) \\
& \times\left\{f_{v}^{\mathrm{cen}}(M, z)+f_{v}^{\mathrm{sat}}(M, z)\right\},
\end{aligned}
$$

with $u(k, M, z)$ being the Fourier transform of the halo density profile.

\subsubsection{Parameterizing the $L-M$ relation}

In the simplest version of the halo model, where galaxies residing in haloes of different masses have the same luminosity, the galaxy power spectrum is fully determined by the HOD, namely the function describing the number of central and satellite galaxies in each dark matter halo. In the model used here, the power spectrum depends, additionally, on the function $L_{(1+z) v}\left(M_{\mathrm{H}}, z\right)$, where $M$ denotes the halo mass. The luminosity $L_{(1+z) v}\left(M_{\mathrm{H}}, z\right)$ depends on three variables: the redshift $z$; the mass of the host (sub)halo; and the observing frequency $v$. We will consider the following assumptions about the structure of the luminositymass relation $L(M)$.

- We assume no difference between haloes and subhaloes with the same mass, so that $L\left(M_{\mathrm{H}}, z\right)=L\left(m_{\mathrm{SH}}, z\right)$, for $M_{\mathrm{H}}=m_{\mathrm{SH}}$. While recent studies (e.g., Rodríguez-Puebla et al. 2012, 2013) show some indication that satellite galaxies tend to have slightly more stellar mass than central galaxies with the same halo mass, these results depend on the subhalo mass definition used; in particular, the luminosity-mass relation for satellites and central galaxies has been found to be not very different when the mass of the subhalo is defined at the time of accretion (as done in this paper).

- A very simple functional form (see Blain et al. 2003, and reference therein) is assumed for galaxy SEDs:

$\Theta(v, z) \propto\left\{\begin{array}{cl}v^{\beta} B_{v}\left(T_{\mathrm{d}}(z)\right) & v<v_{0} ; \\ v^{-\gamma} & v \geq v_{0} .\end{array}\right.$

Here $B_{v}$ denotes the Planck function, while the emissivity index $\beta$ gives information about the physical nature of dust and in general depends on grain composition, temperature distribution of tunnelling states and wavelength-dependent excitation (e.g., Meny et al. 2007). The power-law function is used to temper the exponential Wien tail at high frequencies and obtain a shallower SED shape, more in agreement with observations.

The temperature is assumed to be a function of redshift according to

$T_{\mathrm{d}} \equiv T_{0}(1+z)^{\alpha}$.

This dependence of the temperature with redshift can be due to different physical processes, such as more compact geometries for galaxies at high redshift (Magdis et al. 2012), a global evolution of the SED (e.g., Addison et al. 2013; Béthermin et al. 2013) or the increase of the CMB temperature with redshift (Blain 1999).

The SED functions at high and low frequencies are connected smoothly at the frequency $v_{0}$ satisfying

$$
\frac{\mathrm{d} \ln \Theta(v, z)}{\mathrm{d} \ln v}=-\gamma \text {. }
$$

The range of variation for the parameters $\alpha, \gamma$, and $v_{*}$ is large enough to ensure that we do not exclude non-negligible regions of the multidimensional parameter space; however we assume physically motivated priors for both the temperature ( $T_{0}$ in the range $20-60 \mathrm{~K}$, see measurements in e.g., Dunne et al. 2000; Chapman et al. 2005; Amblard et al. 2010; and Hwang et al. 2010) and the emissivity index ( $\beta$ in the range 1.5-2.0). The correct choice of $\beta$ is a matter of debate; measurements of Milky Way dust, and in external galaxies (e.g., Boselli et al. 2012), give values in the range 1-2, but allowing for some degree of correlation between dust temperature and emissivity index (see, e.g., Paradis et al. 2010) 
it is possible to obtain $\beta>2$ for low dust temperatures $\left(T_{\mathrm{d}} \leq 18 \mathrm{~K}\right)$. On the theoretical side, while models for both insulating and conducting materials naturally give $\beta=2$ at long wavelengths (e.g., Draine \& Lee 1984), significant deviations from the value $\beta=2$ occur when accounting for the disordered structure of the amorphous dust grains (Meny et al. 2007). Indeed, some authors (Shang et al. 2012; Viero et al. 2013b) allow for values $\beta>2$ when fitting CIB data. In this analysis we prefer to be conservative and, since we assume the condition $T_{\mathrm{d}}>20 \mathrm{~K}$, we also impose $\beta \leq 2$; this will allow us to draw solid conclusions on the other parameters of the model, avoiding regions of the parameter space whose physical interpretation is questionable.

- We assume a redshift-dependent, global normalization of the $L-M$ relation of the form

$\Phi(z)=(1+z)^{\delta}$.

The parameter $\delta$ will be allowed to vary in the range 0-7. Such a redshift dependence can be justified considering the evolution of the specific far infrared luminosity $\left(L_{\mathrm{IR}} / M_{*}\right)$ with redshift: if the ratio of stellar mass to halo mass evolves only mildly with redshift (see e.g., Neistein et al. 2011), then the ratio $L_{\mathrm{IR}} / M_{\mathrm{H}}$ should evolve approximately as the specific infrared luminosity. The semi-analytic galaxy formation model of De Lucia \& Blaizot (2007) shows the evolution of such a quantity with redshift as a power law with a slope of about 2.5, while observations performed by Oliver et al. (2010) indicate a much steeper slope, around 4.4.

- We assume a log-normal function for the dependence of the galaxy luminosity on halo mass:

$$
\Sigma(M, z)=M \frac{1}{\left(2 \pi \sigma_{L / M}^{2}\right)^{1 / 2}} \mathrm{e}^{-\left(\log _{10}(M)-\log _{10}\left(M_{\mathrm{eff}}\right)\right)^{2} / 2 \sigma_{L / M}^{2}} .
$$

Here $M_{\mathrm{eff}}$ describes the peak of the specific IR emissivity, while the parameter $\sigma_{L / M}$ describes the range of halo masses used for producing the IR luminosity; we will assume that $\sigma_{L / M}^{2}=0.5$ throughout this paper and we checked that results do not significantly change when assuming $\sigma_{L / M}^{2}=0.65$, as in Béthermin et al. (2012b).

The reason for choosing a log-normal functional form is that star formation is active only over a given range of halo masses, being suppressed at both the low- and the highmass end by mechanisms such as photoionization, supernovae heating, feedback from active galactic nuclei and virial shocks (see e.g., Benson et al. 2003; Croton et al. 2006); it is then possible to identify a peak in the $L-M$ relation, which describes the maximum in the average infrared emissivity per unit mass.

- At the low-mass end, we assume a minimum mass $M_{\min }$, which is a free parameter in the range $10^{10}-10^{11} M_{\odot}$, and we assume $L=0$ for $M<M_{\min }$.

The equation for the luminosity-mass relation can finally be written as

$L_{(1+z) v}(M, z)=L_{0} \Phi(z) \Sigma(M, z) \Theta[(1+z) v]$,

where $L_{0}$ is a free normalization parameter (which being not physically meaningful will not be further discussed).

\subsubsection{Method and data used}

In order to constrain the main parameters of our model, we fit for a total of 121 data points of the 15 possible combinations of
Planck auto- and cross-power spectra at 217, 353, 545, 857, and $3000 \mathrm{GHz}$, considering the multipole range $187 \leq \ell \leq 2649$. We use the same procedure as described in Sect. 5.3 in order to colour-correct our model to the photometric convention $v I_{v}=$ const. and to include the corrections due to CIB over-subtraction and SZ-related residuals (see points 1-6 in Sect. 5.3). There are two main differences with respect to the linear model analysis outlined above (Sect. 5.4):

- we keep all the calibration parameters fixed at $f^{\text {cal }}=1$, which assumption is justified by the analysis using the linear model, allowing us not to deal with too many parameters;

- we assume the same prior on the star formation rate density (Vaccari et al. 2010) as in the linear model but we do not use any constraints on the bias at redshift zero. We also assume flat priors on the mean level of the CIB at 545 and $857 \mathrm{GHz}$.

We perform a Monte Carlo Markov chain analysis of the global parameter space using a modification of the publicly available code CosmoMC (Lewis \& Bridle 2002). We consider variations in the following set of eight halo model parameters:

$\mathscr{P} \equiv\left\{\alpha, \beta, \gamma, \delta, M_{\mathrm{eff}}, M_{\mathrm{min}}, T_{0}, L_{0}\right\}$.

We assume the shot-noise levels given by the sum of the values quoted in Tables 6 and 7, from Béthermin et al. (2012a) and Tucci et al. (2011), respectively, and we assume flat priors around them with width given by their $1 \sigma$ error.

The total number of free parameters in our analysis is then 23 , consisting of the sum of eight halo model parameters plus 15 shot-noise parameters. While it is tempting to fix the shot-noise power spectra to their theoretically modelled values (in order not to deal with too many parameters and keep the analysis as simple as possible), we believe that, since these values are not very tightly constrained by their underlying models, it is better to let them vary as free parameters around their best estimates.

\subsubsection{Results}

With a best-fit $\chi^{2}$ of 100.7 and 98 degrees of freedom, we obtain a remarkably good fit to the data. In Table 9 we quote mean values and marginalized limits for the model parameters. In the following, we comment on the results obtained for some parameters of the model and for some derived quantities.

Peak mass $M_{\mathrm{eff}}-$ The mean value of the most efficient halo mass for generating the $\mathrm{CIB}, \log \left(M_{\mathrm{eff}} / M_{\odot}\right)=12.6 \pm 0.1$, is in good agreement with results obtained from a similar analysis using Herschel CIB data at 250, 350 and $500 \mu \mathrm{m}$ (Viero et al. 2013b), and with other analyses, using previous Planck and Herschel data (e.g., Shang et al. 2012; Xia et al. 2012), while it is slightly higher than results from other observations and simulations (e.g., Moster et al. 2010; Behroozi et al. 2013b; Béthermin et al. 2012b; Wang et al. 2013). We also checked for a possible redshift evolution of $M_{\text {eff }}$ (which can be justified in the framework of the so-called "downsizing" idea), performing an MCMC run with the functional form

$M_{\mathrm{eff}}=M_{0} \times(1+z)^{q}$.

The large degeneracy between $M_{0}$ and $q$ leads to very high values of $M_{0}$. The bias and SFRD have the same redshift evolution as in the case $q=0$, but with much larger error bars (they are a factor of 6 times higher for the bias, for example). 
A\&A 571, A30 (2014)

Table 9. Mean values and marginalized 68\% CL for halo model parameters and shot-noise levels (in $\mathrm{Jy}^{2} \mathrm{sr}^{-1}$ ).

\begin{tabular}{|c|c|c|}
\hline Parameter & Definition & Mean value \\
\hline $\bar{\alpha}$ & SED: redshift evolution of the dust temperature & $0.36 \pm 0.05$ \\
\hline$T_{0}[\mathrm{~K}]$ & SED: dust temperature at $z=0$ & $24.4 \pm 1.9$ \\
\hline$\beta$ & SED: emissivity index at low frequency & $1.75 \pm 0.06$ \\
\hline$\gamma$ & SED: frequency power law index at high frequency & $1.7 \pm 0.2$ \\
\hline$\delta$ & Redshift evolution of the normalization of the $L-M$ relation & $3.6 \pm 0.2$ \\
\hline $\log \left(M_{\mathrm{eff}} / M_{\odot}\right)$ & Halo model most efficient mass & $12.6 \pm 0.1$ \\
\hline$M_{\min }\left[M_{\odot}\right]$ & Minimum halo mass & unconstrained \\
\hline$S^{3000 \times 3000}$ & Shot noise for $3000 \mathrm{GHz} \times 3000 \mathrm{GHz}$ & $9585 \pm 1090$ \\
\hline$S^{3000 \times 857}$ & Shot noise for $3000 \mathrm{GHz} \times 857 \mathrm{GHz}$ & $4158 \pm 443$ \\
\hline$S^{3000 \times 545}$ & Shot noise for $3000 \mathrm{GHz} \times 545 \mathrm{GHz}$ & $1449 \pm 176$ \\
\hline$S^{3000 \times 353}$ & Shot noise for $3000 \mathrm{GHz} \times 353 \mathrm{GHz}$ & $411 \pm 48$ \\
\hline$S^{3000 \times 217}$ & Shot noise for $3000 \mathrm{GHz} \times 217 \mathrm{GHz}$ & $95 \pm 11$ \\
\hline$S^{857 \times 857}$ & Shot noise for $857 \mathrm{GHz} \times 857 \mathrm{GHz}$ & $5364 \pm 343$ \\
\hline$S^{857 \times 545}$ & Shot noise for $857 \mathrm{GHz} \times 545 \mathrm{GHz}$ & $2702 \pm 124$ \\
\hline$S^{857 \times 353}$ & Shot noise for $857 \mathrm{GHz} \times 353 \mathrm{GHz}$ & $953 \pm 54$ \\
\hline$S^{857 \times 217}$ & Shot noise for $857 \mathrm{GHz} \times 217 \mathrm{GHz}$ & $181 \pm 6$ \\
\hline$S^{545 \times 545}$ & Shot noise for $545 \mathrm{GHz} \times 545 \mathrm{GHz}$ & $1690 \pm 45$ \\
\hline$S^{545 \times 353}$ & Shot noise for $545 \mathrm{GHz} \times 353 \mathrm{GHz}$ & $626 \pm 19$ \\
\hline$S^{545 \times 217}$ & Shot noise for $545 \mathrm{GHz} \times 217 \mathrm{GHz}$ & $121 \pm 6$ \\
\hline$S^{353 \times 353}$ & Shot noise for $353 \mathrm{GHz} \times 353 \mathrm{GHz}$ & $262 \pm 8$ \\
\hline$S^{353 \times 217}$ & Shot noise for $353 \mathrm{GHz} \times 217 \mathrm{GHz}$ & $54 \pm 3$ \\
\hline$S^{217 \times 217}$ & Shot noise for $217 \mathrm{GHz} \times 217 \mathrm{GHz}$ & $21 \pm 2$ \\
\hline
\end{tabular}

Constraints on the dust temperature - Parameterizing the average dust temperature of sources as

$T_{\mathrm{d}}(z)=T_{0}(1+z)^{\alpha}$,

the data suggest a redshift evolution of the temperature, with $T_{0}=(24.4 \pm 1.9) \mathrm{K}$ and $\alpha=0.36 \pm 0.05$. Such a trend, implying some kind of SED evolution, has been also found in e.g., Addison et al. (2013); Viero et al. (2013b). Experimental results from different surveys appear to have been quite contradictory, with systematics playing a critical role (e.g., Chapman et al. 2005; Coppin et al. 2008; Pascale et al. 2009; Amblard et al. 2010; Hwang et al. 2010; Chapin et al. 2011). But recently, some consensus has emerged on a scenario with an increase of dust temperature with redshift (Magdis et al. 2012; Viero et al. 2013a). The increase of temperature may be explained by a harder interstellar radiation field at earlier times (see Magdis et al. 2012, for a detailed discussion).

Constraints on the bias - Galaxies are considered as a biased tracer of the dark matter field. The galaxy overdensity $\delta_{\mathrm{g}}(k, z)$ is assumed to trace the underlying dark matter field $\delta_{\mathrm{dm}}(k, z)$ via

$\delta_{\mathrm{g}}(k, z)=b(k, z) \delta_{\mathrm{dm}}(k, z)$,

where $b(k, z)$ is the galaxy bias, which in general can depend not only on scale and redshift but also on luminosity, spectral type and colour. On large scales, the bias is generally assumed to be scale-independent; however, both numerical simulations (Kauffmann et al. 1999) and recent results from galaxy-galaxy lensing and galaxy clustering also indicate an increase of the bias with redshift (e.g., Mandelbaum et al. 2013), while Tegmark \& Peebles (1998) show that the bias must be close to unity when approaching $z=0$. The combination of CMB and large-scale clustering data yields a bias parameter $b \sim 1$ (Verde et al. 2002) while Saunders et al. (1992) found $b \sigma_{8}=0.84 \pm 0.11$ for IRAS galaxies, which, assuming $\sigma_{8}=0.8$, gives $b \sim 0.86$. In Fig. 11 we show our estimate of the redshift dependent bias; it is remarkable that, without assuming any prior on the value of the bias at redshift zero, we are able to obtain a very good fit to observations, with $b(z=0)=1.1 \pm 0.02$.
Constraints on star formation history - The mean value and $68 \%$ CL bounds on the cosmic star formation rate density $\rho_{\mathrm{SFR}}$ are plotted in Fig. 11. The parameter $\rho_{\mathrm{SFR}}$ has been computed following Eq. (40), replacing $s_{v, \text { eff }}(z)$ by the halo model SED as given in Eq. (50). We use the Kennicutt (1998) constant to convert infrared luminosity to star formation rate $\left(S F R / L_{\mathrm{IR}}=\right.$ $1.7 \times 10^{-10} M_{\odot} \mathrm{yr}^{-1}$ for a Salpeter IMF). As can be seen from Fig. 11, the star formation rate densities predicted by both models used in this paper are in very good agreement for redshifts $z \lesssim 2$ while there is a significant difference at higher redshifts. Interestingly, Behroozi et al. (2013b) reports a compilation of results, showing how measurements performed before 2006 predict quite a high value for the high redshift SFRD (then more compatible with the halo model results, see also the compilation in Hopkins \& Beacom 2006), while measurements performed after 2006 and obtained with different assumptions about the dust present at $z>3$, show a rapid decrease of the SFRD, which then becomes more compatible with results obtained using the linear model. In an attempt to reproduce the break in the SFRD seen in Fig. 14 for the linear model, we also imposed the condition $\delta=0$ at redshift $z=2$ in the redshift normalization parameter $\Phi(z)$ of the $L-M$ relation. Such a condition has already been considered in Shang et al. (2012) and, while it is motivated by some observations, it is also hard to explain from a theoretical point of view (e.g., Weinmann et al. 2011). In this case, we are able to obtain lower values of the SFRD at high redshifts (see Fig. 13), but at the price of slightly degrading the quality of the fit. We finally note that another potential reason for the discrepancy found at high redshift can be the difference in the inferred bias evolution between the linear model and the halo model: in fact, a lower value of the bias at high redshift (as found in the context of the halo model) can be compensated by higher values for the SFRD in the same redshift range. We will come back to the SFRD discussion in Sect. 6.4.

Finally, we are also able to determine the mean CIB intensity at the Planck frequencies considered in the analysis. The values obtained are presented in Table 10. Although higher, they are compatible, within the $95 \% \mathrm{CL}$, with results obtained from number counts measurements (Béthermin et al. 2012c). 


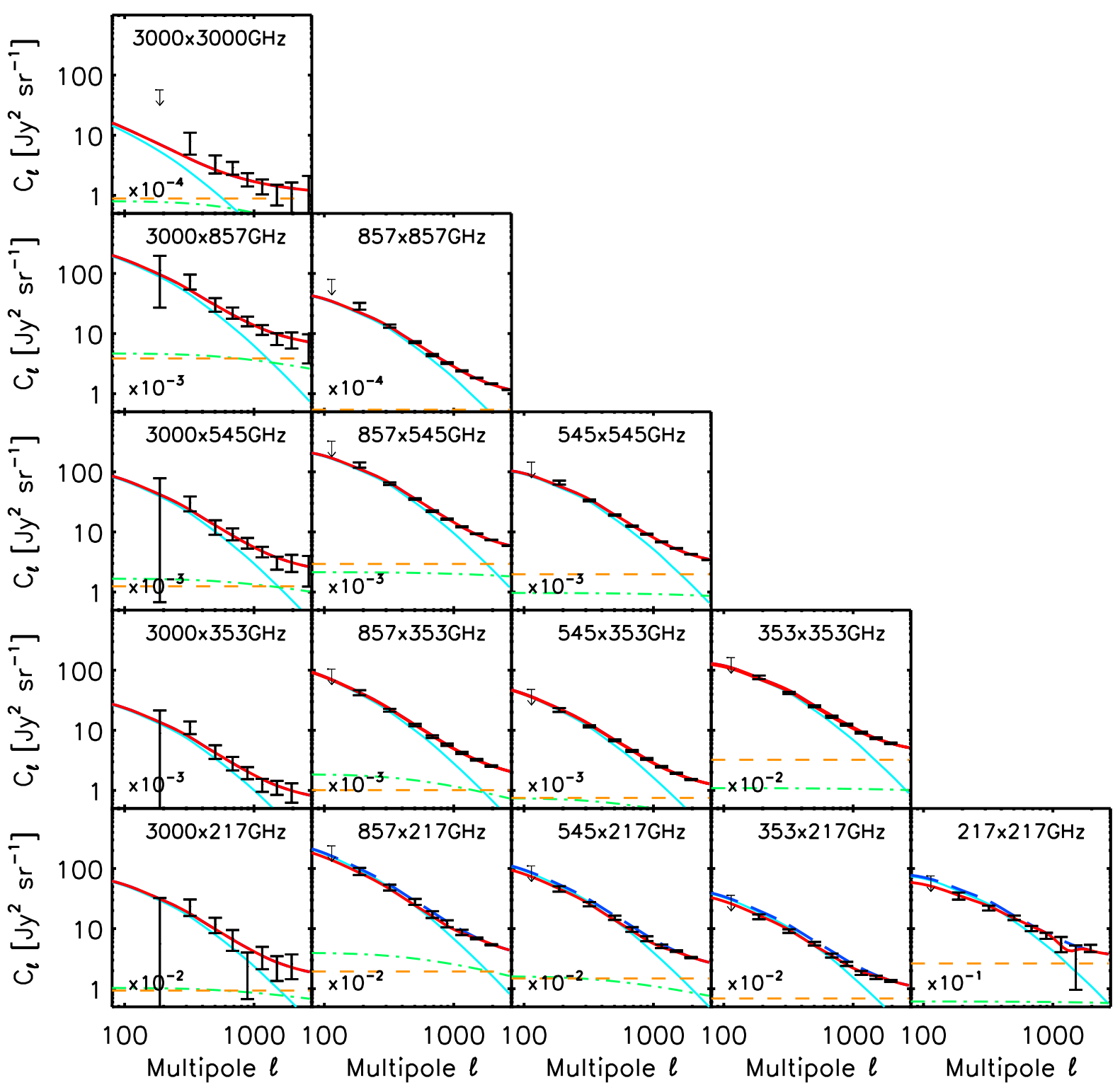

Fig. 12. (Cross-) power spectra of the CIB anisotropies measured by Planck and IRAS, compared with the best-fit extended halo model. Data points are shown in black. The red line is the sum of the linear, 1-halo and shot-noise components, which is fitted to the data. It contains the spurious CIB introduced by the CMB template (see Sect. 3.1.2). The orange dashed, green dot-dashed, and cyan three-dots-dashed lines are the best-fit shot-noise level, the 1-halo and the 2-halo terms, respectively. They are corrected for the CIB leakage in the CMB. The sum of the three is the blue long-dashed line. When the CIB leakage is negligible, the blue long-dashed line is the same as the red continuous line.

\subsection{CIB-CMB lensing cross-correlation}

We tested the validity of our approach by comparing the predictions for our best-fit models with the measurements of the cross-correlation between the CIB and the CMB lensing potential presented in Planck Collaboration XVIII (2014). For the linear model, we computed the cross-correlation following:

$C_{\ell}^{v \phi}=\int b_{\mathrm{eff}}(z) \bar{j}(v, z) \frac{3}{\ell^{2}} \Omega_{r m m}, H_{0}^{2}\left(\frac{\chi_{*}-\chi}{\chi_{*} \chi}\right) P_{\operatorname{lin}}(k=\ell / \chi, z) \mathrm{d} \chi$,

where $\chi_{*}$ is the comoving distance to the CMB last-scattering surface. We use a similar equation for the extended halo model.

Figure 15 shows a comparison between the model and the data. The halo model (as well as its variant with a break in the temperature and global normalization of the $L-M$ relation
Table 10. Derived estimates of the CIB intensity from the extended halo model.

\begin{tabular}{lc}
\hline \hline Band & $v I_{v}\left[\mathrm{nW} \mathrm{m}^{-2} \mathrm{sr}^{-1}\right]$ \\
\hline $3000 \mathrm{GHz}$ & $13.1 \pm 1.0$ \\
$857 \mathrm{GHz}$ & $7.7 \pm 0.2$ \\
$545 \mathrm{GHz}$ & $2.3 \pm 0.1$ \\
$353 \mathrm{GHz}$ & $0.53 \pm 0.02$ \\
$217 \mathrm{GHz}$ & $0.077 \pm 0.003$ \\
\hline
\end{tabular}

at redshift $z \sim 4$, see Sect. 6.4) agrees remarkably well with the measurements for all channels. The linear model gives a higher prediction at 217 and $353 \mathrm{GHz}$ (although compatible with the data points at the $1 \sigma$ level). 


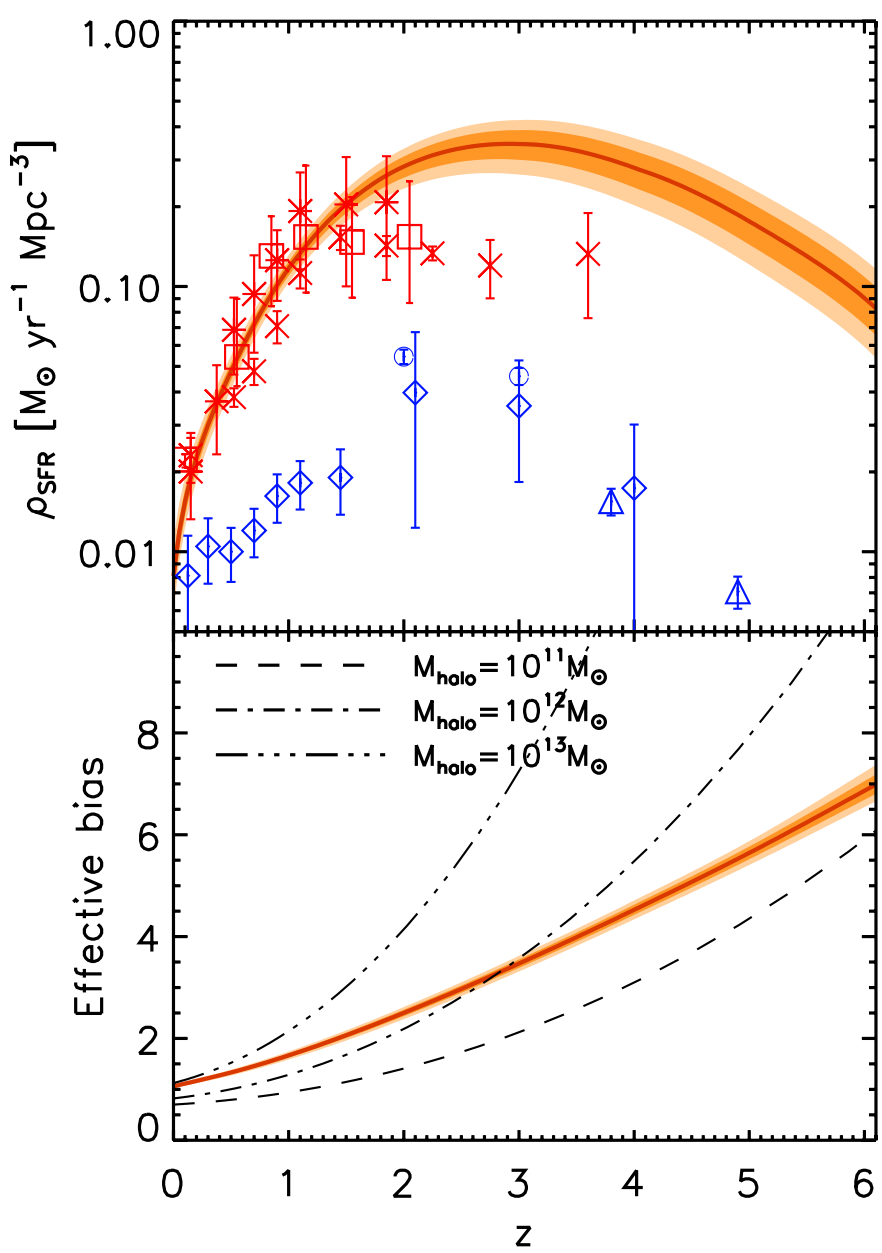

Fig. 13. Evolution of the star formation density (upper panel) and effective bias as a function of redshift (lower panel), as constrained from our extended halo model. In both panels, the median realization of the model is represented with a red line, the $\pm 1 \sigma$ confidence region in dark orange, and the $\pm 2 \sigma$ region in light orange. In the upper panel the reported data are the same as in Fig. 11. In the lower panel, we also plot the evolution of the dark matter-halo bias for dark matter halo masses of $10^{11} M_{\odot}$ (dashed line), $10^{12} M_{\odot}$ (dot-dashed line), and $10^{13} M_{\odot}$ (threedots-dashed line).

\section{Discussion}

\subsection{The $143 \mathrm{GHz}$ case}

Removing the CMB anisotropy at $143 \mathrm{GHz}$ is very problematic, since the CMB power spectrum is about 5000 higher than the CIB at $\ell=100$. However, thanks to the exceptional quality of the Planck data, and the accuracy of the 100 and $143 \mathrm{GHz}$ relative photometric calibration, we can obtain interesting measurements, using the same method to clean the maps and measure the power spectra as for the other channels. We show the measurements in Fig. 16, together with the best-fit CIB model. This estimate has been obtained by correcting the measurements for the SZ and spurious CIB (induced by the use of the $100 \mathrm{GHz}$ map as a CMB template). Those corrections are important, especially for power spectra at low frequencies. For example, for $143 \times$ 217, the SZ-related corrections decrease the measurements by 10-20\%, while the correction for the spurious CIB (including the shot noise) increases the measurements by $30-60 \%$. Since these corrections are large, we have not attempted to include the $143 \mathrm{GHz}$ measurements when constraining the model, and we recommend the reader to take them with caution.

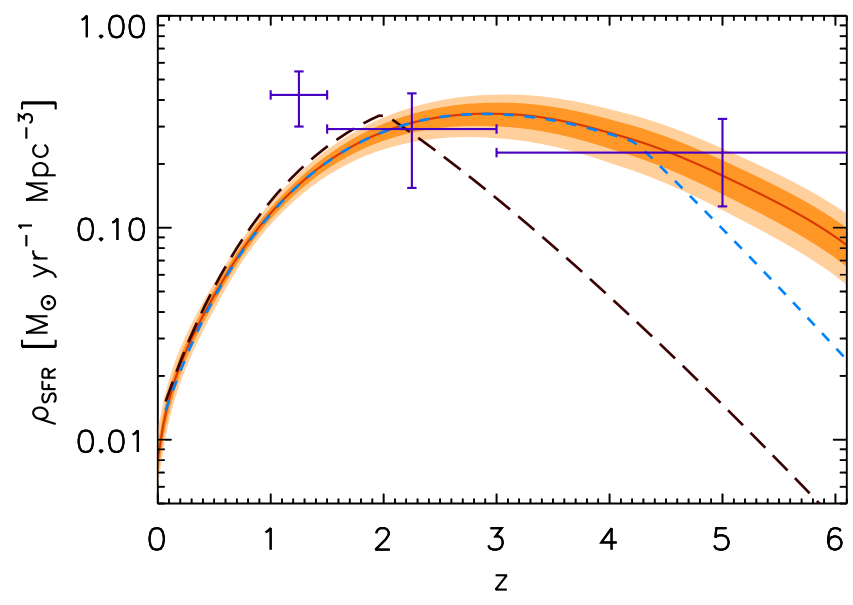

Fig. 14. Marginalized constraints on the star formation rate density, as derived from our extended halo model described in Sect. 5.5 (red continuous line with \pm 1 and $\pm 2 \sigma$ orange dashed areas). It is compared with mean values computed imposing the condition $\delta(z \geq 2)=0$ (black long-dashed line), or the combined conditions $\delta\left(z \geq z_{\text {break }}\right)=0$ and $T\left(z=z_{\text {break }}\right)=T\left(z_{\text {break }}\right)$, where $z_{\text {break }}$ is found to be $4.2 \pm 0.5$ (blue dashed line). The violet points with error bars are the SFR density determined from the modelling of the CIB-CMB Lensing cross correlation by Planck Collaboration XVIII (2014).

We show in Fig. 16 a comparison between extrapolation of the halo best-fit model to the $143 \mathrm{GHz}$ cross-power spectra and our CIB power-spectrum estimates. The $143 \times v$ cross-power spectra agree quite well for $\ell<1000$, at least for $v \geq 353 \mathrm{GHz}$. The $143 \times 217 \mathrm{CIB}$ power spectrum lies about $2 \sigma$ above the prediction at intermediate scales $(\ell=502$ and 684). This CIB overestimate increases for the $143 \times 143$ power spectrum, which is certainly the most difficult to obtain; this is in excess with respect to the prediction for $300<\ell<1000$. At this frequency, however, the CIB auto-power spectrum measurements have to be taken with caution, as the correction for the spurious CIB can be as high as $70 \%$, and is thus highly model dependent.

\subsection{Frequency decorrelation}

Using the power spectrum measurements, we can quantify the frequency decoherence. We measure the correlation between bands by averaging the quantity $C_{\ell}^{\nu \times v^{\prime}} /\left(C_{\ell}^{\nu \times v} \times C_{\ell}^{\nu^{\prime} \times v^{\prime}}\right)^{1 / 2}$ for $150<\ell<1000$. We restrict ourselves to this $\ell$ range to have only the clustered CIB contribution (not the shot noise). Results are given in Table 11. We see that the CIB for the four HFI frequencies (from 217 to $857 \mathrm{GHz}$ ) is very well correlated, the worst case being between the 857 and $217 \mathrm{GHz}$ channels, with a correlation of about 0.85 . On the other hand, the correlation of all HFI bands with IRIS is quite low, between about 0.2 and 0.32 (with a large dispersion). This is expected, because the redshift distribution of CIB anisotropies evolves strongly between 3000 and $\leq 857 \mathrm{GHz}$, being biased towards higher redshifts at lower frequencies (e.g., Béthermin et al. 2013).

Contrary to the range $217-857 \mathrm{GHz}$ the band correlation strongly varies with $\ell$ at $143 \mathrm{GHz}$, decreasing from $\ell=150$ to 1000 (see Table 11). Such a decrease is expected, based on the high shot-noise contribution at this frequency (see Fig. 16), and the fact that the shot noise is dominated by the radio source contribution, contrary to the $\geq 217 \mathrm{GHz}$ channels. Moreover, for dusty star-forming galaxies, Béthermin et al. (2013) observed that the band correlation is lower for the shot noise than for 
Planck Collaboration: Planck 2013 results. XXX.

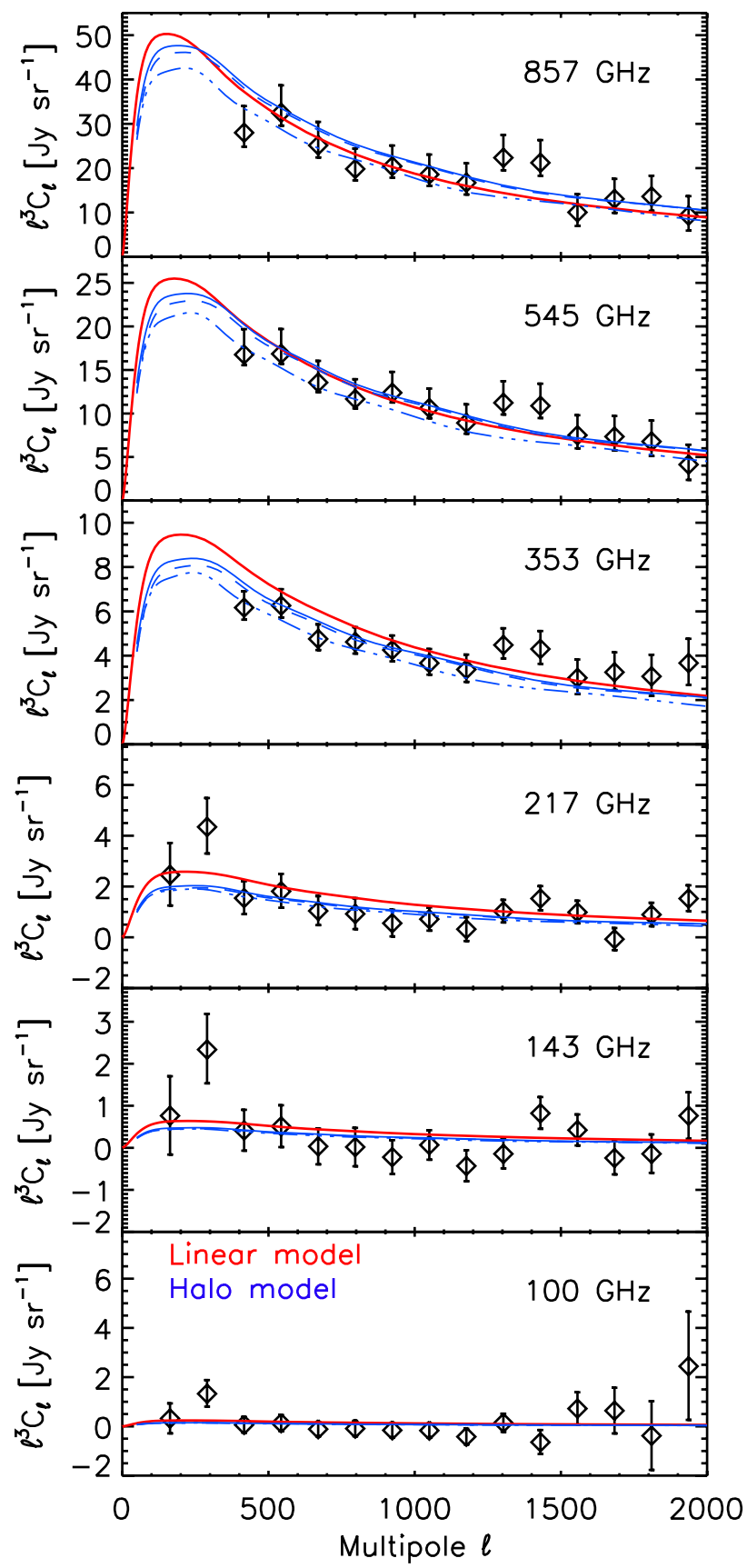

Fig. 15. Comparison between the measurements of the CIB and gravitational potential cross-correlation given in Planck Collaboration XVIII (2014) (diamonds), with the predictions from our best-fit models of the CIB cross-power spectra (red and blue solid lines for the linear and extended halo model, respectively). The other curves are the two variants of the extended halo model with: (i) a break in the global normalization of the $L-M$ relation fixed at redshift $z=2$ (blue 3-dot-dashed curve); and (ii) a break in both the temperature evolution and normalization of the $L-M$ relation, found at redshift $z=4.2 \pm 0.5$ (blue long-dashed curve).

correlated anisotropies; this also mimics a scale dependence when the shot-noise contribution is important.

\subsection{Comparison with recent measurements}

We now compare the CIB auto-spectrum measurements with the most recent measurements from Herschel-SPIRE

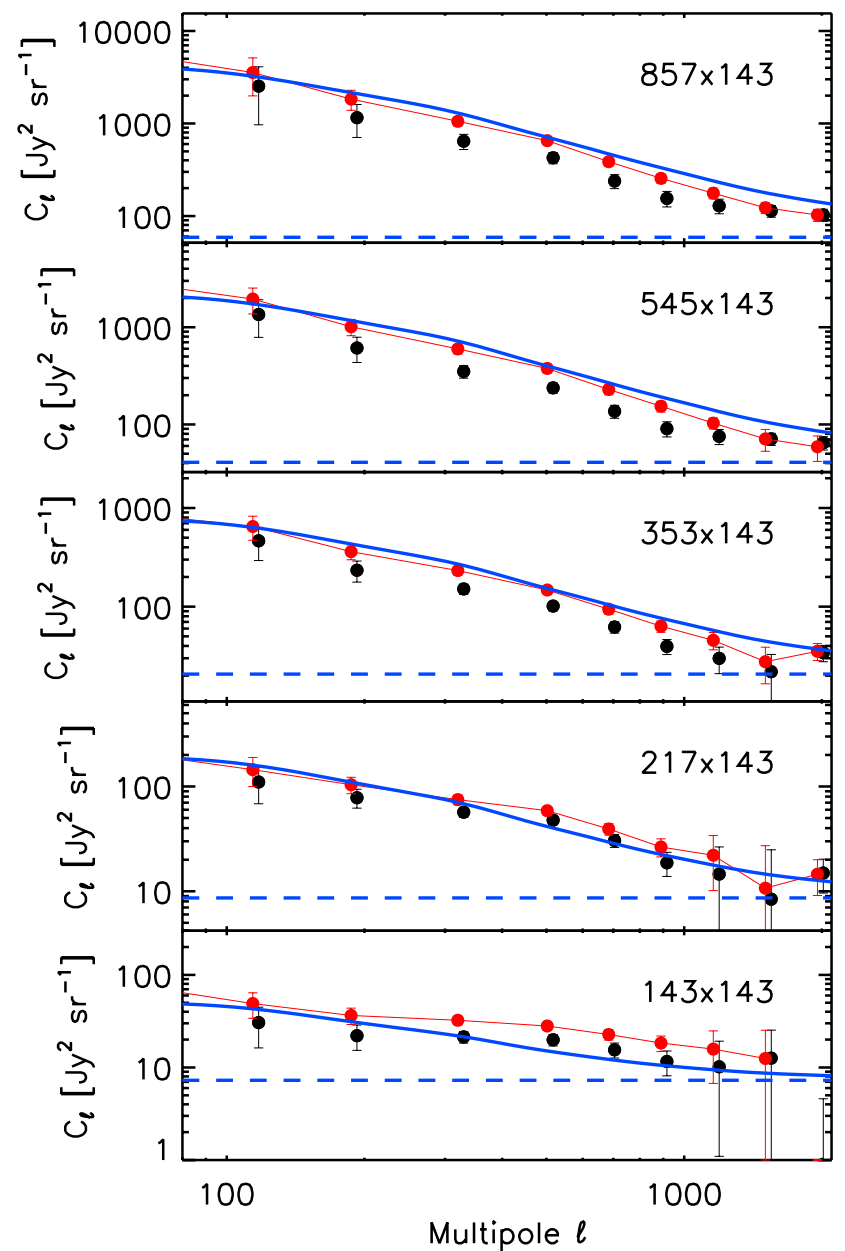

Fig. 16. CIB cross- and auto- power spectra obtained at $143 \mathrm{GHz}$ (red points). To obtain the CIB, the cleaned CMB and Galactic dust power spectra (black points, shifted in $\ell$ for clarity) are corrected for SZ-related residuals, $C_{\text {SZcorr }}^{v \times v^{\prime}}$ and $C_{\text {CIB-SZcorr }}^{\nu \times v^{\prime}}$ (following Eqs. (8), (9), and (12)), and for $C_{\mathrm{CIBcorr}}^{\nu \times v^{\prime}}$ (following Eq. (4), and computed using the extrapolation of the best-fit halo model). The prediction of the halo model is shown in blue (continuous for $2 \mathrm{~h}+1 \mathrm{~h}+$ shot noise; dashed for shot noise only).

(Viero et al. 2013b) and the earlier measurements from Planck (Planck Collaboration XVIII 2011). We compute the SPIREHFI colour corrections using the CIB SED from Gispert et al. (2000) and the most recent bandpasses (see Planck Collaboration IX 2014). In order to compare with HFI at 857 and $545 \mathrm{GHz}$, the power spectra at 350 and $500 \mu \mathrm{m}$ have to be multiplied by 1.016 and 0.805 , respectively. We use the SPIRE power spectra with only extended sources masked (such that the Poisson contribution is the same in both measurements). We see from Fig. 17 that the agreement between the SPIRE and HFI measurements (red circles versus blue circles) is excellent at $857 \mathrm{GHz}$. At $545 \mathrm{GHz}$, although compatible within the error bars there is a small difference, with the SPIRE power spectrum being higher than HFI by about $7 \%$ for $650<\ell<1800$ and by about $30 \%$ for $200<\ell<600$.

Between the publication of Planck Collaboration XVIII (2011) and this paper, the photometric calibration of the two high-frequency HFI channels has been modified (see Planck Collaboration VIII 2014). Using a planet-based calibration rather than a FIRAS-based calibration leads to a division of the calibration factors by 1.07 and 1.15 at 857 and $545 \mathrm{GHz}$, 
Table 11. Frequency decoherence of the CIB, measured by averaging $C_{\ell}^{v v^{\prime}} /\left(C_{\ell}^{\gamma v} \times C_{\ell}^{\nu^{\prime} v^{\prime}}\right)^{1 / 2}$ for $150<\ell<1000$.

\begin{tabular}{rcccccc}
\hline \hline & 3000 & 857 & 545 & 353 & 217 & 143 \\
\hline 3000 & 1 & $0.36 \pm 0.06$ & $0.31 \pm 0.04$ & $0.29 \pm 0.04$ & $0.25 \pm 0.07$ & $\ldots$ \\
857 & $\ldots$ & 1 & $0.949 \pm 0.005$ & $0.911 \pm 0.003$ & $0.85 \pm 0.05$ & $0.45 \pm 0.10[0.33-0.57]$ \\
545 & $\ldots$ & $\ldots$ & 1 & $0.983 \pm 0.007$ & $0.90 \pm 0.05$ & $0.51 \pm 0.11[0.37-0.65]$ \\
353 & $\ldots$ & $\ldots$ & $\ldots$ & 1 & $0.91 \pm 0.05$ & $0.54 \pm 0.11[0.41-0.68]$ \\
217 & $\ldots$ & $\ldots$ & $\ldots$ & $\ldots$ & 1 & $0.78 \pm 0.08[0.66-0.84]$ \\
143 & $\ldots$ & $\ldots$ & $\ldots$ & $\ldots$ & $\ldots$ & 1 \\
\hline
\end{tabular}

Notes. The error bars correspond to the standard deviation. The values at $143 \mathrm{GHz}$ strongly depend on the correction of the spurious CIB (that has been introduced by the choice of our CMB template), which is highly model dependent. The band correlation is strongly varying with $\ell$ at $143 \mathrm{GHz}$, increasing as one goes from $\ell=1000$ to 150 . The numbers in brackets at $143 \mathrm{GHz}$ indicate this variation.

respectively. After correcting for these factors, the two Planck CIB measurements agree within $1 \sigma$ at $545 \mathrm{GHz}$ and within $2 \sigma$ at $857 \mathrm{GHz}$. At $217 \mathrm{GHz}$, the discrepancy we observe between the earlier Planck CIB measurements and those presented in this paper is explained by the SZ and CIB contamination of the $143 \mathrm{GHz}$-based CMB template used in Planck Collaboration XVIII (2011). Note that with the new measurements, we do not improve the error bars, since the use of the $100 \mathrm{GHz}$ channel as a CMB template adds more noise than the use of the $143 \mathrm{GHz}$ channel. The apparent difference in shape between the CIB at $217 \mathrm{GHz}$ and at higher frequencies has to be attributed to the shot noise, whose contribution relative to the correlated part is higher at $217 \mathrm{GHz}$, making the measured CIB flatter at this frequency.

For cross-power spectra, we can compare our determination with Hajian et al. (2012). We show in Fig. 18 the $857 \times 217$ and $857 \times 143$ Planck power spectra, and those obtained from the cross-correlation between BLAST and ACT data. For this comparison, the shot noise contributions have been removed, as they are very different for BLAST, ACT and Planck. We have not applied any colour correction. Even if it appears that three points overlap in scale, the comparison can only be done at one of them, since the low $\ell$ measurements from BLAST $\times$ ACT are very noisy. For this $\ell=1750$ scale, the two measurements agree within $1 \sigma$. This plot illustrates the complementarity between Planck and the high- $\ell$ measurements from ACT and SPT - interesting constraints will come from combining the various $\ell$ ranges and frequencies probed. However, a full analysis will require careful consideration of the different instrumental bandpasses, as well as the treatment of foregrounds and point source masking. At a first look, we can compare the CIB band-powers in the three experiments by extrapolating our best-fit model up to $\ell=3000$. We obtain $\mathcal{D}_{\ell}=\ell(\ell+1) C_{\ell} / 2 \pi=63.8$ and $5.9 \mu \mathrm{K}_{\mathrm{CMB}}^{2}$ at 217 and $143 \mathrm{GHz}$, respectively. These numbers have to be compared with $\mathcal{D}_{\ell}=(54 \pm 16)$ and $(4.8 \pm 0.9) \mu \mathrm{K}_{\mathrm{CMB}}^{2}$ for ACT at 219.6 and 149.7 GHz, respectively (Dunkley et al. 2013); and $\mathcal{D}_{\ell}=(56 \pm 4)$ and $(6.2 \pm 0.5) \mu \mathrm{K}_{\mathrm{CMB}}^{2}$ for SPT at 219.6 and $153.8 \mathrm{GHz}$, respectively (Reichardt et al. 2012).

\subsection{The history of star formation density}

The star formation histories recovered from the two different modelling approaches presented in Sects. 5.4 and 5.5 are consistent below $z=2$, and agree with recent estimates of the obscured star-formation density measured by Spitzer and Herschel. At higher redshift, there are discrepancies between our two models and the estimate of Gruppioni et al. (2013). The linear model is about 1 and $2 \sigma$ lower than their measurements at $z=2.5$ and 3.5 , respectively, while the halo model lies about $>3 \sigma$ above these data points. Such estimates assume a shape for the infrared luminosity function. They are strongly dependent on the faintend slope assumption, since no data are available below the break of the luminosity function. This shows how measurement of the obscured star formation rate density at $z>3$ is difficult.

We investigated the origin of the discrepancy between the two modelling approaches. In particular, we modified the halo model in two ways to see how different assumptions on the parametrization of the model can affect the results.

1. We fit the data by imposing the condition $\delta=0$ for redshifts $z \geq z_{\text {break }}=2$ in the redshift normalization parameter $\Phi(z)$ of the luminosity-mass relation. This parametrization, although degrading the quality of the fit somewhat, decreases the SFRD by a factor of about 5 for $z=4$, which is now compatible with the linear model SFRD (see Fig. 13).

2. We fit the data by imposing two conditions: $\delta=0$ for redshift $z \geq z_{\text {break }}$ and $T\left(z \geq z_{\text {break }}\right)=$ constant $=T\left(z_{\text {break }}\right)$ for $z_{\text {break }}$ in the range $2-5$. We find $z_{\text {break }}=4.2 \pm 0.5$, as can be seen in Fig. 13. In this case the SFRD is only reduced at very high redshift, by a factor 3 at $z=6$. Note that allowing for a redshift break in the redshift evolution of the temperature avoids reaching unphysically high values at very high redshift.

We also show in Fig. 13 the SFRD measurements from the CIB-CMB lensing cross-correlation (Planck Collaboration XVIII 2014). This compares favourably with a high SFRD level at high redshift.

Part of the discrepancy between the two modelling approaches can also be attributed to the effective bias. A higher bias, as that recovered at high redshift from the linear model, favours a lower SFRD.

We finally compared the SEDs used in the two approaches. The effective SEDs present a broader peak than the extended halo model SEDs, because they take into account the dispersion in dust temperature and the mixing between secularly starforming galaxies and episodic starbursts. At $z>2$, there are discrepancies in the Rayleigh-Jeans regime between the two templates, the effective SEDs being higher than the extended halo model SEDs; this discrepancy increases with redshift. At $z=5$, for the same $L_{\mathrm{IR}}$, the parametric SEDs of the halo model emits about 3 times less infrared light than the effective SEDs (and hence about an order of magnitude less fluctuations). This explains why this model requires a much higher star formation rate density to fit CIB anisotropies than the linear model ${ }^{8}$. Following the parametrization of Eq. (50), we fit the effective SEDs with a modified black body with a $v^{1.75}$ emissivity law to obtain the dust temperature. In Fig. 19, we show the redshift evolution of the temperature of the two templates. Compared to

\footnotetext{
8 We obtain the same SFRD if we fix in the linear model the SEDs and effective bias to those obtained from the extended halo model.
} 
Planck Collaboration: Planck 2013 results. XXX.

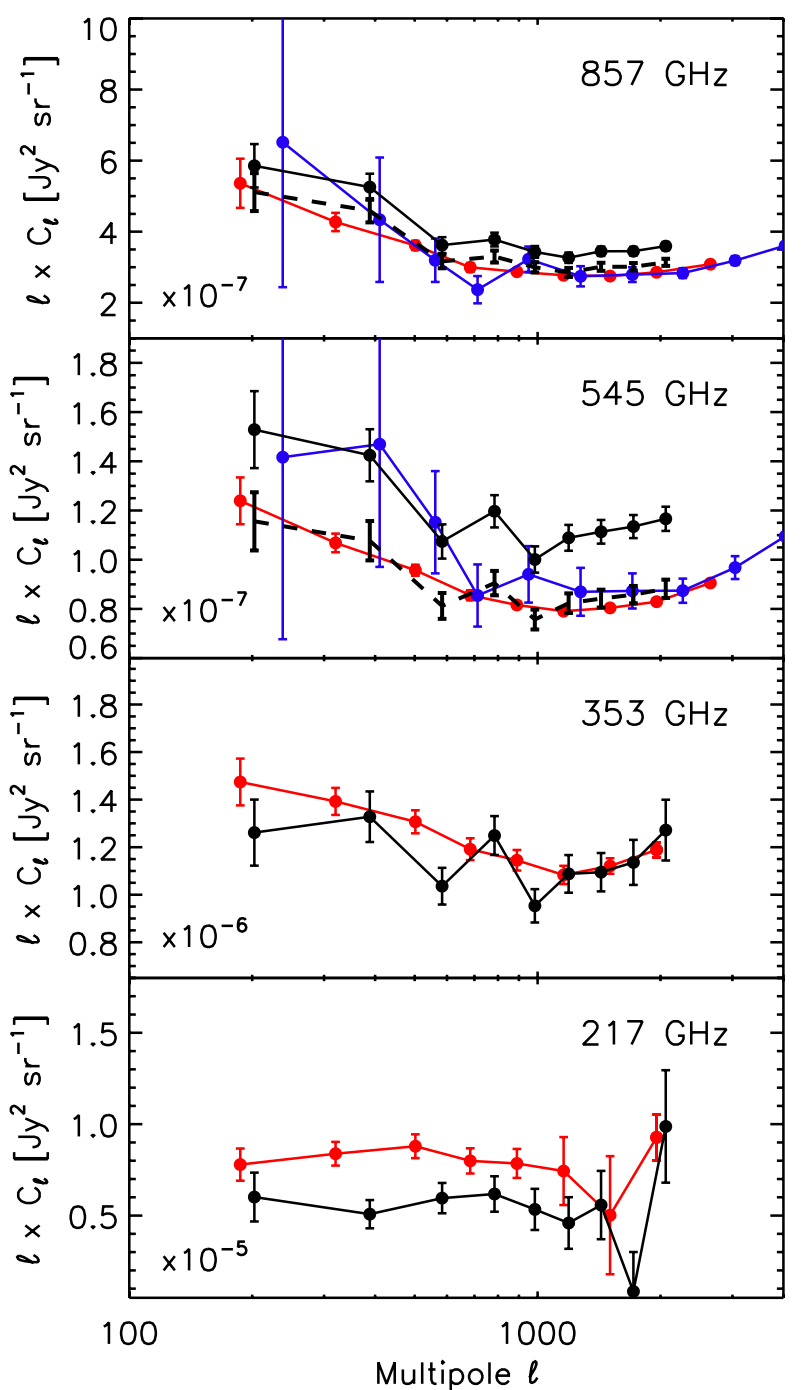

Fig. 17. Comparison of the CIB auto-power spectra measured using SPIRE (blue dots, Viero et al. 2013b), earlier Planck data (Planck Collaboration XVIII 2011, black dots) and in this paper (red circles). The SPIRE data have been colour corrected to be compared with HFI (see text). The dashed lines show the Planck Collaboration XVIII (2011) CIB measurements, rescaled at 857 and $545 \mathrm{GHz}$ by the photometric re-calibration factors $\left(1.07^{2}\right.$ and $1.15^{2}$, for the power spectra at 857 and $545 \mathrm{GHz}$, respectively, see Planck Collaboration VIII 2014). At $217 \mathrm{GHz}$, the difference between the black and red points is due to the tSZ and CIB contamination of the CMB template that is now corrected for. For display purpose, power spectra have been multiplied by the number given at the bottom-left side of each panel.

the recent average temperatures of star-forming galaxies found by Viero et al. (2013a) up to $z \sim 4$, the temperature of the effective SEDs is a bit low while the temperature of the SEDs of the extended halo model is a bit high. Knowing the SEDs of the galaxies that are responsible for the bulk of the CIB is the principal limitation in our modelling framework. Accurate future measurement of the SEDs will be crucial to properly estimate the obscured star formation rate density at high redshift from the CIB anisotropies. This is important if one wants to determine whether or not the bulk of the star formation is obscured at high redshift, and whether the UV and Lyman-break galaxy populations are a complete tracer of the star formation in the early Universe.

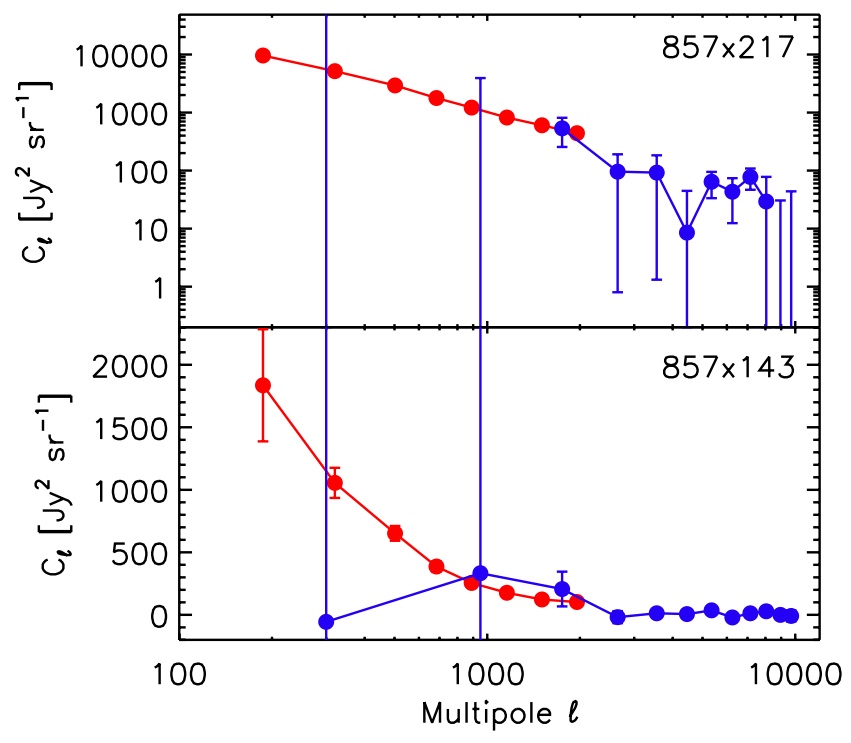

Fig. 18. Planck (red dots) and BLAST $\times$ ACT (blue dots from Hajian et al. 2012) CIB power spectra. Only the clustered CIB is shown (the shot-noise contributions have been removed, since they are very different in the two measurements). No colour corrections have been applied between HFI channels, and the $218 \mathrm{GHz}$ (ACT) and $857 \mathrm{GHz}$ (BLAST) channels. Note that the $y$-axis for the $857 \times 143$ cross-correlation is on a linear scale, since the BLAST $\times$ ACT measurement has negative values (due to the shot-noise removal).

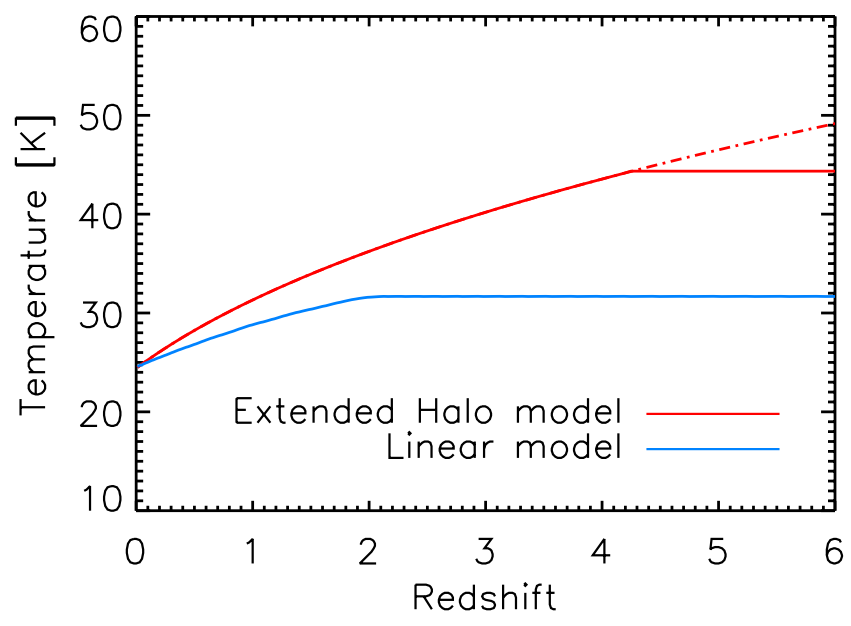

Fig. 19. Redshift evolution of the dust temperature of the effective SEDs used in the linear model (blue continuous line) and of the SEDs fit in the extended halo model (red continuous line with $z_{\text {break }}=4.2$, red dotdashed line without any redshift break).

\subsection{CIB non-Gaussianity}

Lacasa et al. (2012) proposed a phenomenological prescription for the CIB bispectrum based on its power spectrum, namely

$b_{\ell_{1} \ell_{2} \ell_{3}}=\alpha \sqrt{C_{\ell_{1}} C_{\ell_{2}} C_{\ell_{3}}}$,

where $\alpha$ is a dimensionless parameter quantifying the intrinsic level of non-Gaussianity. Using the best-fit power spectrum of the CIB model described in Sect. 5.5, we fitted this parameter $\alpha$ through a $\chi^{2}$ minimization using the covariance matrix described in Sect. 4.3. The resulting best-fit $\alpha$, its error bar (computed using a Fisher matrix analysis), and the $\chi^{2}$ value of the best fit can be found in Table 12 . 
Table 12. Best-fit amplitude parameter for the bispectrum prescription (Eq. (61)), and the values of $\chi^{2}$ and number of degrees of freedom associated with the fit.

\begin{tabular}{cccc}
\hline \hline Band & $\alpha$ & $\chi^{2}$ & $N_{\text {d.o.f. }}$ \\
\hline $217 \mathrm{GHz}$ & $(1.90 \pm 0.5) \times 10^{-3}$ & 21.4 & 37 \\
$353 \mathrm{GHz}$ & $(1.21 \pm 0.07) \times 10^{-3}$ & 45.5 & 39 \\
$545 \mathrm{GHz}$ & $(1.56 \pm 0.06) \times 10^{-3}$ & 95.9 & 35 \\
\hline
\end{tabular}

Table 13. Best-fit amplitude and index for a power-law fit to the bispectra, as well as the associated correlation, $\chi^{2}$ value of the fit and number of degrees of freedom.

\begin{tabular}{|c|c|c|c|c|c|}
\hline Band & $A\left[\mathrm{Jy}^{3} \mathrm{sr}^{-1}\right]$ & Index & Correlation & $x^{2}$ & $N_{\text {d.o.f }}$ \\
\hline $217 \mathrm{GHz}$ & $(1.46 \pm 0.68) \times 10^{1}$ & $0.822 \pm 0.145$ & $81.4 \%$ & 20.6 & 36 \\
\hline $353 \mathrm{GHz}$ & $(5.06 \pm 0.49) \times 10^{2}$ & $0.882 \pm 0.070$ & $82.3 \%$ & 34.3 & 38 \\
\hline $545 \mathrm{GHz}$ & $(1.26 \pm 0.09) \times 10^{4}$ & $0.814 \pm 0.050$ & $85.4 \%$ & 82.8 & 34 \\
\hline
\end{tabular}

The consistency of $\alpha$ across frequencies shows that the measured bispectrum has a frequency dependence consistent with that of the power spectrum. The best-fit $\alpha$ values are consistent with the values predicted using the number counts model of Béthermin et al. (2011). The best-fit $\alpha$ s are of the same order, although a little lower than those found by Lacasa et al. (2012) on simulations by Sehgal et al. (2010), since they found $\alpha \simeq$ $3 \times 10^{-3}$. This indicates a lower level of CIB non-Gaussianity than in the simulations by Sehgal et al. (2010).

The $\chi^{2}$ value of the fit shows that the prescription does not provide a very good model of the data as frequency increases; visual inspection reveals that this mainly comes from the fact that the measured bispectrum has a steeper slope than the prescription. To quantify the slope of the measured bispectrum, we fit a power law to the measurements, i.e.,

$b_{\ell_{1} \ell_{2} \ell_{3}}=A \times\left(\frac{\ell_{1} \ell_{2} \ell_{3}}{\ell_{0}^{3}}\right)^{-n}$,

where we chose as the pivot scale $\ell_{0}=320$, which is the centre of the second multipole bin. Table 13 presents the obtained bestfit values for the amplitude $A$ and the index $n$, as well as their error bars and correlation (computed again with Fisher matrices) and the $\chi^{2}$ value.

The power law provides a significantly better fit to the data than the prescription of Eq. (61), having lower best-fit $\chi^{2}$ values. The indices obtained are coherent between frequencies, and significantly steeper than the Eq. (61) prescription, which is $n \sim 0.6$ (since $C_{\ell} \propto \ell^{-1.2}$ ).

There is no sign of flattening of the bispectrum, showing that the shot-noise contribution is subdominant in this multipole range. This is consistent with shot-noise estimates based on the number counts model of Béthermin et al. (2011).

A detection of CIB non-Gaussianity has recently been reported by Crawford et al. (2014). In that paper, they used the prescription proposed by Lacasa et al. (2012) to give the amplitude of the bispectrum. In comparison with this analysis, we provide a higher detection significance, and at several frequencies. Most importantly, we find an indication that the CIB bispectrum is steeper than the prescription of Lacasa et al. (2012), although well fitted by a power law. However, our steeper CIB bispectrum at $217 \mathrm{GHz}$, extrapolated up to $\ell=2000$, is compatible with the Crawford et al. (2014) bispectrum measurement at $\ell=2000$ within $1 \sigma$. The steeper slope may be an indication that the contribution of more massive haloes to the CIB bispectrum is smaller than in the models studied by Lacasa et al. (2014) and Pénin et al. (2014).

\section{Conclusions}

We have presented new measurements of the CIB anisotropies with Planck. Owing to the exceptional quality of the data, and using a complete analysis of the different steps that lead to the CIB anisotropy power spectra, we have been able to measure the clustering of dusty, star-forming galaxies at 143, 217, 353, 545, and $857 \mathrm{GHz}$, with unprecedented precision. For the fist time we also measured the bispectrum from $\ell \simeq 130$ to 900 at 217, 353, and $545 \mathrm{GHz}$. The CIB power spectrum is also measured with IRAS at $3000 \mathrm{GHz}$.

We worked on 11 independent fields, chosen to have high angular-resolution Hi data and low foreground contamination. The total areas used to compute the angular power spectrum is about $2240 \mathrm{deg}^{2}$. This improves over previous Planck and Herschel analyses by more than an order of magnitude. For the bispectrum, the total area is about $4400 \mathrm{deg}^{2}$.

To obtain the CIB, the HFI and IRAS maps were cleaned using two templates: HI for Galactic cirrus; and the Planck $100 \mathrm{GHz}$ map for CMB. We used new HI data that covers very large portions of the sky. The large areas forced us to build a dust model that takes into account the submillimetre-HI emissivity variations. However, because the HI is not a perfect tracer of dust emission (e.g., the dark gas), and clearly contains dustdeficient clouds, we had to reduce the sky fraction to the lowest HI column-density parts of the sky. The $100 \mathrm{GHz}$ Planck channel, cleaned of Galactic dust and sources, and then filtered, provides a good template for the CMB. This is because it has an angular resolution close to the higher frequency channels, from which we measure the CIB, and has the advantage of being an "internal" template, meaning that its noise, data reduction processing steps, photometric calibration, and beam are all well known. It has the drawback of contaminating CIB measurements with tSZ signal and spurious CIB coming from the correlation between the CIB at $100 \mathrm{GHz}$, and the CIB at higher frequency. The tSZ and spurious CIB corrections are relatively small for frequencies $v \geq 217 \mathrm{GHz}$. At $143 \mathrm{GHz}$, while the tSZ and tSZ-CIB corrections are still rather small (lower than $20 \%$ ), the spurious CIB is a very large correction (between 30 and $70 \%$ at intermediate scales) due to the high level of CIB correlation between 100 and $143 \mathrm{GHz}$. Thus, the $143 \mathrm{GHz}$ CIB measurements strongly rely on the CIB model used to compute the correction.

Due to dust contamination at high frequency, as well as radio sources, SZ and CIB at low frequency, we conservatively restrict our bispectrum measurement to the three frequencies 217, 353, and $545 \mathrm{GHz}$. We measure the bispectrum due to the clustering of dusty star forming galaxies from $\ell \simeq 130$ to 900 . It is detected with a very high significance, $>28 \sigma$ for all configurations, and even $>4.5 \sigma$ for individual configurations at $545 \mathrm{GHz}$. Such measurements are completely new; they open a window for constraining models of CIB source emission that we have not yet fully explored in this paper.

We developed two approaches for modelling the CIB anisotropies. The first takes advantage of the accurate measurement of CIB anisotropies performed with Planck and IRIS at large angular scales, and uses only the linear part of the power spectra. The second approach uses the measurements at all angular scales, and takes advantage of the frequency coverage, to constrain a halo model with a luminosity-mass dependence. We 
find that both models give a very good fit to the data. Our main findings are as follows.

- The models give strong constraints on the star formation history up to redshift $\sim 2.5$. At higher redshift, the accuracy of the star formation history measurement is strongly degraded by the uncertainty on the SED of CIB galaxies. An accurate measurement of SEDs of galaxies that are responsible for the bulk of the CIB will be crucial to estimate properly the obscured star-formation rate density at high redshifts from the CIB anisotropies.

- As found in other recent studies, haloes of mass $M_{\mathrm{eff}}=4 \times$ $10^{12} M_{\odot}$ appear to be the most efficient at actively forming stars.

- CIB galaxies have warmer temperatures as redshift increases $\left(T_{\mathrm{d}}(z)=24.4 \times(1+z)^{0.36} \mathrm{~K}\right.$ in the extended halo model $)$. This is compatible with the most recent Herschel observations, and can be explained by a harder interstellar radiation field in high- $z$ galaxies.

- The same halo occupation distribution can simultaneously fit all power spectra. However, the 1-halo term is significantly reduced compared to previous studies (Pénin et al. 2012a; Planck Collaboration XVIII 2011). This is due to a lower contribution to the clustering from low $-z$ massive haloes, as also observed in Béthermin et al. (2013).

We find that the CIB bispectrum is steeper than the prescription developed by Lacasa et al. (2012). Just like the reduction of the 1-halo term in the power spectrum, this may be an indication that the contribution of massive haloes to the CIB bispectrum is smaller than in the models studied by Lacasa et al. (2014) and Pénin et al. (2014). The bispectrum is quite well fitted by a power law. This can be used to provide valuable constraints on the potential contamination of measurements of the primordial CMB bispectrum on large scales.

While our component separation process is successful in extracting the CIB from the maps, the next step is to use a full multi-frequency fitting procedure to separate the CIB power spectrum and bispectrum, from the tSZ (and kSZ) effects, the $\mathrm{CMB}$, and the extragalactic source contribution. Simultaneously taking into account all the components will improve our ability to separate them. The goal is to give unprecedented limits on the reionization history of the Universe, as well as understanding the history of star formation in dark matter haloes.

Acknowledgements. The development of Planck has been supported by: ESA; CNES and CNRS/INSU-IN2P3-INP-PNCG (France); ASI, CNR, and INAF (Italy); NASA and DoE (USA); STFC and UKSA (UK); CSIC, MICINN and JA (Spain); Tekes, AoF and CSC (Finland); DLR and MPG (Germany); CSA (Canada); DTU Space (Denmark); SER/SSO (Switzerland); RCN (Norway); SFI (Ireland); FCT/MCTES (Portugal); and PRACE (EU). A description of the Planck Collaboration and a list of its members with the technical or scientific activities they have been involved into, can be found at http://www.rssd. esa. int/index . php? project=PLANCK\&page=PlanckCollaboration. The Parkes radio telescope is part of the Australia Telescope National Facility which is funded by the Commonwealth of Australia for operation as a National Facility managed by CSIRO. Some HI data used in this paper are based on observations with the $100 \mathrm{~m}$ telescope of the MPIfR (Max-Planck-Institut für Radioastronomie) at Effelsberg.

\section{Appendix A: H I data}

We describe in this Appendix the three HI surveys that we use to remove Galactic dust contamination from the frequency maps. The 21-cm HI spectra were obtained with: (1) the 100-m Green Bank Telescope (GBT); (2) the Parkes 64-m telescope; and (3) the Effelsberg 100-m radio telescope.

\section{A.1. GBT observations and data preparation}

The GBT HI maps are created from HI spectral observations with the Green Bank Telescope, the details of which can be found in Boothroyd et al. (2011) and Martin et al. (in prep.). Over $800 \mathrm{deg}^{2}$ were mapped between 2002 and 2010, each following roughly the same observing strategy. The high Galactic latitude fields were mapped using scans along lines of constant declination or constant Galactic latitude at a scan rate equalling $3.5^{\prime}$ every $4 \mathrm{~s}$. Each subsequent scan is offset by $3.5^{\prime}$ in the corresponding orthogonal direction. This strategy results in a rectangular region of the HI sky sampled every $3.5^{\prime}$. Some of the regions have been scanned in this way multiple times, in order to increase the $\mathrm{S} / \mathrm{N}$, and to investigate the stability of the system (Boothroyd et al. 2011).

The spectra are first converted from their antenna temperature scale to a brightness temperature $\left(T_{\mathrm{b}}\right)$ scale. This involves calibration and stray radiation corrections, as discussed in Boothroyd et al. (2011). All $T_{\mathrm{b}}$ spectra for a corresponding region are assigned to a $3.5^{\prime}$ Sanson-Flamsteed-projection grid (SFL-projection) using convolution with an optimized tapered Bessel function, in order to minimize noise on spatial scales smaller than the beam (Mangum et al. 2007). Note that this observing strategy and gridding choice results in a final cube resolution of $9.55^{\prime} \times 9.24^{\prime}$, slightly broader than the inherent $9.1^{\prime} \times 9.0^{\prime}$ GBT $21-\mathrm{cm}$ beam.

Each spectrum is recorded using in-band frequencyswitching, resulting in velocity coverage of $-450 \mathrm{~km} \mathrm{~s}^{-1} \leq$ $V_{\mathrm{LSR}} \leq 355 \mathrm{~km} \mathrm{~s}^{-1}$, with very flat baselines. Any residual baseline is removed on a pixel-by-pixel basis by fitting the emissionfree channels in the final calibrated cube using a third-order polynomial.

The archival LH2 field was observed using a $3^{\prime}$ grid pattern with the GBT spectral processor and was calibrated accordingly. As the residual baseline behaviour is different for the archival data, only a linear polynomial was fit to the emission-free channels. The rest of the processing was identical to that described above.

The individual $0.8 \mathrm{~km} \mathrm{~s}^{-1}$ spectral channels of the cubes are integrated to convert the $T_{\mathrm{b}}$ spectra into $N_{\mathrm{HI}}$ maps:

$N_{\mathrm{HI}}(x, y)=1.823 \times 10^{18} \sum_{v} T_{\mathrm{b}}(x, y, v) \tau\left(T_{\mathrm{s}}\right) \delta v$,

where the sum is over a given velocity range, $v$, and $\delta v$ is the $0.80 \mathrm{~km} \mathrm{~s}^{-1}$ channel spacing. The quantity $\tau$ is the opacity correction for spin temperature, $T_{\mathrm{s}}$ :

$\tau\left(T_{\mathrm{s}}\right)=-\ln \left(1-T_{\mathrm{b}} / T_{\mathrm{s}}\right)$.

For the adopted value of $T_{\mathrm{s}}=80 \mathrm{~K}$, these corrections are all less than 5\% for our CIB fields (Planck Collaboration XVIII 2011).

The velocity ranges over which the integrations are performed are selected using the observed velocity structure in each of the cubes. The models presented here subdivide each cube into three velocity-selected components: a local component; intermediate-velocity clouds, IVCs; and high-velocity clouds, HVCs. Divisions between components are distinguishable by reductions in structure (as measured through the standard deviation of individual channel maps) as one progresses through the data cube, channel by channel. More details can be found in Planck Collaboration XVIII (2011).

\section{A.2. GASS observations and data preparation}

The GASS survey is a $21-\mathrm{cm}$ line survey covering the southern sky for all declinations $\delta \lesssim 1^{\circ}$. The observations were made with 
the multibeam system on the 64-m Parkes radio telescope. The intrinsic angular resolution of the data is $14.4^{\prime}$ full width at half maximum (FWHM). The velocity resolution is $1.0 \mathrm{~km} \mathrm{~s}^{-1}$ and the useful bandpass covers a velocity range $\left|v_{\mathrm{lsr}}\right| \lesssim 468 \mathrm{~km} \mathrm{~s}^{-1}$ for all of the observations; some data cover up to $\left|v_{\mathrm{lsr}}\right| \lesssim 500 \mathrm{~km} \mathrm{~s}^{-1}$. GASS is the most sensitive, highest angular resolution largescale survey of Galactic HI emission ever made in the southern sky. The observations are described in McClure-Griffiths et al. (2009). We used data from the final data release (Kalberla et al. 2010) that were corrected for instrumental effects and radio-frequency interference (RFI). The data were gridded on a Cartesian grid on the Magellanic stream (MS) coordinate system as defined by Nidever et al. (2008). To minimize the noise and eliminate residual instrumental problems, we calculated a second $3 \mathrm{D}$ data cube with a beam of $0.5^{\circ} \mathrm{FWHM}$, smoothing at the same time in velocity by $8 \mathrm{~km} \mathrm{~s}^{-1}$. Emission below a $5 \sigma$ level of $30 \mathrm{mK}$ in the smoothed data-cube was considered as insignificant and was accordingly zeroed. For details in data processing and analysis see Venzmer et al. (2012).

When looking at the HI data cube in the southern sky, one of the most prominent structures is linked to hydrogen gas in the Magellanic stream, in the disks of the Magellanic clouds, and in the stream's leading arm. In particular, the Magellanic stream, stretches over $100^{\circ}$ behind the Large and Small Magellanic clouds. We thus need to remove this contamination to be able to use the HI as a tracer of Galactic dust.

Aiming to separate Galactic emission from the observations of the Magellanic system we calculated the expected Milky Way emission according to the model of Kalberla \& Dedes (2008). Velocities for components in direction towards the southern Galactic pole were shifted by $-5 \mathrm{~km} \mathrm{~s}^{-1}$ to mimic the apparent infall (see Weaver 1974). Comparisons between the emission and the model at two particular velocities in the Galactic standard of rest frame are shown in Fig. A.1. The strong Galactic emission can be traced to weak extended line wings that are well represented by the model. It is therefore feasible to use the model to predict regions in the 3D data cube that are most probably occupied by Milky Way emission. We used a clip level of $60 \mathrm{mK}$, the lowest isophote in Fig. A.1 that delineates the disk emission. Such a treatment extracts most of the Galactic emission, however we must take into account the fact that at positions with strongly blended lines Magellanic emission also gets included. A higher clip level would minimize this problem, although, at the same time the wings of the Galactic emission would be affected. The chosen clip level of $60 \mathrm{mK}$ (comparable to the instrumental noise), is a good compromise.

As the final step in the reduction of the GASS data we integrated the HI emission over the appropriate velocity range for each individual position in the $N_{\text {side }}=512$ HEALPix database to obtain column densities of the Galactic gas. To avoid any interpolation errors, we extracted profiles from the original GASS database; all intermediate data products that have been described above served only to discriminate Galactic emission from the Magellanic stream. Due to the finite beam size of the Parkes telescope and the Gaussian weighting that was used for the gridding, the effective resolution of the HEALPix data is $16.2^{\prime}$ FWHM. Present a detailed analysis of the dust-HI correlation in this field.

\section{A.3. EBHIS observations and data analyses}

The Effelsberg-Bonn Hi Survey (EBHIS) comprises an HI survey of the entire northern sky for all declinations $\delta \gtrsim-5^{\circ}$ with the Effelsberg 100-m telescope. The bandwidth of $100 \mathrm{MHz}$
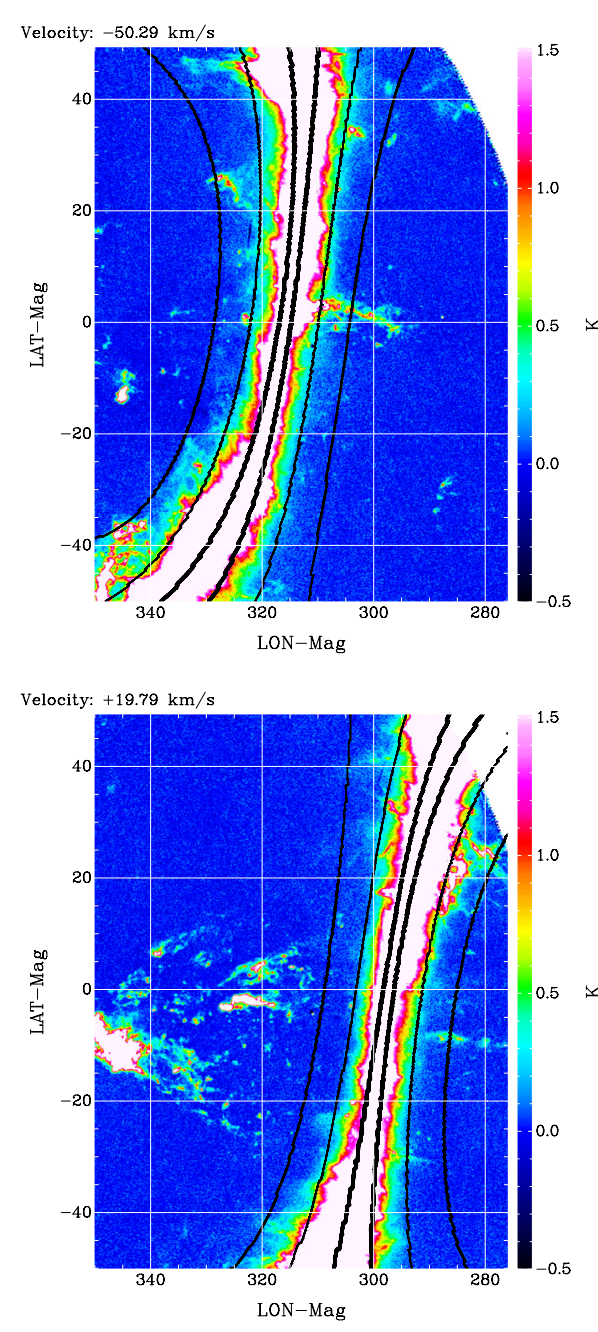

Fig. A.1. Comparison of observed HI emission with the Galactic model (black isophotes are for the expected emission at levels of 0.06, 0.6 and $6.0 \mathrm{~K}$ ). Magellanic coordinates (longitude and latitude) are used. Two examples for channel maps at Galactic standard of rest velocities of 19.8 and $-50.3 \mathrm{~km} \mathrm{~s}^{-1}$ are given.

covers $-1000 \mathrm{~km} \mathrm{~s}^{-1} \leq v_{\mathrm{LSR}} \leq 19000 \mathrm{~km} \mathrm{~s}^{-1}$. This allows us to study the detailed Milky Way Hi structure as well as the local Universe up to a redshift of $z \simeq 0.07$ (Kerp et al. 2011), with an effective velocity resolution of about $2.1 \mathrm{~km} \mathrm{~s}^{-1}$.

We selected from the early survey data a clean high Galactic latitude field. The observations of the field of interest were performed during the summer of 2011. Following the standard observing strategy (Kerp et al. 2011) individual fields of $25 \mathrm{deg}^{2}$ were measured. In addition to the data reduction and calibration pipeline of Winkel et al. (2010), the HI data were corrected with an improved RFI mitigation detection algorithm and the absolute offsets between the individual fields were minimized to a level of $N_{\mathrm{HI}} \leq 3 \times 10^{18} \mathrm{~cm}^{-2}$. As for the GBT fields, the EBHIS data were put on a $3.5^{\prime}$ SFL-projection grid.

The overall HI emission in this field can be characterized by intermediate- and low-velocity emission populating the radial velocity range $-80 \mathrm{~km} \mathrm{~s}^{-1} \leq v_{\mathrm{LSR}} \leq+20 \mathrm{~km} \mathrm{~s}^{-1}$.

The area of interest is about $130 \mathrm{deg}^{2}$ with HI column densities below $N_{\mathrm{HI}}=2 \times 10^{20} \mathrm{~cm}^{-2}$. The total HI column density range is $0.98 \times 10^{20} \mathrm{~cm}^{-2} \leq N_{\mathrm{HI}} \leq 7.5 \times 10^{20} \mathrm{~cm}^{-2}$. Accordingly we expect infrared excess emission associated with molecular hydrogen towards areas of $N_{\mathrm{HI}} \geq 3 \times 10^{20} \mathrm{~cm}^{-2}$, while the 
low-column density regions should allow us to study the emission associated with the CIB.

In the first step we performed a linear fit of the EBHIS HI data integrated across the velocity range $-160 \mathrm{~km} \mathrm{~s}^{-1} \leq v_{\mathrm{LSR}} \leq$ $+160 \mathrm{~km} \mathrm{~s}^{-1}$ to the HFI $857 \mathrm{GHz}$ map: $I_{857}=a \times N_{\mathrm{HI}}+b$, for $N_{\mathrm{HI}} \leq 2 \times 10^{20} \mathrm{~cm}^{-2}$. The residual map shows dust excess, as expected when the total infrared emissivity traces $\mathrm{HI}$ and $\mathrm{H}_{2}$ (Planck Collaboration XIX 2011; Planck Collaboration XXIV 2011). In agreement with our expectation dust excess clouds show up with HI column densities of $N_{\mathrm{HI}} \geq 3 \times 10^{20} \mathrm{~cm}^{-2}$. But surprisingly, the residual map shows dust-deficient HI clouds that populate the column density range $2.0 \leq N_{\mathrm{HI}} \leq 5.5 \times$ $10^{20} \mathrm{~cm}^{-2}$. The HI emission of these dust-deficient clouds can be characterized by brightness temperatures $T_{\mathrm{B}}>15 \mathrm{~K}$ and $\Delta v(F W H M) \leq 5 \mathrm{~km} \mathrm{~s}^{-1}$. These HI lines are associated with individual, cold IVCs. A positional correlation between the dustdeficient regions and the IVCs show a very strong correlation. We evaluated the dust deficiency of the IVCs and found that they are not "under-luminous", but infrared dark.

To extract the CIB in this field we produced a mask excluding the excess as well as the dust-deficient clouds. About $90 \mathrm{deg}^{2}$ are suitable for CIB analysis.

\section{Appendix B: From infrared luminosity density to emissivities}

The emissivity, often written $j_{v}$, is given by (Pénin et al. 2012a)

$j_{v}(z)=\frac{1+z}{\mathrm{~d} \chi / \mathrm{d} z} \int S_{v} \frac{\mathrm{d}^{2} N}{\mathrm{~d} S_{v} \mathrm{~d} z} \mathrm{~d} S_{v}$,

where $\chi$ is the comoving distance, $S_{v}$ the flux density, and $\mathrm{d}^{2} N / \mathrm{d} S_{v} \mathrm{~d} z$ the number of sources per flux density and redshift interval. If there are several types of galaxy (labelled by " $t$ ") with different SEDs ${ }^{9}$, one can rewrite Eq. (B.1) as

$j_{v}(z)=\frac{1+z}{\mathrm{~d} \chi / \mathrm{d} z} \sum_{t} \int S_{v} \frac{\mathrm{d}^{2} N_{t}}{\mathrm{~d} S_{v} \mathrm{~d} z} \mathrm{~d} S_{v}$.

We then introduce $s_{v, t}$ the flux density of an $L_{\mathrm{IR}}=1 L_{\odot}$ source with an SED of a given type. This quantity varies only with $z$ for a given type of SED. We can thus easily change the variable $S_{v}$ to $L_{\mathrm{IR}}$ in the integral:

$j_{v}(z)=\frac{1+z}{\mathrm{~d} \chi / \mathrm{d} z} \sum_{t} s_{v} \int L_{\mathrm{IR}} \frac{\mathrm{d}^{2} N_{t}}{\mathrm{~d} L_{\mathrm{IR}} \mathrm{d} z} \mathrm{~d} L_{\mathrm{IR}}$.

We can slightly modify this expression so that it contains the bolometric infrared luminosity function of each galaxy type $\mathrm{d}^{2} N_{t} / \mathrm{d} L_{\mathrm{IR}} \mathrm{d} V$ :

$j_{v}(z)=\frac{1+z}{\mathrm{~d} \chi / \mathrm{d} z} \frac{\mathrm{d} V}{\mathrm{~d} z} \sum_{\mathrm{t}} s_{v} \int L_{\mathrm{IR}} \frac{\mathrm{d}^{2} N_{t}}{\mathrm{~d} L_{\mathrm{IR}} \mathrm{d} V} \mathrm{~d} L_{\mathrm{IR}}$,

where $V$ is the comoving volume. We can simplify this expression assuming a flat $\Lambda \mathrm{CDM}$ Universe with

$\frac{\mathrm{d} \chi}{\mathrm{d} z}=\frac{c}{H_{0} \sqrt{\Omega_{\mathrm{m}}(1+z)^{3}+\Omega_{\Lambda}}}$

and

$\frac{\mathrm{d} V}{\mathrm{~d} z}=\frac{c \chi^{2}}{H_{0} \sqrt{\Omega_{\mathrm{m}}(1+z)^{3}+\Omega_{\Lambda}}}=\frac{\mathrm{d} \chi}{\mathrm{d} z} \chi^{2}$.

\footnotetext{
9 The result is also true if there is a scatter in temperature. Each range of temperature is then considered as a type.
}

We can thus simplify Eq. (B.4):

$j_{v}(z)=(1+z) \chi^{2} \sum_{t} s_{v} \int L_{\mathrm{IR}} \frac{\mathrm{d}^{2} N_{t}}{\mathrm{~d} L_{\mathrm{IR}} \mathrm{d} V} \mathrm{~d} L_{\mathrm{IR}}$.

We introduce the contribution to the infrared luminosity density of a given type of SED:

$\rho_{\mathrm{IR}, t}=\frac{\mathrm{d}^{2} N_{t}}{\mathrm{~d} L_{\mathrm{IR}} \mathrm{d} V} \mathrm{~d} L_{\mathrm{IR}}$

where $\rho_{\mathrm{IR}}$ is the total infrared luminosity density. We can assume a simple conversion between $L_{\mathrm{IR}}$ and $\rho_{\mathrm{SFR}}$, the star formation rate density, using the Kennicutt (1998) constant $K$. Then Eq. (B.7) can be rewritten as:

$j_{v}(z)=(1+z) \chi^{2} \frac{\rho_{\mathrm{SFR}}}{K} \times\left(\frac{\sum_{t} s_{v} \rho_{\mathrm{IR}, t}}{\sum_{t} \rho_{\mathrm{IR}, t}}\right)$,

where $\sum_{t} s_{v} \rho_{\mathrm{IR}, t} / \sum_{t} \rho_{\mathrm{IR}, t}$ is the effective SED of infrared galaxies (noted $s_{v, \text { eff }}$ ), i.e., the mean SED using a weighting by the contribution to the infrared luminosity density. Here we use the model of Béthermin et al. (2012a) to compute the contribution at each redshift. This model is based on the observed strong correlation between stellar mass and star formation rate in these galaxies (called main-sequence galaxies), and new SED templates from Magdis et al. (2012). We finally obtain:

$j_{v}(z)=\rho_{\mathrm{SFR}}(z) \frac{(1+z) \chi^{2}(z) s_{v, \mathrm{eff}}(z)}{K}$.

\section{Appendix C: Effective bias notion for the linear model}

We discuss here the link between the approach chosen to model the 2-halo term in Sects. 5.4 (linear model) and 5.5 (HOD model). We consider for simplicity only the case of the auto-spectrum, but this can be easily generalized to the crossspectrum. Equation (48) of the HOD model can be re-written as

$P_{2 \mathrm{~h}}^{v v}(k, z)=\int\left(\frac{a}{\chi}\right)^{2}\left(\frac{\mathrm{d} j}{\mathrm{~d} M}(v, z) b(M, z)\right)^{2} P_{\operatorname{lin}}(k, z) \mathrm{d} \chi$,

where $\mathrm{d} j / \mathrm{d} M$ is the differential contribution of haloes of mass $M$, at redshift $z$, to the emissivity. It can be computed using

$\frac{\mathrm{d} j}{\mathrm{~d} M}(v, z)=\frac{\mathrm{d} N}{\mathrm{~d} M} \times\left\{f_{v}^{\mathrm{cen}}(M, z)+f_{v}^{\mathrm{sat}}(M, z)\right\}$.

The total emissivity is thus

$j(v, z)=\int \frac{\mathrm{d} j}{\mathrm{~d} M}(v, z) \mathrm{d} M$.

In order to simplify Eq. (C.1), we introduce an effective bias,

$b_{\text {eff }}(z)=\frac{\int \frac{\mathrm{d} j}{\mathrm{~d} M}(v, z) b(M, z) \mathrm{d} M}{\int \frac{\mathrm{d} j}{\mathrm{~d} M}(v, z) \mathrm{d} M}$.

This effective bias is the mean bias of haloes hosting the infrared galaxies, weighted by their differential contribution to the emissivities. We finally obtain a simpler equation, which is used in the linear model:

$P_{2 \mathrm{~h}}^{v v}(k, z)=\int\left(\frac{a}{\chi}\right)^{2} j^{2}(v, z) b_{\mathrm{eff}}^{2} P_{\text {lin }}(k, z) \mathrm{d} \chi$. 


\section{Appendix D: Tables}

Table D.1. Cross-power spectra and error bars for all pairs of frequencies, measured on Galactic dust- and CMB-free residual maps.

\begin{tabular}{|c|c|c|c|c|c|c|c|}
\hline & \multirow[b]{2}{*}{$\ell_{\text {cent }}$} & \multicolumn{6}{|c|}{ Cross-power spectra, $C_{\ell}$, and uncertainty $\left[\mathrm{Jy}^{2} \mathrm{sr}^{-1}, \nu \mathrm{I}_{v}=\mathrm{cst}\right]$} \\
\hline & & $3000 \mathrm{GHz}$ & $857 \mathrm{GHz}$ & $545 \mathrm{GHz}$ & $353 \mathrm{GHz}$ & $217 \mathrm{GHz}$ & $143 \mathrm{GHz}$ \\
\hline \multirow[t]{9}{*}{$3000 \mathrm{GHz}$} & 187 & $(4.35 \pm 1.34) \times 10^{5}$ & $(1.12 \pm 0.85) \times 10^{5}$ & $(3.94 \pm 3.88) \times 10^{4}$ & $(8.92 \pm 12.4) \times 10^{3}$ & $(5.59 \pm 26.7) \times 10^{2}$ & r. \\
\hline & 320 & $(7.89 \pm 3.14) \times 10^{4}$ & $(7.49 \pm 2.09) \times 10^{4}$ & $(3.04 \pm 0.85) \times 10^{4}$ & $(1.13 \pm 0.27) \times 10^{4}$ & $(2.34 \pm 0.71) \times 10^{3}$ & \\
\hline & 502 & $(3.46 \pm 1.15) \times 10^{4}$ & $(3.10 \pm 0.79) \times 10^{4}$ & $(1.23 \pm 0.33) \times 10^{4}$ & $(4.49 \pm 1.15) \times 10^{3}$ & $(1.18 \pm 0.34) \times 10^{3}$ & $\ldots$ \\
\hline & 684 & $(2.90 \pm 0.70) \times 10^{4}$ & $(2.24 \pm 0.47) \times 10^{4}$ & $(9.35 \pm 2.10) \times 10^{3}$ & $(2.87 \pm 0.73) \times 10^{3}$ & $(6.88 \pm 2.61) \times 10^{2}$ & $\ldots$ \\
\hline & 890 & $(1.87 \pm 0.47) \times 10^{4}$ & $(1.62 \pm 0.30) \times 10^{4}$ & $(6.61 \pm 1.34) \times 10^{3}$ & $(1.99 \pm 0.45) \times 10^{3}$ & $(2.34 \pm 1.66) \times 10^{2}$ & \\
\hline & 1158 & $(1.43 \pm 0.40) \times 10^{4}$ & $(1.17 \pm 0.22) \times 10^{4}$ & $(4.71 \pm 0.93) \times 10^{3}$ & $(1.25 \pm 0.31) \times 10^{3}$ & $(3.55 \pm 1.43) \times 10^{2}$ & $\ldots$ \\
\hline & 1505 & $(1.09 \pm 0.41) \times 10^{4}$ & $(8.30 \pm 1.85) \times 10^{3}$ & $(3.12 \pm 0.76) \times 10^{3}$ & $(1.14 \pm 0.30) \times 10^{3}$ & $(2.41 \pm 1.07) \times 10^{2}$ & $\ldots$ \\
\hline & 1956 & $(1.05 \pm 0.58) \times 10^{4}$ & $(8.04 \pm 2.39) \times 10^{3}$ & $(3.16 \pm 1.00) \times 10^{3}$ & $(9.67 \pm 3.44) \times 10^{2}$ & $(2.58 \pm 1.14) \times 10^{2}$ & $\ldots$ \\
\hline & 2649 & $(1.06 \pm 1.04) \times 10^{4}$ & $(6.47 \pm 3.27) \times 10^{3}$ & $(2.61 \pm 1.38) \times 10^{3}$ & $\ldots$ & $\ldots$ & $\ldots$ \\
\hline \multirow[t]{11}{*}{$857 \mathrm{GHz}$} & 53 & $\ldots$ & $(1.49 \pm 1.27) \times 10^{6}$ & $(5.59 \pm 4.14) \times 10^{5}$ & $(1.70 \pm 1.17) \times 10^{5}$ & $(3.61 \pm 2.53) \times 10^{4}$ & $(5.15 \pm 11.6) \times 10^{3}$ \\
\hline & 114 & $\ldots$ & $(6.37 \pm 1.62) \times 10^{5}$ & $(2.67 \pm 0.57) \times 10^{5}$ & $(8.63 \pm 1.68) \times 10^{4}$ & $(1.91 \pm 0.38) \times 10^{4}$ & $(2.53 \pm 1.56) \times 10^{3}$ \\
\hline & 187 & $\ldots$ & $(2.87 \pm 0.37) \times 10^{5}$ & $(1.30 \pm 0.13) \times 10^{5}$ & $(4.18 \pm 0.39) \times 10^{4}$ & $(8.61 \pm 0.89) \times 10^{3}$ & $(1.16 \pm 0.45) \times 10^{3}$ \\
\hline & 320 & $\ldots$ & $(1.34 \pm 0.08) \times 10^{5}$ & $(6.36 \pm 0.30) \times 10^{4}$ & $(2.12 \pm 0.09) \times 10^{4}$ & $(4.61 \pm 0.22) \times 10^{3}$ & $(6.43 \pm 1.20) \times 10^{2}$ \\
\hline & 502 & $\ldots$ & $(7.20 \pm 0.26) \times 10^{4}$ & $(3.53 \pm 0.10) \times 10^{4}$ & $(1.20 \pm 0.03) \times 10^{4}$ & $(2.65 \pm 0.09) \times 10^{3}$ & $(4.26 \pm 0.59) \times 10^{2}$ \\
\hline & 684 & $\ldots$ & $(4.38 \pm 0.18) \times 10^{4}$ & $(2.21 \pm 0.07) \times 10^{4}$ & $(7.68 \pm 0.22) \times 10^{3}$ & $(1.62 \pm 0.06) \times 10^{3}$ & $(2.39 \pm 0.41) \times 10^{2}$ \\
\hline & 890 & $\ldots$ & $(3.23 \pm 0.09) \times 10^{4}$ & $(1.63 \pm 0.04) \times 10^{4}$ & $(5.66 \pm 0.12) \times 10^{3}$ & $(1.14 \pm 0.04) \times 10^{3}$ & $(1.55 \pm 0.30) \times 10^{2}$ \\
\hline & 1158 & $\ldots$ & $(2.40 \pm 0.05) \times 10^{4}$ & $(1.22 \pm 0.02) \times 10^{4}$ & $(4.12 \pm 0.07) \times 10^{3}$ & $(8.29 \pm 0.25) \times 10^{2}$ & $(1.29 \pm 0.23) \times 10^{2}$ \\
\hline & 1505 & $\ldots$ & $(1.83 \pm 0.03) \times 10^{4}$ & $(9.31 \pm 0.11) \times 10^{3}$ & $(3.19 \pm 0.04) \times 10^{3}$ & $(6.82 \pm 0.16) \times 10^{2}$ & $(1.13 \pm 0.16) \times 10^{2}$ \\
\hline & 1956 & $\ldots$ & $(1.46 \pm 0.02) \times 10^{4}$ & $(7.38 \pm 0.07) \times 10^{3}$ & $(2.50 \pm 0.03) \times 10^{3}$ & $(5.36 \pm 0.11) \times 10^{2}$ & $(1.03 \pm 0.15) \times 10^{2}$ \\
\hline & 2649 & $\ldots$ & $(1.16 \pm 0.01) \times 10^{4}$ & $(5.91 \pm 0.06) \times 10^{3}$ & $\ldots$ & $\ldots$ & $\ldots$ \\
\hline \multirow[t]{11}{*}{$545 \mathrm{GHz}$} & 53 & $\cdots$ & $\ldots$ & $(2.36 \pm 1.37) \times 10^{5}$ & $(7.46 \pm 3.90) \times 10^{4}$ & $(1.61 \pm 0.85) \times 10^{4}$ & $(2.46 \pm 3.80) \times 10^{3}$ \\
\hline & 114 & $\ldots$ & $\ldots$ & $(1.24 \pm 0.21) \times 10^{5}$ & $(4.12 \pm 0.63) \times 10^{4}$ & $(9.32 \pm 1.44) \times 10^{3}$ & $(1.35 \pm 0.57) \times 10^{3}$ \\
\hline & 187 & $\ldots$ & $\ldots$ & $(6.63 \pm 0.51) \times 10^{4}$ & $(2.16 \pm 0.15) \times 10^{4}$ & $(4.48 \pm 0.35) \times 10^{3}$ & $(6.11 \pm 1.78) \times 10^{2}$ \\
\hline & 320 & $\ldots$ & $\ldots$ & $(3.34 \pm 0.12) \times 10^{4}$ & $(1.15 \pm 0.04) \times 10^{4}$ & $(2.49 \pm 0.09) \times 10^{3}$ & $(3.51 \pm 0.52) \times 10^{2}$ \\
\hline & 502 & $\ldots$ & $\ldots$ & $(1.91 \pm 0.04) \times 10^{4}$ & $(6.73 \pm 0.15) \times 10^{3}$ & $(1.48 \pm 0.04) \times 10^{3}$ & $(2.38 \pm 0.28) \times 10^{2}$ \\
\hline & 684 & $\ldots$ & $\ldots$ & $(1.25 \pm 0.03) \times 10^{4}$ & $(4.48 \pm 0.09) \times 10^{3}$ & $(9.39 \pm 0.29) \times 10^{2}$ & $(1.37 \pm 0.21) \times 10^{2}$ \\
\hline & 890 & $\ldots$ & $\ldots$ & $(9.17 \pm 0.17) \times 10^{3}$ & $(3.29 \pm 0.05) \times 10^{3}$ & $(6.59 \pm 0.19) \times 10^{2}$ & $(9.04 \pm 1.60) \times 10^{1}$ \\
\hline & 1158 & $\ldots$ & $\ldots$ & $(6.83 \pm 0.10) \times 10^{3}$ & $(2.45 \pm 0.03) \times 10^{3}$ & $(4.97 \pm 0.14) \times 10^{2}$ & $(7.53 \pm 1.33) \times 10^{1}$ \\
\hline & 1505 & $\ldots$ & $\ldots$ & $(5.34 \pm 0.06) \times 10^{3}$ & $(1.91 \pm 0.02) \times 10^{3}$ & $(4.21 \pm 0.10) \times 10^{2}$ & $(7.10 \pm 1.01) \times 10^{1}$ \\
\hline & 1956 & $\ldots$ & $\ldots$ & $(4.24 \pm 0.04) \times 10^{3}$ & $(1.51 \pm 0.02) \times 10^{3}$ & $(3.29 \pm 0.07) \times 10^{2}$ & $(6.47 \pm 1.01) \times 10^{1}$ \\
\hline & 2649 & $\ldots$ & $\ldots$ & $(3.42 \pm 0.04) \times 10^{3}$ & $\ldots$ & $\ldots$ & $\ldots$ \\
\hline \multirow[t]{10}{*}{$353 \mathrm{GHz}$} & 53 & $\ldots$ & $\ldots$ & $\ldots$ & $(2.53 \pm 1.12) \times 10^{4}$ & $(5.52 \pm 2.45) \times 10^{3}$ & $(7.70 \pm 10.8) \times 10^{2}$ \\
\hline & 114 & $\ldots$ & $\ldots$ & $\ldots$ & $(1.43 \pm 0.19) \times 10^{4}$ & $(3.23 \pm 0.45) \times 10^{3}$ & $(4.65 \pm 1.72) \times 10^{2}$ \\
\hline & 187 & $\ldots$ & $\ldots$ & $\ldots$ & $(7.69 \pm 0.48) \times 10^{3}$ & $(1.66 \pm 0.11) \times 10^{3}$ & $(2.34 \pm 0.56) \times 10^{2}$ \\
\hline & 320 & $\ldots$ & $\ldots$ & $\ldots$ & $(4.23 \pm 0.12) \times 10^{3}$ & $(9.59 \pm 0.32) \times 10^{2}$ & $(1.51 \pm 0.18) \times 10^{2}$ \\
\hline & 502 & $\ldots$ & $\ldots$ & $\ldots$ & $(2.54 \pm 0.05) \times 10^{3}$ & $(5.91 \pm 0.16) \times 10^{2}$ & $(1.01 \pm 0.11) \times 10^{2}$ \\
\hline & 684 & $\ldots$ & $\ldots$ & $\ldots$ & $(1.70 \pm 0.03) \times 10^{3}$ & $(3.76 \pm 0.11) \times 10^{2}$ & $(6.22 \pm 0.82) \times 10^{1}$ \\
\hline & 890 & $\ldots$ & $\ldots$ & $\ldots$ & $(1.25 \pm 0.02) \times 10^{3}$ & $(2.72 \pm 0.08) \times 10^{2}$ & $(3.96 \pm 0.69) \times 10^{1}$ \\
\hline & 1158 & $\ldots$ & $\ldots$ & $\ldots$ & $(9.15 \pm 0.14) \times 10^{2}$ & $(1.87 \pm 0.09) \times 10^{2}$ & $(2.99 \pm 0.90) \times 10^{1}$ \\
\hline & 1505 & $\ldots$ & $\ldots$ & $\ldots$ & $(7.37 \pm 0.11) \times 10^{2}$ & $(1.54 \pm 0.09) \times 10^{2}$ & $(2.19 \pm 1.09) \times 10^{1}$ \\
\hline & 1956 & $\ldots$ & $\ldots$ & $\ldots$ & $(6.05 \pm 0.09) \times 10^{2}$ & $(1.34 \pm 0.04) \times 10^{2}$ & $(3.41 \pm 0.64) \times 10^{1}$ \\
\hline \multirow[t]{10}{*}{$217 \mathrm{GHz}$} & 53 & $\ldots$ & $\ldots$ & $\ldots$ & $\cdots$ & $(1.37 \pm 0.55) \times 10^{3}$ & $(2.16 \pm 2.41) \times 10^{2}$ \\
\hline & 114 & $\ldots$ & $\ldots$ & $\ldots$ & $\ldots$ & $(7.59 \pm 1.08) \times 10^{2}$ & $(1.10 \pm 0.42) \times 10^{2}$ \\
\hline & 187 & $\ldots$ & $\ldots$ & $\ldots$ & $\ldots$ & $(4.27 \pm 0.38) \times 10^{2}$ & $(7.80 \pm 1.60) \times 10^{1}$ \\
\hline & 320 & $\ldots$ & $\ldots$ & $\ldots$ & $\ldots$ & $(2.65 \pm 0.13) \times 10^{2}$ & $(5.68 \pm 0.60) \times 10^{1}$ \\
\hline & 502 & $\ldots$ & $\ldots$ & $\ldots$ & $\ldots$ & $(1.78 \pm 0.08) \times 10^{2}$ & $(4.77 \pm 0.47) \times 10^{1}$ \\
\hline & 684 & $\ldots$ & $\ldots$ & $\ldots$ & $\ldots$ & $(1.18 \pm 0.07) \times 10^{2}$ & $(3.05 \pm 0.43) \times 10^{1}$ \\
\hline & 890 & $\ldots$ & $\ldots$ & $\ldots$ & $\ldots$ & $(8.78 \pm 0.71) \times 10^{1}$ & $(1.87 \pm 0.48) \times 10^{1}$ \\
\hline & 1158 & $\ldots$ & $\ldots$ & $\ldots$ & $\ldots$ & $(5.97 \pm 1.57) \times 10^{1}$ & $(1.46 \pm 1.19) \times 10^{1}$ \\
\hline & 1505 & $\ldots$ & $\ldots$ & $\ldots$ & $\ldots$ & $(3.08 \pm 2.15) \times 10^{1}$ & $(8.40 \pm 16.4) \times 10^{0}$ \\
\hline & 1956 & $\ldots$ & $\ldots$ & $\ldots$ & $\ldots$ & $(4.73 \pm 0.65) \times 10^{1}$ & $(1.49 \pm 0.53) \times 10^{1}$ \\
\hline \multirow[t]{9}{*}{$143 \mathrm{GHz}$} & 53 & $\ldots$ & $\ldots$ & $\ldots$ & $\ldots$ & $\ldots$ & $(7.23 \pm 6.67) \times 10^{1}$ \\
\hline & 114 & $\ldots$ & $\ldots$ & $\ldots$ & $\ldots$ & $\ldots$ & $(3.05 \pm 1.43) \times 10^{1}$ \\
\hline & 187 & $\ldots$ & $\ldots$ & $\ldots$ & $\ldots$ & $\ldots$ & $(2.20 \pm 0.67) \times 10^{1}$ \\
\hline & 320 & $\ldots$ & $\ldots$ & $\ldots$ & $\ldots$ & $\ldots$ & $(2.14 \pm 0.31) \times 10^{1}$ \\
\hline & 502 & $\ldots$ & $\ldots$ & $\ldots$ & $\ldots$ & $\ldots$ & $(1.99 \pm 0.28) \times 10^{1}$ \\
\hline & 684 & $\ldots$ & $\ldots$ & $\ldots$ & $\ldots$ & $\ldots$ & $(1.55 \pm 0.29) \times 10^{1}$ \\
\hline & 890 & $\ldots$ & $\ldots$ & $\ldots$ & $\ldots$ & $\ldots$ & $(1.16 \pm 0.35) \times 10^{1}$ \\
\hline & 1158 & $\ldots$ & $\ldots$ & $\ldots$ & $\ldots$ & $\ldots$ & $(1.02 \pm 0.91) \times 10^{1}$ \\
\hline & 1505 & $\ldots$ & $\ldots$ & $\ldots$ & $\ldots$ & $\ldots$ & $(1.26 \pm 1.27) \times 10^{1}$ \\
\hline
\end{tabular}

Notes. Error bars here include uncertainties on foreground and CMB residuals, beam and projection. To obtain the CIB power spectra (given in Table D.2), the power spectra have to be further corrected for $C_{\mathrm{ClBcorr}}^{v \times v^{\prime}}$ (Eq. (4)), $C_{\mathrm{SZcorr}}^{v \times \nu^{\prime}}$ (Eq. (5)), and $C_{\mathrm{CIB}-\mathrm{SZcorr}}^{\nu \times v^{\prime}}$ (Eq. (9)). Moreover, the first two points (at $\ell=53$ and 114) have to be considered as upper limits (see Sect. 3.2.3). 
Planck Collaboration: Planck 2013 results. XXX.

Table D.2. Cosmic IR background power spectra.

\begin{tabular}{|c|c|c|c|c|c|c|c|}
\hline & \multirow[b]{2}{*}{$\ell_{\text {cent }}$} & \multicolumn{6}{|c|}{ Cross-power spectra, $C_{\ell}$, and statistical uncertainty $\left[\mathrm{Jy}^{2} \mathrm{sr}^{-1}\right]$} \\
\hline & & $3000 \mathrm{GHz}$ & $857 \mathrm{GHz}$ & $545 \mathrm{GHz}$ & $353 \mathrm{GHz}$ & $217 \mathrm{GHz}$ & $143 \mathrm{GHz}$ \\
\hline \multirow[t]{9}{*}{$3000 \mathrm{GHz}$} & 187 & $<5.6910^{5}$ & $(1.12 \pm 0.85) \times 10^{5}$ & $(3.94 \pm 3.88) \times 10^{4}$ & $(8.92 \pm 12.4) \times 10^{3}$ & $(5.59 \pm 26.7) \times 10^{2}$ & . \\
\hline & 320 & $(7.89 \pm 3.14) \times 10^{4}$ & $(7.49 \pm 2.09) \times 10^{4}$ & $(3.04 \pm 0.85) \times 10^{4}$ & $(1.13 \pm 0.27) \times 10^{4}$ & $(2.34 \pm 0.71) \times 10^{3}$ & . \\
\hline & 502 & $(3.46 \pm 1.15) \times 10^{4}$ & $(3.10 \pm 0.79) \times 10^{4}$ & $(1.23 \pm 0.33) \times 10^{4}$ & $(4.49 \pm 1.15) \times 10^{3}$ & $(1.18 \pm 0.34) \times 10^{3}$ & . \\
\hline & 684 & $(2.90 \pm 0.70) \times 10^{4}$ & $(2.24 \pm 0.47) \times 10^{4}$ & $(9.35 \pm 2.10) \times 10^{3}$ & $(2.87 \pm 0.73) \times 10^{3}$ & $(6.88 \pm 2.61) \times 10^{2}$ & . \\
\hline & 890 & $(1.87 \pm 0.47) \times 10^{4}$ & $(1.62 \pm 0.30) \times 10^{4}$ & $(6.61 \pm 1.34) \times 10^{3}$ & $(1.99 \pm 0.45) \times 10^{3}$ & $(2.34 \pm 1.66) \times 10^{2}$ & . \\
\hline & 1158 & $(1.43 \pm 0.40) \times 10^{4}$ & $(1.17 \pm 0.22) \times 10^{4}$ & $(4.71 \pm 0.93) \times 10^{3}$ & $(1.25 \pm 0.31) \times 10^{3}$ & $(3.55 \pm 1.43) \times 10^{2}$ & . \\
\hline & 1505 & $(1.09 \pm 0.41) \times 10^{4}$ & $(8.30 \pm 1.85) \times 10^{3}$ & $(3.12 \pm 0.76) \times 10^{3}$ & $(1.14 \pm 0.30) \times 10^{3}$ & $(2.41 \pm 1.07) \times 10^{2}$ & . \\
\hline & 1956 & $(1.05 \pm 0.58) \times 10^{4}$ & $(8.04 \pm 2.39) \times 10^{3}$ & $(3.16 \pm 1.00) \times 10^{3}$ & $(9.67 \pm 3.44) \times 10^{2}$ & $(2.58 \pm 1.14) \times 10^{2}$ & . \\
\hline & 2649 & $(1.06 \pm 1.04) \times 10^{4}$ & $(6.47 \pm 3.27) \times 10^{3}$ & $(2.61 \pm 1.38) \times 10^{3}$ & $\ldots$ & $\ldots$ & . \\
\hline \multirow[t]{11}{*}{$857 \mathrm{GHz}$} & 53 & $\ldots$ & $<2.7610^{6}$ & $<9.7310^{5}$ & $<2.9110^{5}$ & $<6.4310^{4}$ & $<1.8110^{4}$ \\
\hline & 114 & $\ldots$ & $<7.9910^{5}$ & $<3.2310^{5}$ & $<1.0510^{5}$ & $<2.4910^{4}$ & $<5.1210^{3}$ \\
\hline & 187 & $\ldots$ & $(2.87 \pm 0.37) \times 10^{5}$ & $(1.30 \pm 0.13) \times 10^{5}$ & $(4.30 \pm 0.41) \times 10^{4}$ & $(9.70 \pm 1.22) \times 10^{3}$ & $(1.84 \pm 0.45) \times 10^{3}$ \\
\hline & 320 & $\ldots$ & $(1.34 \pm 0.08) \times 10^{5}$ & $(6.36 \pm 0.30) \times 10^{4}$ & $(2.20 \pm 0.11) \times 10^{4}$ & $(5.26 \pm 0.53) \times 10^{3}$ & $(1.06 \pm 0.12) \times 10^{3}$ \\
\hline & 502 & $\ldots$ & $(7.20 \pm 0.26) \times 10^{4}$ & $(3.53 \pm 0.10) \times 10^{4}$ & $(1.25 \pm 0.06) \times 10^{4}$ & $(3.03 \pm 0.32) \times 10^{3}$ & $(6.52 \pm 0.59) \times 10^{2}$ \\
\hline & 684 & $\ldots$ & $(4.38 \pm 0.18) \times 10^{4}$ & $(2.21 \pm 0.07) \times 10^{4}$ & $(7.99 \pm 0.39) \times 10^{3}$ & $(1.88 \pm 0.22) \times 10^{3}$ & $(3.86 \pm 0.41) \times 10^{2}$ \\
\hline & 890 & $\ldots$ & $(3.23 \pm 0.09) \times 10^{4}$ & $(1.63 \pm 0.04) \times 10^{4}$ & $(5.88 \pm 0.27) \times 10^{3}$ & $(1.31 \pm 0.16) \times 10^{3}$ & $(2.55 \pm 0.30) \times 10^{2}$ \\
\hline & 1158 & $\ldots$ & $(2.40 \pm 0.05) \times 10^{4}$ & $(1.22 \pm 0.02) \times 10^{4}$ & $(4.25 \pm 0.17) \times 10^{3}$ & $(9.18 \pm 0.87) \times 10^{2}$ & $(1.76 \pm 0.23) \times 10^{2}$ \\
\hline & 1505 & $\ldots$ & $(1.83 \pm 0.03) \times 10^{4}$ & $(9.31 \pm 0.11) \times 10^{3}$ & $(3.24 \pm 0.10) \times 10^{3}$ & $(7.00 \pm 0.23) \times 10^{2}$ & $(1.23 \pm 0.16) \times 10^{2}$ \\
\hline & 1956 & $\ldots$ & $(1.46 \pm 0.02) \times 10^{4}$ & $(7.38 \pm 0.07) \times 10^{3}$ & $(2.54 \pm 0.07) \times 10^{3}$ & $(5.38 \pm 0.12) \times 10^{2}$ & $(1.03 \pm 0.15) \times 10^{2}$ \\
\hline & 2649 & $\ldots$ & $(1.16 \pm 0.01) \times 10^{4}$ & $(5.91 \pm 0.06) \times 10^{3}$ & $\ldots$ & $\ldots$ & $\ldots$ \\
\hline \multirow[t]{11}{*}{$545 \mathrm{GHz}$} & 53 & $\ldots$ & $\ldots$ & $<3.7410^{5}$ & $<1.1510^{5}$ & $<2.5810^{4}$ & $<7.0410^{3}$ \\
\hline & 114 & $\ldots$ & $\ldots$ & $<1.4510^{5}$ & $<4.8410^{4}$ & $<1.1610^{4}$ & $<2.5310^{3}$ \\
\hline & 187 & $\ldots$ & $\ldots$ & $(6.63 \pm 0.51) \times 10^{4}$ & $(2.22 \pm 0.16) \times 10^{4}$ & $(4.97 \pm 0.48) \times 10^{3}$ & $(1.01 \pm 0.19) \times 10^{3}$ \\
\hline & 320 & $\ldots$ & $\ldots$ & $(3.34 \pm 0.12) \times 10^{4}$ & $(1.19 \pm 0.05) \times 10^{4}$ & $(2.79 \pm 0.21) \times 10^{3}$ & $(5.98 \pm 0.67) \times 10^{2}$ \\
\hline & 502 & $\ldots$ & $\ldots$ & $(1.91 \pm 0.04) \times 10^{4}$ & $(6.93 \pm 0.23) \times 10^{3}$ & $(1.65 \pm 0.12) \times 10^{3}$ & $(3.77 \pm 0.39) \times 10^{2}$ \\
\hline & 684 & $\ldots$ & $\ldots$ & $(1.25 \pm 0.03) \times 10^{4}$ & $(4.61 \pm 0.16) \times 10^{3}$ & $(1.06 \pm 0.09) \times 10^{3}$ & $(2.29 \pm 0.27) \times 10^{2}$ \\
\hline & 890 & $\ldots$ & $\ldots$ & $(9.17 \pm 0.17) \times 10^{3}$ & $(3.39 \pm 0.11) \times 10^{3}$ & $(7.41 \pm 0.63) \times 10^{2}$ & $(1.54 \pm 0.19) \times 10^{2}$ \\
\hline & 1158 & $\ldots$ & $\ldots$ & $(6.83 \pm 0.10) \times 10^{3}$ & $(2.50 \pm 0.07) \times 10^{3}$ & $(5.38 \pm 0.35) \times 10^{2}$ & $(1.03 \pm 0.14) \times 10^{2}$ \\
\hline & 1505 & $\ldots$ & $\ldots$ & $(5.34 \pm 0.06) \times 10^{3}$ & $(1.93 \pm 0.04) \times 10^{3}$ & $(4.30 \pm 0.12) \times 10^{2}$ & $(7.09 \pm 1.80) \times 10^{1}$ \\
\hline & 1956 & $\ldots$ & $\ldots$ & $(4.24 \pm 0.04) \times 10^{3}$ & $(1.52 \pm 0.03) \times 10^{3}$ & $(3.30 \pm 0.07) \times 10^{2}$ & $(5.89 \pm 1.73) \times 10^{1}$ \\
\hline & 2649 & $\cdots$ & $\cdots$ & $(3.42 \pm 0.04) \times 10^{3}$ & $\ldots$ & $\ldots$ & $\ldots$ \\
\hline \multirow[t]{10}{*}{$353 \mathrm{GHz}$} & 53 & $\ldots$ & $\ldots$ & $\ldots$ & $<3.6810^{4}$ & $<8.0110^{3}$ & $<2.0510^{3}$ \\
\hline & 114 & $\ldots$ & $\ldots$ & $\ldots$ & $<1.6610^{4}$ & $<3.8210^{3}$ & $<8.2610^{2}$ \\
\hline & 187 & $\ldots$ & $\ldots$ & $\ldots$ & $(7.88 \pm 0.53) \times 10^{3}$ & $(1.75 \pm 0.15) \times 10^{3}$ & $(3.61 \pm 0.62) \times 10^{2}$ \\
\hline & 320 & $\ldots$ & $\ldots$ & $\ldots$ & $(4.35 \pm 0.18) \times 10^{3}$ & $(1.02 \pm 0.06) \times 10^{3}$ & $(2.32 \pm 0.24) \times 10^{2}$ \\
\hline & 502 & $\ldots$ & $\ldots$ & $\ldots$ & $(2.60 \pm 0.10) \times 10^{3}$ & $(6.21 \pm 0.38) \times 10^{2}$ & $(1.48 \pm 0.14) \times 10^{2}$ \\
\hline & 684 & $\ldots$ & $\ldots$ & $\ldots$ & $(1.74 \pm 0.07) \times 10^{3}$ & $(3.97 \pm 0.27) \times 10^{2}$ & $(9.42 \pm 1.06) \times 10^{1}$ \\
\hline & 890 & $\ldots$ & $\ldots$ & $\ldots$ & $(1.29 \pm 0.05) \times 10^{3}$ & $(2.87 \pm 0.20) \times 10^{2}$ & $(6.33 \pm 0.83) \times 10^{1}$ \\
\hline & 1158 & $\ldots$ & $\ldots$ & $\ldots$ & $(9.35 \pm 0.33) \times 10^{2}$ & $(1.99 \pm 0.14) \times 10^{2}$ & $(4.56 \pm 0.91) \times 10^{1}$ \\
\hline & 1505 & $\ldots$ & $\ldots$ & $\ldots$ & $(7.45 \pm 0.22) \times 10^{2}$ & $(1.59 \pm 0.10) \times 10^{2}$ & $(2.77 \pm 1.11) \times 10^{1}$ \\
\hline & 1956 & $\ldots$ & $\ldots$ & $\cdots$ & $(6.08 \pm 0.16) \times 10^{2}$ & $(1.35 \pm 0.05) \times 10^{2}$ & $(3.53 \pm 0.69) \times 10^{1}$ \\
\hline \multirow[t]{10}{*}{$217 \mathrm{GHz}$} & 53 & $\ldots$ & $\ldots$ & $\ldots$ & $\ldots$ & $<1.7810^{3}$ & $<4.7410^{2}$ \\
\hline & 114 & $\ldots$ & $\ldots$ & $\ldots$ & $\ldots$ & $<8.4710^{2}$ & $<1.8910^{2}$ \\
\hline & 187 & $\ldots$ & $\ldots$ & $\ldots$ & $\ldots$ & $(4.17 \pm 0.47) \times 10^{2}$ & $(1.04 \pm 0.19) \times 10^{2}$ \\
\hline & 320 & $\ldots$ & $\ldots$ & $\ldots$ & $\ldots$ & $(2.62 \pm 0.20) \times 10^{2}$ & $(7.49 \pm 0.81) \times 10^{1}$ \\
\hline & 502 & $\ldots$ & $\ldots$ & $\ldots$ & $\ldots$ & $(1.75 \pm 0.13) \times 10^{2}$ & $(5.87 \pm 0.58) \times 10^{1}$ \\
\hline & 684 & $\ldots$ & $\ldots$ & $\ldots$ & $\ldots$ & $(1.17 \pm 0.10) \times 10^{2}$ & $(3.93 \pm 0.50) \times 10^{1}$ \\
\hline & 890 & $\ldots$ & $\ldots$ & $\ldots$ & $\ldots$ & $(8.82 \pm 0.89) \times 10^{1}$ & $(2.64 \pm 0.52) \times 10^{1}$ \\
\hline & 1158 & $\ldots$ & $\ldots$ & $\ldots$ & $\ldots$ & $(6.42 \pm 1.61) \times 10^{1}$ & $(2.21 \pm 1.19) \times 10^{1}$ \\
\hline & 1505 & $\ldots$ & $\ldots$ & $\ldots$ & $\ldots$ & $(3.34 \pm 2.15) \times 10^{1}$ & $(1.07 \pm 1.65) \times 10^{1}$ \\
\hline & 1956 & $\cdots$ & $\cdots$ & $\cdots$ & $\cdots$ & $(4.74 \pm 0.65) \times 10^{1}$ & $(1.45 \pm 0.54) \times 10^{1}$ \\
\hline \multirow[t]{9}{*}{$143 \mathrm{GHz}$} & 53 & $\ldots$ & $\ldots$ & $\ldots$ & $\ldots$ & $\ldots$ & $<1.5510^{2}$ \\
\hline & 114 & $\ldots$ & $\ldots$ & $\ldots$ & $\ldots$ & $\ldots$ & $<6.4110^{1}$ \\
\hline & 187 & $\ldots$ & $\ldots$ & $\ldots$ & $\ldots$ & $\ldots$ & $(3.64 \pm 0.73) \times 10^{1}$ \\
\hline & 320 & $\ldots$ & $\ldots$ & $\ldots$ & $\ldots$ & $\ldots$ & $(3.23 \pm 0.35) \times 10^{1}$ \\
\hline & 502 & $\ldots$ & $\ldots$ & $\ldots$ & $\ldots$ & $\ldots$ & $(2.81 \pm 0.30) \times 10^{1}$ \\
\hline & 684 & $\ldots$ & $\ldots$ & $\ldots$ & $\ldots$ & $\ldots$ & $(2.27 \pm 0.29) \times 10^{1}$ \\
\hline & 890 & $\ldots$ & $\ldots$ & $\ldots$ & $\ldots$ & $\ldots$ & $(1.84 \pm 0.35) \times 10^{1}$ \\
\hline & 1158 & $\ldots$ & $\ldots$ & $\ldots$ & $\ldots$ & $\ldots$ & $(1.58 \pm 0.91) \times 10^{1}$ \\
\hline & 1505 & $\ldots$ & $\ldots$ & $\ldots$ & $\ldots$ & $\ldots$ & $(1.25 \pm 1.28) \times 10^{1}$ \\
\hline
\end{tabular}

Notes. These estimates are obtained from the CMB- and dust-free map power spectra (Table D.1), which have been corrected for SZ contaminations (Eqs. (5) and (9)), and for the spurious CIB contamination induced by our CMB template (Eq. (4)), computed using our best-fit model described in Sect. 5.5). The errors contain all the terms: statistical uncertainty; beam uncertainty; and errors from the SZ correction. Rows composed of entirely empty values have been omitted. 
Table D.3. Measured bispectrum at 217, 353, and $545 \mathrm{GHz}$ in $\mathrm{Jy}^{3} \mathrm{sr}^{-1}$ (see Sect. 4.3).

\begin{tabular}{|c|c|c|c|c|c|}
\hline \multicolumn{3}{|c|}{ Multipole bins } & \multicolumn{3}{|c|}{ Bispectrum coefficients $b_{\ell_{1} \ell_{2} \ell_{3}}$} \\
\hline$\ell_{1}$ & $\ell_{2}$ & $\ell_{3}$ & $217 \mathrm{GHz}$ & $353 \mathrm{GHz}$ & $545 \mathrm{GHz}$ \\
\hline 192 & 192 & 192 & $9.10 \times 10^{1}$ & $1.79 \times 10^{3}$ & $3.65 \times 10^{4}$ \\
\hline 192 & 192 & 320 & $3.35 \times 10^{1}$ & $7.84 \times 10^{2}$ & $2.81 \times 10^{4}$ \\
\hline 192 & 320 & 320 & $3.54 \times 10^{1}$ & $1.23 \times 10^{3}$ & $3.04 \times 10^{4}$ \\
\hline 192 & 320 & 448 & $1.71 \times 10^{1}$ & $6.90 \times 10^{2}$ & $1.45 \times 10^{4}$ \\
\hline 192 & 448 & 448 & $1.54 \times 10^{1}$ & $4.19 \times 10^{2}$ & $1.08 \times 10^{4}$ \\
\hline 192 & 448 & 576 & $1.13 \times 10^{1}$ & $2.81 \times 10^{2}$ & $8.16 \times 10^{3}$ \\
\hline 192 & 576 & 576 & $1.80 \times 10^{1}$ & $4.12 \times 10^{2}$ & $6.20 \times 10^{3}$ \\
\hline 192 & 576 & 704 & 3.92 & $1.59 \times 10^{2}$ & $6.15 \times 10^{3}$ \\
\hline 192 & 704 & 704 & 1.46 & $10^{1}$ & $<10^{3}$ \\
\hline 192 & 704 & 832 & 6.89 & $1.85 \times 10^{2}$ & $7.09 \times 10^{3}$ \\
\hline 192 & 832 & 832 & 3.89 & $1.70 \times 10^{2}$ & $6.69 \times 10^{3}$ \\
\hline 320 & 320 & 320 & $1.17 \times 10^{1}$ & $3.58 \times 10^{2}$ & $1.21 \times 10^{4}$ \\
\hline 320 & 320 & 448 & $1.06 \times 10^{1}$ & $4.25 \times 10^{2}$ & $9.13 \times 10^{3}$ \\
\hline 320 & 320 & 576 & 9.26 & $3.80 \times 10^{2}$ & $7.18 \times 10^{3}$ \\
\hline 320 & 448 & 448 & 9.83 & $2.29 \times 10^{2}$ & $6.82 \times 10^{3}$ \\
\hline 320 & 448 & 576 & 6.7 & $1.82 \times 10^{2}$ & $4.19 \times 10^{3}$ \\
\hline 320 & 448 & 704 & 6. & $3.02 \times 10^{2}$ & $6.53 \times 10^{3}$ \\
\hline 320 & 576 & 576 & & $1.80 \times 10^{2}$ & $2.68 \times 10^{3}$ \\
\hline 320 & 576 & 704 & & $7.60 \times 10^{2}$ & $2.40 \times 10^{3}$ \\
\hline 320 & 576 & 832 & & $1.31 \times 10^{2}$ & $3.14 \times 10^{3}$ \\
\hline 320 & 704 & 704 & & $1.40 \times 10^{2}$ & $5.82 \times 10^{3}$ \\
\hline 320 & 704 & 832 & & $10^{2}$ & $3.83 \times 10^{3}$ \\
\hline 320 & 832 & 832 & & $10^{2}$ & $5.06 \times 10^{3}$ \\
\hline 448 & 448 & 448 & & $10^{2}$ & $4.52 \times 10^{3}$ \\
\hline 448 & 448 & 576 & & 4.8 & $10^{3}$ \\
\hline 448 & 448 & 704 & 7 & $2.04 \times 10^{2}$ & $3.52 \times 10^{3}$ \\
\hline 448 & 448 & 832 & 2. & $1.17 \times 10^{2}$ & $2.91 \times 10^{3}$ \\
\hline 448 & 576 & 576 & 5 & $1.62 \times 10^{2}$ & $3.77 \times 10^{3}$ \\
\hline 448 & 576 & 704 & 7 & $9.08 \times 10^{2}$ & $1.93 \times 10^{3}$ \\
\hline 448 & 576 & 832 & 3 & $9.56 \times 10^{2}$ & $1.94 \times 10^{3}$ \\
\hline 448 & 704 & 704 & 7.2 & $1.49 \times 10^{2}$ & $3.28 \times 10^{3}$ \\
\hline 448 & 704 & 832 & 2.2 & $8.32 \times 10^{1}$ & $2.01 \times 10^{3}$ \\
\hline 448 & 832 & 832 & 2.70 & $1.04 \times 10^{2}$ & $2.75 \times 10^{3}$ \\
\hline 576 & 576 & 576 & 3.03 & $1.30 \times 10^{2}$ & $2.84 \times 10^{3}$ \\
\hline 576 & 576 & 704 & 4.62 & $8.39 \times 10^{1}$ & $1.41 \times 10^{3}$ \\
\hline 576 & 576 & 832 & 4.75 & $8.79 \times 10^{1}$ & $1.67 \times 10^{3}$ \\
\hline 576 & 704 & 704 & 2.82 & $7.55 \times 10^{1}$ & $\ldots$ \\
\hline 576 & 704 & 832 & 3.98 & $4.54 \times 10^{1}$ & \\
\hline 576 & 832 & 832 & & $5.93 \times 10^{1}$ & \\
\hline 704 & 704 & 704 & & $1.04 \times 10^{2}$ & \\
\hline
\end{tabular}

Notes. The covariance matrix is available at ESAs Planck Legacy Archive (PLA).

\section{References}

Addison, G. E., Dunkley, J., \& Spergel, D. N. 2012, MNRAS, 427, 1741 Addison, G. E., Dunkley, J., \& Bond, J. R. 2013, MNRAS, 436, 1896 Amblard, A., \& Cooray, A. 2007, ApJ, 670, 903

Amblard, A., Cooray, A., Serra, P., et al. 2010, A\&A, 518, L9

Amblard, A., Cooray, A., Serra, P., et al. 2011, Nature, 470, 510 Arendt, R. G., Odegard, N., Weiland, J. L., et al. 1998, ApJ, 508, 74 Behroozi, P. S., Wechsler, R. H., \& Conroy, C. 2013a, ApJ, 762, L31 Behroozi, P. S., Wechsler, R. H., \& Conroy, C. 2013b, ApJ, 770, 57 Benson, A. J., Bower, R. G., Frenk, C. S., et al. 2003, ApJ, 599, 38 Berta, S., Magnelli, B., Nordon, R., et al. 2011, A\&A, 532, A49

Béthermin, M., Dole, H., Lagache, G., Le Borgne, D., \& Penin, A. 2011, A\&A, $529, \mathrm{~A} 4$

Béthermin, M., Daddi, E., Magdis, G., et al. 2012a, ApJ, 757, L23 Béthermin, M., Doré, O., \& Lagache, G. 2012b, A\&A, 537, L5 Béthermin, M., Le Floc'h, E., Ilbert, O., et al. 2012c, A\&A, 542, A58 Béthermin, M., Wang, L., Doré, O., et al. 2013, A\&A, 557, A66 Blain, A. W. 1999, MNRAS, 309, 955

Blain, A. W., Ivison, R. J., \& Smail, I. 1998, MNRAS, 296, L29

Blain, A. W., Barnard, V. E., \& Chapman, S. C. 2003, MNRAS, 338, 733 Blain, A. W., Chapman, S. C., Smail, I., \& Ivison, R. 2004, ApJ, 611, 725 Boothroyd, A. I., Blagrave, K., Lockman, F. J., et al. 2011, A\&A, 536, A81 Boselli, A., Ciesla, L., Cortese, L., et al. 2012, A\&A, 540, A54
Boulanger, F., Abergel, A., Bernard, J. P., et al. 1996, A\&A, 312, 256 Bouwens, R. J., Illingworth, G. D., Franx, M., \& Ford, H. 2007, ApJ, 670, 928 Bouwens, R. J., Illingworth, G. D., Franx, M., et al. 2009, ApJ, 705, 936 Bouwens, R. J., Illingworth, G. D., Oesch, P. A., et al. 2012, ApJ, 754, 83 Burgarella, D., Buat, V., Gruppioni, C., et al. 2013, A\&A, 554, A70

Castellano, M., Fontana, A., Grazian, A., et al. 2012, A\&A, 540, A39

Chapin, E. L., Chapman, S. C., Coppin, K. E., et al. 2011, MNRAS, 411, 505 Chapman, S. C., Blain, A. W., Smail, I., \& Ivison, R. J. 2005, ApJ, 622, 772

Coles, P. 1993, MNRAS, 262, 1065

Cooray, A. 2006, MNRAS, 365,842

Cooray, A., \& Milosavljević, M. 2005, ApJ, 627, L89

Cooray, A., \& Sheth, R. 2002, Phys. Rep., 372, 1

Cooray, A., Amblard, A., Wang, L., et al. 2010, A\&A, 518, L22

Coppin, K., Halpern, M., Scott, D., et al. 2008, MNRAS, 384, 1597

Crawford, T. M., Schaffer, K. K., Bhattacharya, S., et al. 2014, ApJ, 784, 143

Croton, D. J., Springel, V., White, S. D. M., et al. 2006, MNRAS, 365, 11

Cucciati, O., Tresse, L., Ilbert, O., et al. 2012, A\&A, 539, A31

De Bernardis, F., \& Cooray, A. 2012, ApJ, 760, 14

De Lucia, G., \& Blaizot, J. 2007, MNRAS, 375, 2

Dole, H., Rieke, G. H., Lagache, G., et al. 2004, ApJS, 154, 93

Dole, H., Lagache, G., Puget, J. L., et al. 2006, A\&A, 451, 417

Draine, B. T., \& Lee, H. M. 1984, ApJ, 285, 89

Dunkley, J., Calabrese, E., Sievers, J., et al. 2013, J. Cosmol. Astropart. Phys., 7,25 
Dunne, L., Eales, S., Edmunds, M., et al. 2000, MNRAS, 315, 115

Fernandez-Conde, N., Lagache, G., Puget, J. L., \& Dole, H. 2008, A\&A, 481, 885

Gispert, R., Lagache, G., \& Puget, J. L. 2000, A\&A, 360, 1

Glenn, J., Conley, A., Béthermin, M., et al. 2010, MNRAS, 409, 109

Górski, K. M., Hivon, E., Banday, A. J., et al. 2005, ApJ, 622, 759

Gruppioni, C., Pozzi, F., Rodighiero, G., et al. 2013, MNRAS, 432, 23

Hajian, A., Viero, M. P., Addison, G., et al. 2012, ApJ, 744, 40

Hall, N. R., Keisler, R., Knox, L., et al. 2010, ApJ, 718, 632

Hildebrandt, H., van Waerbeke, L., Scott, D., et al. 2013, MNRAS, 429, 3230

Hopkins, A. M., \& Beacom, J. F. 2006, ApJ, 651, 142

Hwang, H. S., Elbaz, D., Magdis, G., et al. 2010, MNRAS, 409, 75

Kaiser, N. 1986, MNRAS, 222, 323

Kalberla, P. M. W., \& Dedes, L. 2008, A\&A, 487, 951

Kalberla, P. M. W., McClure-Griffiths, N. M., Pisano, D. J., et al. 2010, A\&A, 521, A17

Kauffmann, G., Colberg, J. M., Diaferio, A., \& White, S. D. M. 1999, MNRAS, 307,529

Kennicutt, R. C. 1998, ARA\&A, 36, 189

Kerp, J., Winkel, B., Ben Bekhti, N., Flöer, L., \& Kalberla, P. M. W. 2011, Astron. Nachr., 332, 637

Knox, L., Cooray, A., Eisenstein, D., \& Haiman, Z. 2001, ApJ, 550, 7

Lacasa, F., Aghanim, N., Kunz, M., \& Frommert, M. 2012, MNRAS, 421, 1982

Lacasa, F., Pénin, A., \& Aghanim, N. 2014, MNRAS, 439, 123

Lagache, G., Abergel, A., Boulanger, F., \& Puget, J. L. 1998, A\&A, 333, 709

Lagache, G., Abergel, A., Boulanger, F., Désert, F. X., \& Puget, J. L. 1999, A\&A, 344,322

Lagache, G., Haffner, L. M., Reynolds, R. J., \& Tufte, S. L. 2000, A\&A, 354, 247

Lagache, G., Dole, H., \& Puget, J. L. 2003, MNRAS, 338, 555

Lewis, A., \& Bridle, S. 2002, Phys. Rev. D, 66, 103511

López-Caniego, M., González-Nuevo, J., Massardi, M., et al. 2013, MNRAS, 430, 1566

Magdis, G. E., Daddi, E., Béthermin, M., et al. 2012, ApJ, 760, 6

Magnelli, B., Elbaz, D., Chary, R. R., et al. 2011, A\&A, 528, A35

Mandelbaum, R., Slosar, A., Baldauf, T., et al. 2013, MNRAS, 432, 1544

Mangum, J. G., Emerson, D. T., \& Greisen, E. W. 2007, A\&A, 474, 679

McClure-Griffiths, N. M., Pisano, D. J., Calabretta, M. R., et al. 2009, ApJS, 181, 398

Meny, C., Gromov, V., Boudet, N., et al. 2007, A\&A, 468, 171

Miville-Deschênes, M.-A., \& Lagache, G. 2005, ApJS, 157, 302

Miville-Deschênes, M.-A., Lagache, G., \& Puget, J.-L. 2002, A\&A, 393, 749

Miville-Deschênes, M.-A., Lagache, G., Boulanger, F., \& Puget, J.-L. 2007, A\&A, 469, 595

Miville-Deschênes, M.-A., Martin, P. G., Abergel, A., et al. 2010, A\&A, 518, L104

Mo, H. J., \& White, S. D. M. 1996, MNRAS, 282, 347

Moshir, M. E. A. 1992, version 2, JPL D

Moster, B. P., Somerville, R. S., Maulbetsch, C., et al. 2010, ApJ, 710, 903

Navarro, J. F., Frenk, C. S., \& White, S. D. M. 1997, ApJ, 490, 493

Neistein, E., Weinmann, S. M., Li, C., \& Boylan-Kolchin, M. 2011, MNRAS, 414,1405

Nguyen, H. T., Schulz, B., Levenson, L., et al. 2010, A\&A, 518, L5

Nidever, D. L., Majewski, S. R., \& Burton, W. B. 2008, ApJ, 679, 432

Oesch, P. A., Bouwens, R. J., Illingworth, G. D., et al. 2012a, ApJ, 759, 135

Oesch, P. A., Bouwens, R. J., Illingworth, G. D., et al. 2012b, ApJ, 745, 110

Oliver, S., Frost, M., Farrah, D., et al. 2010, MNRAS, 405, 2279

Paradis, D., Veneziani, M., Noriega-Crespo, A., et al. 2010, A\&A, 520, L8

Pascale, E., Ade, P. A. R., Bock, J. J., et al. 2009, ApJ, 707, 1740

Pénin, A., Doré, O., Lagache, G., \& Béthermin, M. 2012a, A\&A, 537, A137

Pénin, A., Lagache, G., Noriega-Crespo, A., et al. 2012b, A\&A, 543, A123

Pénin, A., Lacasa, F., \& Aghanim, N. 2014, MNRAS, 439, 143

Planck Collaboration XVIII. 2011, A\&A, 536, A18

Planck Collaboration XIX. 2011, A\&A, 536, A19

Planck Collaboration XXIV. 2011, A\&A, 536, A24

Planck Collaboration Int. VII. 2013, A\&A, 550, A133

Planck Collaboration 2013, The Explanatory Supplement to the Planck 2013 results, http://www.sciops.esa.int/wikiSI/planckpla/index. php?title=Main_Page (ESA)

Planck Collaboration I. 2014, A\&A, 571, A1

Planck Collaboration II. 2014, A\&A, 571, A2

Planck Collaboration III. 2014, A\&A, 571, A3

Planck Collaboration IV. 2014, A\&A, 571, A4

Planck Collaboration V. 2014, A\&A, 571, A5

Planck Collaboration VI. 2014, A\&A, 571, A6

Planck Collaboration VII. 2014, A\&A, 571, A7

Planck Collaboration VIII. 2014, A\&A, 571, A8

Planck Collaboration IX. 2014, A\&A, 571, A9
Planck Collaboration X. 2014, A\&A, 571, A10

Planck Collaboration XI. 2014, A\&A, 571, A11

Planck Collaboration XII. 2014, A\&A, 571, A12

Planck Collaboration XIII. 2014, A\&A, 571, A13

Planck Collaboration XIV. 2014, A\&A, 571, A14

Planck Collaboration XV. 2014, A\&A, 571, A15

Planck Collaboration XVI. 2014, A\&A, 571, A16

Planck Collaboration XVII. 2014, A\&A, 571, A17

Planck Collaboration XVIII. 2014, A\&A, 571, A18

Planck Collaboration XIX. 2014, A\&A, 571, A19

Planck Collaboration XX. 2014, A\&A, 571, A20

Planck Collaboration XXI. 2014, A\&A, 571, A21

Planck Collaboration XXII. 2014, A\&A, 571, A22

Planck Collaboration XXIII. 2014, A\&A, 571, A23

Planck Collaboration XXIV. 2014, A\&A,, 571, A24

Planck Collaboration XXV. 2014, A\&A, 571, A25

Planck Collaboration XXVI. 2014, A\&A, 571, A26

Planck Collaboration XXVII. 2014, A\&A, 571, A27

Planck Collaboration XXVIII. 2014, A\&A, 571, A28

Planck Collaboration XXIX. 2014, A\&A, 571, A29

Planck Collaboration XXX. 2014, A\&A, 571, A30

Planck Collaboration XXXI. 2014, A\&A, 571, A31

Ponthieu, N., Grain, J., \& Lagache, G. 2011, A\&A, 535, A90

Reach, W. T., Wall, W. F., \& Odegard, N. 1998, ApJ, 507, 507

Reddy, N. A., \& Steidel, C. C. 2009, ApJ, 692, 778

Reichardt, C. L., Shaw, L., Zahn, O., et al. 2012, ApJ, 755, 70

Riechers, D. A., Bradford, C. M., Clements, D. L., et al. 2013, Nature, 496, 329

Rodighiero, G., Vaccari, M., Franceschini, A., et al. 2010, A\&A, 515, A8

Rodríguez-Puebla, A., Drory, N., \& Avila-Reese, V. 2012, ApJ, 756, 2

Rodríguez-Puebla, A., Avila-Reese, V., \& Drory, N. 2013, ApJ, 767, 92

Saunders, W., Rowan-Robinson, M., \& Lawrence, A. 1992, MNRAS, 258, 134

Sehgal, N., Bode, P., Das, S., et al. 2010, ApJ, 709, 920

Shang, C., Haiman, Z., Knox, L., \& Oh, S. P. 2012, MNRAS, 421, 2832

Spergel, D., \& Goldberg, D. 1999, Phys. Rev. D, 59, 1

Steidel, C. C., Adelberger, K. L., Dickinson, M., et al. 1998, ApJ, 492, 428

Tegmark, M., \& Peebles, P. J. E. 1998, ApJ, 500, L79

Tinker, J., Kravtsov, A. V., Klypin, A., et al. 2008, ApJ, 688, 709

Tinker, J. L., Robertson, B. E., Kravtsov, A. V., et al. 2010, ApJ, 724, 878

Tristram, M., Macías-Pérez, J. F., Renault, C., \& Santos, D. 2005, MNRAS, 358, 833

Tucci, M., Toffolatti, L., de Zotti, G., \& Martínez-González, E. 2011, A\&A, 533, A57

Vaccari, M., Marchetti, L., Franceschini, A., et al. 2010, A\&A, 518, L20

Venzmer, M. S., Kerp, J., \& Kalberla, P. M. W. 2012, A\&A, 547, A12

Verde, L., Heavens, A. F., Percival, W. J., et al. 2002, MNRAS, 335, 432

Viero, M. P., Ade, P. A. R., Bock, J. J., et al. 2009, ApJ, 707, 1766

Viero, M. P., Moncelsi, L., Quadri, R. F., et al. 2013a, ApJ, 779, 32

Viero, M. P., Wang, L., Zemcov, M., et al. 2013b, ApJ, 772, 77

Wang, L., Farrah, D., Oliver, S. J., et al. 2013, MNRAS, 431, 648

Weaver, H. 1974, Highlights of Astronomy, 3, 423

Wechsler, R. H., Gross, M. A. K., Primack, J. R., Blumenthal, G. R., \& Dekel, A. 1998, ApJ, 506, 19

Weinmann, S. M., Neistein, E., \& Dekel, A. 2011, MNRAS, 417, 2737

Winkel, B., Kalberla, P. M. W., Kerp, J., \& Flöer, L. 2010, ApJS, 188, 488

Xia, J.-Q., Negrello, M., Lapi, A., et al. 2012, MNRAS, 422, 1324

Yang, X., Mo, H. J., \& van den Bosch, F. C. 2003, MNRAS, 339, 1057

Yang, X., Mo, H. J., Jing, Y. P., \& van den Bosch, F. C. 2005, MNRAS, 358, 217

Zemcov, M., Blain, A., Halpern, M., \& Levenson, L. 2010, ApJ, 721, 424

1 APC, AstroParticule et Cosmologie, Université Paris Diderot, CNRS/IN2P3, CEA/lrfu, Observatoire de Paris, Sorbonne Paris Cité, 10 rue Alice Domon et Léonie Duquet, 75205 Paris Cedex 13, France

2 Aalto University Metsähovi Radio Observatory, Metsähovintie 114, 02540 Kylmälä, Finland

3 African Institute for Mathematical Sciences, 6-8 Melrose Road, 7945 Muizenberg, Cape Town, South Africa

4 Agenzia Spaziale Italiana Science Data Center, via del Politecnico snc, 00133 Roma, Italy

5 Agenzia Spaziale Italiana, via le Liegi 26, Roma, Italy

6 Argelander-Institut für Astronomie, Universität Bonn, Auf dem Hügel 71, 53121 Bonn, Germany

7 Astrophysics Group, Cavendish Laboratory, University of Cambridge, J J Thomson Avenue, Cambridge CB3 OHE, UK 
8 Astrophysics \& Cosmology Research Unit, School of Mathematics, Statistics \& Computer Science, University of KwaZulu-Natal, Westville Campus, Private Bag X54001, 4000 Durban, South Africa

9 Atacama Large Millimeter/submillimeter Array, ALMA Santiago Central Offices, Alonso de Cordova 3107, Vitacura, Casilla 763 0355 Santiago, Chile

10 CITA, University of Toronto, 60 St. George St., Toronto ON M5S 3H8, Canada

11 CNRS, IRAP, 9 Av. colonel Roche, BP 44346, 31028 Toulouse Cedex 4, France

12 California Institute of Technology, Pasadena, California, USA

13 Centre for Theoretical Cosmology, DAMTP, University of Cambridge, Wilberforce Road, Cambridge CB3 0WA, UK

14 Centro de Estudios de Física del Cosmos de Aragón (CEFCA), Plaza San Juan 1, planta 2, 44001 Teruel, Spain

15 Computational Cosmology Center, Lawrence Berkeley National Laboratory, Berkeley, California, USA

16 Consejo Superior de Investigaciones Científicas (CSIC), 28049 Madrid, Spain

17 DSM/Irfu/SPP, CEA-Saclay, 91191 Gif-sur-Yvette Cedex, France

18 DTU Space, National Space Institute, Technical University of Denmark, Elektrovej 327, 2800 Kgs. Lyngby, Denmark

19 Département de Physique Théorique, Université de Genève, 24 Quai E. Ansermet, 1211 Genève 4, Switzerland

20 Departamento de Física Fundamental, Facultad de Ciencias, Universidad de Salamanca, 37008 Salamanca, Spain

21 Departamento de Física, Universidad de Oviedo, Avda. Calvo Sotelo s/n, 33007 Oviedo, Spain

22 Department of Astronomy and Astrophysics, University of Toronto, 50 Saint George Street, Toronto, Ontario, Canada

23 Department of Astrophysics/IMAPP, Radboud University Nijmegen, PO Box 9010, 6500 GL Nijmegen, The Netherlands

24 Department of Electrical Engineering and Computer Sciences, University of California, Berkeley, California, USA

25 Department of Physics \& Astronomy, University of British Columbia, 6224 Agricultural Road, Vancouver, British Columbia, Canada

26 Department of Physics and Astronomy, Dana and David Dornsife College of Letter, Arts and Sciences, University of Southern California, Los Angeles CA 90089, USA

27 Department of Physics and Astronomy, University College London, London WC1E 6BT, UK

28 Department of Physics, Florida State University, Keen Physics Building, 77 Chieftan Way, Tallahassee, Florida, USA

29 Department of Physics, Gustaf Hällströmin katu 2a, University of Helsinki, 00014 Helsinki, Finland

30 Department of Physics, Princeton University, Princeton, New Jersey, USA

31 Department of Physics, University of California, Berkeley, California, USA

32 Department of Physics, University of California, One Shields Avenue, Davis, California, USA

33 Department of Physics, University of California, Santa Barbara, California, USA

34 Department of Physics, University of Illinois at Urbana-Champaign, 1110 West Green Street, Urbana, Illinois, USA

35 Dipartimento di Fisica e Astronomia G. Galilei, Università degli Studi di Padova, via Marzolo 8, 35131 Padova, Italy

36 Dipartimento di Fisica e Scienze della Terra, Università di Ferrara, via Saragat 1, 44122 Ferrara, Italy

37 Dipartimento di Fisica, Università La Sapienza, P.le A. Moro 2, 00135 Roma, Italy

38 Dipartimento di Fisica, Università degli Studi di Milano, via Celoria, 16, 20133 Milano, Italy

39 Dipartimento di Fisica, Università degli Studi di Trieste, via A. Valerio 2, 34127 Trieste, Italy

40 Dipartimento di Fisica, Università di Roma Tor Vergata, via della Ricerca Scientifica 1, 00133 Roma, Italy

41 Discovery Center, Niels Bohr Institute, Blegdamsvej 17, 2100 Copenhagen, Denmark
42 Dpto. Astrofísica, Universidad de La Laguna (ULL), $38206 \mathrm{La}$ Laguna, Tenerife, Spain

43 European Southern Observatory, ESO Vitacura, Alonso de Cordova 3107, Vitacura, Casilla 19001 Santiago, Chile

44 European Space Agency, ESAC, Planck Science Office, Camino bajo del Castillo, s/n, Urbanización Villafranca del Castillo, Villanueva de la Cañada, Madrid, Spain

45 European Space Agency, ESTEC, Keplerlaan 1, 2201 AZ Noordwijk, The Netherlands

46 Finnish Centre for Astronomy with ESO (FINCA), University of Turku, Väisäläntie 20, 21500 Piikkiö, Finland

47 Haverford College Astronomy Department, 370 Lancaster Avenue, Haverford, Pennsylvania, USA

48 Helsinki Institute of Physics, Gustaf Hällströmin katu 2, University of Helsinki, 00014 Helsinki, Finland

49 INAF - Osservatorio Astronomico di Padova, Vicolo dell'Osservatorio 5, 35122 Padova, Italy

50 INAF - Osservatorio Astronomico di Roma, via di Frascati 33, 00040 Monte Porzio Catone, Italy

51 INAF - Osservatorio Astronomico di Trieste, via G.B. Tiepolo 11, 34143 Trieste, Italy

52 INAF Istituto di Radioastronomia, via P. Gobetti 101, 40129 Bologna, Italy

53 INAF/IASF Bologna, via Gobetti 101, 40129 Bologna, Italy

54 INAF/IASF Milano, via E. Bassini 15, 20133 Milano, Italy

55 INFN, Sezione di Bologna, via Irnerio 46, 40126 Bologna, Italy

56 INFN, Sezione di Roma 1, Università di Roma Sapienza, Piazzale Aldo Moro 2, 00185 Roma, Italy

57 IPAG: Institut de Planétologie et d'Astrophysique de Grenoble, Université Joseph Fourier, Grenoble 1 / CNRS-INSU, UMR 5274, 38041 Grenoble, France

58 ISDC Data Centre for Astrophysics, University of Geneva, Ch. d'Ecogia 16, 1290 Versoix, Switzerland

59 IUCAA, Post Bag 4, Ganeshkhind, Pune University Campus, 411007 Pune, India

${ }^{60}$ Imperial College London, Astrophysics group, Blackett Laboratory, Prince Consort Road, London SW7 2AZ, UK

61 Infrared Processing and Analysis Center, California Institute of Technology, Pasadena CA 91125, USA

62 Institut Néel, CNRS, Université Joseph Fourier Grenoble I, 25 rue des Martyrs, Grenoble, France

63 Institut Universitaire de France, 103 bd Saint-Michel, 75005 Paris, France

64 Institut d'Astrophysique Spatiale, CNRS (UMR8617) Université Paris-Sud 11, Bâtiment 121, 91405 Orsay, France

65 Institut d'Astrophysique de Paris, CNRS (UMR7095), 98bis Bd Arago, 75014 Paris, France

${ }^{66}$ Institute for Space Sciences, 077125 Bucharest-Magurale, Romania

67 Institute of Astronomy and Astrophysics, Academia Sinica, 10617 Taipei, Taiwan

68 Institute of Astronomy, University of Cambridge, Madingley Road, Cambridge CB3 0HA, UK

69 Institute of Theoretical Astrophysics, University of Oslo, Blindern, 0315 Oslo, Norway

70 Instituto de Astrofísica de Canarias, C/Vía Láctea s/n, 38205 La Laguna, Tenerife, Spain

71 Instituto de Física de Cantabria (CSIC-Universidad de Cantabria), Avda. de los Castros s/n, 39005 Santander, Spain

72 Jet Propulsion Laboratory, California Institute of Technology, 4800 Oak Grove Drive, Pasadena, California, USA

73 Jodrell Bank Centre for Astrophysics, Alan Turing Building, School of Physics and Astronomy, The University of Manchester, Oxford Road, Manchester M13 9PL, UK

74 Kavli Institute for Cosmology Cambridge, Madingley Road, Cambridge CB3 0HA, UK

75 LAL, Université Paris-Sud, CNRS/IN2P3, 91898 Orsay, France

${ }^{76}$ LERMA, CNRS, Observatoire de Paris, 61 avenue de l'Observatoire, 75014 Paris, France 
77 Laboratoire AIM, IRFU/Service d'Astrophysique - CEA/DSM CNRS - Université Paris Diderot, Bât. 709, CEA-Saclay, 91191 Gif-sur-Yvette Cedex, France

78 Laboratoire Traitement et Communication de l'Information, CNRS (UMR 5141) and Télécom ParisTech, 46 rue Barrault, 75634 Paris Cedex 13, France

79 Laboratoire de Physique Subatomique et de Cosmologie, Université Joseph Fourier Grenoble I, CNRS/IN2P3, Institut National Polytechnique de Grenoble, 53 rue des Martyrs, 38026 Grenoble Cedex, France

${ }^{80}$ Laboratoire de Physique Théorique, Université Paris-Sud 11 \& CNRS, Bâtiment 210, 91405 Orsay, France

81 Lawrence Berkeley National Laboratory, Berkeley, California, USA

82 Max-Planck-Institut für Astrophysik, Karl-Schwarzschild-Str. 1, 85741 Garching, Germany

83 McGill Physics, Ernest Rutherford Physics Building, McGill University, 3600 rue University, Montréal, QC H3A 2T8, Canada

${ }^{84}$ MilliLab, VTT Technical Research Centre of Finland, Tietotie 3, 02044 Espoo, Finland

85 National University of Ireland, Department of Experimental Physics, Maynooth, Co. Kildare, Ireland

86 Niels Bohr Institute, Blegdamsvej 17, 2100 Copenhagen, Denmark

87 Observational Cosmology, Mail Stop 367-17, California Institute of Technology, Pasadena CA 91125, USA

88 Optical Science Laboratory, University College London, Gower Street, London, UK
89 SB-ITP-LPPC, EPFL, 1015 Lausanne, Switzerland

90 SISSA, Astrophysics Sector, via Bonomea 265, 34136 Trieste, Italy

91 School of Physics and Astronomy, Cardiff University, Queens Buildings, The Parade, Cardiff CF24 3AA, UK

92 Space Research Institute (IKI), Russian Academy of Sciences, Profsoyuznaya Str, 84/32, 117997 Moscow, Russia

93 Space Sciences Laboratory, University of California, Berkeley, California, USA

94 Special Astrophysical Observatory, Russian Academy of Sciences, Nizhnij Arkhyz, Zelenchukskiy region, 369167 KarachaiCherkessian Republic, Russia

95 Stanford University, Dept of Physics, Varian Physics Bldg, 382 via Pueblo Mall, Stanford, California, USA

96 Sub-Department of Astrophysics, University of Oxford, Keble Road, Oxford OX1 3RH, UK

97 Theory Division, PH-TH, CERN, 1211 Geneva 23, Switzerland

98 UPMC Univ. Paris 06, UMR7095, 98 bis boulevard Arago, 75014 Paris, France

99 Université de Toulouse, UPS-OMP, IRAP, 31028 Toulouse Cedex 4, France

100 Universities Space Research Association, Stratospheric Observatory for Infrared Astronomy, MS 232-11, Moffett Field CA 94035, USA

101 University of Granada, Departamento de Física Teórica y del Cosmos, Facultad de Ciencias, 18071 Granada, Spain

102 Warsaw University Observatory, Aleje Ujazdowskie 4, 00-478 Warszawa, Poland 